Received Date: 30/10/2014

Accepted Date: 30/10/2014

Article Type: Original Article

\title{
GGR Biennial Critical Review: Analytical Developments Since 2012
}

Michael Wiedenbeck (1)*, L. Paul Bédard (2) Roxana Bugoi (3), Mary Horan (4), Kathryn Linge (5), Silke Merchel (6), Luiz F.G. Morales (1), Dany Savard (2), A. Kate Souders (7) and Paul Sylvester (8)

(1) Helmholtz Zentrum Potsdam, Deutsches GeoForschungsZentrum, Telegrafenberg, 14473 Potsdam, Germany

(2) Sciences de la Terre, Université du Québec à Chicoutimi, Chicoutimi, QC, Canada

(3) Roxana Bugoi, Horia Hulubei National Institute for Nuclear Physics and Engineering, P.O. Box MG-6, Bucharest-Măgurele, RO-077125, Romania

(4) Department of Terrestrial Magnetism, Carnegie Institution of Washington, 5241 Broad Branch Road NW, Washington DC, 20015, USA

(5) Curtin Water Quality Research Centre, Curtin University, GPO Box U1987 Perth, Western Australia, 6845, Australia

(6) Helmholtz-Zentrum Dresden-Rossendorf, Helmholtz Institute for Resource Technology, Bautzner Landstrasse 400, 01328 Dresden, Germany

(7) Center for Meteorite Studies, School of Earth and Space Exploration, Arizona State University, Tempe, Arizona, 85287-1404, USA

(8) Department of Geosciences, Texas Tech University, 125 Science Building, Lubbock, Texas, 79409-1053, USA

* Corresponding author. e-mail: michael.wiedenbeck@gfz-potsdam.de

Advances in the chemical, crystallographic and isotopic characterisation of geological and environmental materials can often be ascribed to technological improvements in analytical hardware or to innovative approaches to data acquisition and/or its interpretation. This biennial review addresses key laboratory methods that form much of the foundation for analytical geochemistry; again this contribution is presented as a compendium of laboratory techniques. We highlight advances that have appeared since January 2012 and that are of particular significance for the chemical and isotopic characterisation of geomaterials. Prominent scientists from the selected analytical fields present publications they judge to be particular noteworthy, providing background information about the method and assessing where further opportunities might be anticipated. In addition to well established technologies such as thermal ionisation mass spectrometry and plasma emission spectroscopy, this publication also presents new or rapidly growing methods such as electron backscattered diffraction analysis and atom probe tomography - a very sensitive method providing atomic scale information.

This article has been accepted for publication and undergone full peer review but has not been through the copyediting, typesetting, pagination and proofreading process, which may lead to differences between this version and the Version of Record. Please cite this article as doi: 10.1111/j.1751-908X.2014.00347.x

This article is protected by copyright. All rights reserved. 
Keywords: ICP-MS, laser ablation, mass spectrometry, ICP-AES, microwave plasma source, TIMS, isotopic analysis, geochronology, trace element analysis, calibration, XRF, environmental sampling, AMS, Ion Beam Analysis, radionuclides, SIMS, ion probe, microanalysis, FIB, EBSD, atom probe tomography.

\section{Received 23 Oct 14 - Accepted 30 Oct 14}

This contribution marks the continuation of Geostandards and Geoanalytical Research's biennial review series, which describes advances in both methods and instrumentation for the characterisation of geomaterials. Such reviews in Geostandards first appeared in 2006, where five individual contributions devoted to specific analytical methods were presented. The most recent contribution (Wiedenbeck et al. 2012) was presented as a single compilation, and this format has been continued here. One change from the review that appeared two years previously is that the segment devoted to reference materials in geoanalytical and environmental research has been removed, making this current contribution focussed entirely on laboratory instrumentation and innovative strategies that advance data quality. Specific chapters contained in this review, and their corresponding page numbers, are:

- Plasma source mass spectrometry and laser ablation sampling

page $\mathrm{xxx}$

- Plasma source emission spectroscopy

page $x x x$

- X-ray fluorescence methods

page $x x x$

- TIMS ion detection

page $x x x$

- Accelerator-based methods

page $x x x$

- Secondary ion mass spectrometry

page $x x x$

- Focused ion beam method

page $x x x$

- Electron backscatter diffraction

page $x x x$

- Atom probe tomography

page $\mathrm{xxx}$

\section{Advances in plasma source mass spectrometry and laser ablation sampling}

(Contribution by: P.J. Sylvester and A.K. Souders)

\section{Overview of ICP-MS and laser ablation ICP-MS}

Inductively coupled plasma source-mass spectrometry (ICP-MS) is used principally for rapid, precise and accurate trace element determination in solid, liquid and gas samples. It is also

This article is protected by copyright. All rights reserved. 
used increasingly for both isotopic and speciation determinations. The technique became commercially available in the mid-1980s, and since then has grown rapidly as a technique capable of element and isotopic measurements over a wide dynamic range (major to sub-trace contents) at a range of spatial resolutions (bulk rock to micrometre-size inclusions). Samples are introduced to the ICP-MS as solids dissolved in acidic solutions, sometimes purified to separate particular elements of analytical interest, or as fine aerosols produced by laser ablation. For solutions, a nebuliser converts the liquid into a fine spray of droplets, the largest of which are removed by a spray chamber, before the remaining aerosol is injected into the ICP where the atoms of the elements in the sample are converted into ions.

In general, a mass spectrometer has four main parts: the ion source, mass filter, detector, and vacuum system. ICP mass spectrometers come as single-collector (detector) instruments, which detect ions sequentially by mass, and multi-collector (detector) instruments capable of simultaneous detection of ions. Quadrupole and magnetic sector ICP-MS instruments are the two most common mass analysers used in the geosciences. A quadrupole mass analyser consists of four parallel rods with an AC or DC voltage applied to each set of rods so as to create an electrostatic field between the rods (Linge and Jarvis 2009). For given combination of AC and DC voltages, only ions of a certain mass-to-charge ratio will pass through the quadrupole; all other ions are filtered from their original path. A magnetic sector separates ions in a magnetic field as a function of their mass-to-charge ratio between poles of a magnet (Rehkämper et al. 2001).

Three different detector types are found in ICP-MS instruments. Discrete-dynode and continuous dynode detectors operate in both pulse-counting and analogue modes that have the advantage of both an instantaneous response time and a large dynamic range capable of detecting concentrations from sub-pg/g to high- $\mu \mathrm{g} / \mathrm{g}$. Faraday cups are the third type of detector in common use in ICP mass spectrometer, they have the ability to deal with excessively high count rates $\left(10^{9}\right.$ to $\left.10^{12} \mathrm{cps}\right)$, are effectively $100 \%$ efficient, and have stable response characteristics. Unfortunately response of the Faraday cups is not also instantaneous, meaning Faraday cup readouts lag behind that of electron multipliers, increasing analytical uncertainty when the two different detector types are used for the same measurement.

Laser ablation - ICP-MS (LA-ICP-MS) is an in situ analytical technique in which laser pulses are used to remove small volumes of a sample, and the resulting aerosol is introduced into the ICP-MS for analysis. In the geosciences this technique is used for determining the elemental and isotopic composition of micrometre-sized areas of minerals, volcanic glasses, metal alloys, fused rocks, fluid inclusions, experimental charges and variety of environmental

This article is protected by copyright. All rights reserved. 
proxies such as bones, teeth and calcareous shells (Sylvester 2008). Samples are typically prepared as a thin polished slice or slab, and are often imaged using light optical and/or scanning electron microscopy in advance so as to identify domains the best domains for laser sampling.

A laser ablation system is connected to an ICP-MS via an airtight sample cell, where the samples are ablated, and plastic tubing, which carries the ablated aerosols to the ICP in a helium + argon gas stream. As with solution nebulisation, the aerosols are ionised in the ICP torch, and then separated based on their mass to charge ratios by a mass spectrometer and measured with a detector. A quadrupole mass filter is the most common type of mass spectrometer used for LA-ICP-MS, but magnetic sector field mass analysers incorporating single- or multiple-collectors (MC) are increasingly employed because of their superior sensitivity and precision for isotope ratio measurements. The detector in single-collector instruments is typically a secondary electron multiplier, whereas multiple Faraday cups $( \pm$ ion counters) are used in MC devices.

Deep ultraviolet (UV) nanosecond pulsed lasers are most commonly used for LA-ICP-MS, either a neodymium-doped yttrium aluminium garnet (Nd:YAG) solid-state laser using the frequency quintupled $213 \mathrm{~nm}$ output, or an argon-fluoride (ArF) excimer gas laser using its fundamental $193 \mathrm{~nm}$ wavelength. UV light is absorbed more completely than infrared light by many geomaterials, particularly those that are transparent or poor in iron. There has been recent interest in using femtosecond pulsed lasers based on evidence that they produce aerosols with a large proportion of small particle sizes and negligible sample melting at the ablation site, improving analytical performance (Shaheen et al. 2012).

The moderate capital cost, robust design, multi-element capability, short analysis times, minimal sample preparation requirements, and good precision and accuracy of LA-ICP-MS compared with competing techniques for in situ micrometre-scale, chemical analysis has led to its widespread use in university, government and industry research laboratories worldwide.

Five notable areas of developments in plasma source mass spectrometry and laser sampling for geochemistry and geochronology since 2012 are presented in this contribution: (1) Sample digestion for solution ICP-MS analysis; (2) Performance of the new generation MCICP-MS instruments; (3) Improved spatial resolution of laser analysis; (4) Inter-laboratory comparison of in situ U-Pb zircon geochronology; and (5) New data processing software for LA-ICP-MS. Finally, we note that a recent thematic issue in Geostandards and Geoanalytical Research (Sylvester 2014 and associated contributions) gives a recent perspective on a variety of key aspects of laser-based sampling methods as applied to geomaterials.

This article is protected by copyright. All rights reserved. 


\section{Sample digestion for solution ICP-MS analysis}

ICP-MS is used routinely for multi-element and isotopic analysis of geological materials. LA-ICP-MS has rapidly become a popular choice for the in situ determination of elemental and isotopic compositions of individual minerals, while solution ICP-MS remains the preferred method of sample introduction for characterising the chemical compositions of whole rocks and high-precision isotopic analyses of geological materials. Sample digestion is a critical step when preparing geological samples for solution ICP-MS analysis; it is most often the limiting factor in obtaining rapid and accurate results. Techniques that have been developed include fusion and sintering procedures, open and closed vessel acid digestion, microwave digestion and high-pressure acid digestion.

Acid digestions of geological samples are not without challenges. Complete digestion of rock samples with a high proportion of refractory phases such as zircon, rutile or garnet can be difficult and typically require acid attack in closed-vessel PTFE bombs at high temperature for multiple days. Hydrofluoric acid (HF), an effective acid for breaking Si-O bonds, is commonly combined with other acids (e.g., $\mathrm{HNO}_{3}, \mathrm{HClO}_{4}, \mathrm{HCl}$ ) to break down geological samples, with the choice of acid mixtures strongly dependent on sample matrix. The formation of insoluble fluoride complexes can present a major challenge to achieving full dissolution. Insoluble phases (e.g., $\mathrm{AlF}_{3}, \mathrm{MgF}_{2}, \mathrm{CaAlF}_{5}$ ) typically incorporate large proportions of trace elements and lead to low sample recoveries and inaccurate solution analyses.

The importance of complete sample digestion in order to obtain accurate analytical results has been demonstrated by several recent investigations. Zhang et al. (2012) investigated the capabilities of $\mathrm{HF}$ and $\mathrm{HF}-\mathrm{HNO}_{3}$ in high-pressure digestion systems, or PTFE-lined stainless steel bombs. They focused on the complete digestion of felsic rocks, which have a reputation of being difficult to digest completely due to the presence of refractory phases such as zircon. Several parameters, such as $\mathrm{HF}-\mathrm{HNO}_{3}$ ratio, digestion time, digestion temperature, sample size, and presence/absence of insoluble fluorides were systematically evaluated through solution, multi-element ICP-MS analysis of the felsic USGS reference material GSP-2. For comparison, a series of reference materials covering the compositional spectrum of igneous rocks (basic-intermediate-acidic) plus two common sedimentary rock reference materials (shales) were also digested and evaluated using the same analytical strategy.

Variations on three basic digestion procedures (A: $1 \mathrm{ml} \mathrm{HF}+1 \mathrm{ml} \mathrm{HNO}$; B: $1 \mathrm{ml} \mathrm{HF}$; $\mathrm{C}: 2$ $\mathrm{ml} \mathrm{HF}$ ) were used for the decomposition of the rock reference materials. The final solution of each experimental digestion product were analysed for elemental concentrations of 37

This article is protected by copyright. All rights reserved. 
isotopes using an Agilent 7700X ICP-MS. Using $\mathrm{Zr}$ recovery as an approximation for refractory phase digestion, Zhang et al. (2012) conclude that using HF alone is the most efficient and cost effective method for attacking felsic rock samples. Results demonstrate that for complete digestion of a rock sample with $<100 \mathrm{mg}$ mass, it would take 8-12 hours at $190^{\circ} \mathrm{C}$ in a closed high-pressure vessel for complete $\mathrm{Zr}$ recovery. It was also found that sequential addition of $\mathrm{HNO}_{3}(0.2-6 \mathrm{ml})$ to the $\mathrm{HF}$ acid digestion solution lowered the digestion capability of the $\mathrm{HF}$, as noted by the decreased recovery of $\mathrm{Zr}$ with increasing amounts of $\mathrm{HNO}_{3}$ to the acid mixture.

The presence of insoluble fluoride phases were observed in the final solution for all experimental digestions using a $>100 \mathrm{mg}$ test portion, regardless of digestion solution used. Low recoveries of $\mathrm{Nb}$ and $\mathrm{Ta}$ suggest that a second $\mathrm{HClO}_{4}$ acid attack for samples $>100 \mathrm{mg}$ suppresses, but does not eliminate, fluoride formation. Zhang et al. (2012) conclude that HF alone can be used successfully in high-pressure digestion of a range of igneous rock compositions. They note that it is important that sample aliquots are $<100 \mathrm{mg}$ of ultra-fine fractions $(<30 \mu \mathrm{m})$, which raises the question of how representative a small sample can be, knowing that most reference materials have not been tested for homogeneity at the milligram scale (Cotta et al. 2007).

The formation of insoluble fluorides during $\mathrm{HF}-\mathrm{HNO}_{3}$ digestion is controlled by the proportions of $\mathrm{Al}: \mathrm{Mg}: \mathrm{Ca}$ in the sample, specifically the $[(\mathrm{Mg}+\mathrm{Ca}) / \mathrm{Al}]_{\mathrm{M}}$ (Takei et al. 2001). By adding $\mathrm{Mg}$ to the sample prior to digestion so $[(\mathrm{Mg}+\mathrm{Ca}) / \mathrm{Al}]_{\mathrm{M}} \approx 1$, Takei et al. (2001) were able to suppress the formation of $\mathrm{AlF}_{3}$, the most resistant fluoride formed during high temperature digestion of felsic silicate rocks. Recognising that the formation of insoluble fluoride phases is a major challenge to obtaining full sample recovery using traditional HF$\mathrm{HNO}_{3}$ digestion protocol, Cotta and Enzweiler (2012) incorporated the Mg-addition technique of Takei et al. (2001) into a new $\mathrm{HF}-\mathrm{HNO}_{3}$ digestion procedure using a High Pressure Acid System (HPA-S), which had been previously used successful for the digestion of biological samples (e.g., Amarasiriwardena et al. 1994) and platinum-group element bearing minerals in geological materials (e.g., Meisel et al. 2001).

During development of the HPA-S method, geological reference materials of both granitic and basaltic composition were used to evaluate the effect of digestion temperature, $\mathrm{HClO}_{4}$ addition and $\mathrm{Mg}$-addition prior to sample dissolution; the concentrations of 41 trace elements were determine in the final digestion solution. Element concentrations were measured using a Thermo Scientific X series ICP-QMS. The recovery results were then compared with recovery data from digestions of the same materials using traditional $\mathrm{HF}-\mathrm{HNO}_{3}$ digestion techniques (sintering, open vessel acid decomposition, closed vessel acid decomposition).

This article is protected by copyright. All rights reserved. 
The results of Cotta and Enzweiler (2012) show complete recovery ( $\pm 10 \%$ ) of most measured elements. Fluoride precipitation was inhibited using the HPA-S by finding the appropriate digestion temperature, adding $\mathrm{Mg}$ to samples prior to digestion and evaporation with $\mathrm{HClO}_{4}$. The HPA-S was demonstrated to be well suited for the determination of trace elements in geological samples with both mafic and felsic compositions as well as felsic samples with a high proportion of acid-resistant phases.

An alternative to traditional $\mathrm{HF}-\mathrm{HNO}_{3}$ sample digestion procedures is the open-vessel acid digestion method using ammonium fluoride $\left(\mathrm{NH}_{4} \mathrm{~F}\right)$ presented by $\mathrm{Hu}$ et al. (2013). $\mathrm{NH}_{4} \mathrm{~F}$ is a solid reagent with a boiling point of $260^{\circ} \mathrm{C}$ that can be purified using a PFA sub-boiling purification system prior to use for sample digestion. The high boiling point of $\mathrm{NH}_{4} \mathrm{~F}$ enables use of a high temperature, open-vessel (Savillex screw-top Teflon vials) acid digestion method, eliminating the use of high-pressure PTFE digestion bombs and HF. Because $\mathrm{NH}_{4} \mathrm{~F}$ has not been routinely used to dissolve geological materials, Hu et al. (2013) presented a systematic study of $\mathrm{NH}_{4} \mathrm{~F}$ for the decomposition of the felsic geological reference material GSP-2. Using an open-vessel digestion method they document the effects of digestion temperature, digestion time, addition of nitric acid, amount of $\mathrm{NH}_{4} \mathrm{~F}$ used, presence of insoluble fluorides and $\mathrm{NH}_{4} \mathrm{~F}$ purification.

Geological reference material USGS GSP-2 (granodiorite) was chosen to evaluate the decomposition capabilities of $\mathrm{NH}_{4} \mathrm{~F}$ because of its high $\mathrm{Zr}$ content $\left(580 \mu \mathrm{g} \mathrm{g}^{-1}\right.$ ). To further test the accuracy and precision of the $\mathrm{NH}_{4} \mathrm{~F}$ dissolution method, eleven additional geological reference materials covering a range of igneous rocks, shale and soils were also analysed. Multi-element analyses of experimental digestion products were conducted on acidic solutions, using an Agilent 7700x ICP-MS. Analytical precision of measured element concentrations was generally better than $10 \%$ (1 RSD) for all of the reference materials analysed. The accuracy of measured elemental concentrations was better than $10 \%$ for most elements.

The optimised procedure involves adding $\mathrm{NH}_{4} \mathrm{~F}$ and sample powder $(<74 \mu \mathrm{m})$ into a screw top PFA vial at a ratio of 6:1 $\left(\mathrm{NH}_{4} \mathrm{~F}\right.$ :sample). Complete digestion of refractory minerals using the $\mathrm{NH}_{4} \mathrm{~F}$ method is controlled by the combination of time and temperature, with temperature being a critical factor. The proportion of refractory mineral zircon digested controls the recovery of $\mathrm{Zr}$. A digestion time of $1.5 \mathrm{hr}$ and temperature of $250{ }^{\circ} \mathrm{C}$ was found to be optimal, which is approximately twelve times faster than traditional $\mathrm{HF}-\mathrm{HNO}_{3}$ digestion using a high-pressure PTFE digestion bomb at $190{ }^{\circ} \mathrm{C}$. Hu et al. (2013) discovered that addition of $\mathrm{HNO}_{3}$ has a negative effect on the digestion capacity of $\mathrm{NH}_{4} \mathrm{~F}$ : $\mathrm{Zr}$ recovery decreased with the addition of just $0.2 \mathrm{ml} \mathrm{HNO}_{3}$. Perhaps the greatest advantage of the $\mathrm{NH}_{4} \mathrm{~F}$

This article is protected by copyright. All rights reserved. 
digestion protocol is that no insoluble fluorides were found during the digestion of even 200 mg samples. Limiting fluoride precipitation is key to obtaining accurate trace element data for silicate rock samples. Results indicate this is possible using $\mathrm{NH}_{4} \mathrm{~F}$ without sacrificing time, money, or sample homogeneity.

\section{Performance of new generation MC-ICP-MS instruments}

The general trend in ICP-MS technology is toward more sensitive instruments to maximise measurement accuracy and precision while minimising the sample volume being analysed in case of solution analyses; in the case of in situ analyses improved spatial resolution is a key factor. The two manufacturers of multi-collector instruments have both produced secondgeneration instruments (Thermo Scientific - Neptune Plus; Nu Instruments - Nu Plasma II ES) or have made upgrade packages available for existing multi-collector instruments, with new technology aimed at improved sensitivity. Examples of new hardware to increase overall instrument sensitivity include improvements in ion transmission by redesign of the ICP interface and ion extraction region (e.g., cone geometry and source lens), improved vacuum within the interface region, the development of compact ion counting detectors installed within the Faraday detector array and the installation of low noise amplifiers with $10^{12} \Omega$ resistors for improved signal/noise ratios on Faraday detectors at low intensity signals.

Zhang et al. (2014) demonstrated the capabilities of the Thermo Scientific Neptune Plus MCICP-MS by measuring $\mathrm{Pb}$ isotopes in melt inclusions in olivine by laser ablation. Their ion counting method takes advantage of the new compact discrete dynode (CDD) detectors that can be installed within the focal plane of the mass analyser of the MC-ICP-MS. The optional CDD multipliers replace the Channeltron continuous dynode ion counters that were installed as an option on the original Neptune instrument. The CDD multipliers are expected to give improved stability and linearity over a wider dynamic range (up to $10^{6} \mathrm{cps}$ ).

The Neptune Plus MC-ICP-MS used for the in situ $\mathrm{Pb}$ isotope measurements has 8 ion counters installed within the detector block: Five CDD multipliers attached to Faraday cup L4 to collect isotopes ${ }^{202} \mathrm{Hg},{ }^{204} \mathrm{~Pb},{ }^{206} \mathrm{~Pb},{ }^{207} \mathrm{~Pb}$, and ${ }^{208} \mathrm{~Pb}, 2$ CDDs attached to Faraday cup $\mathrm{H} 3$ to collect ${ }^{232} \mathrm{Th}$ and ${ }^{235} \mathrm{U}$, and $1 \mathrm{CDD}$ attached to $\mathrm{H} 4$ to measure ${ }^{238} \mathrm{U}$. Using this multi-ion counting geometry and a Resonetics RESOlution M-50 $193 \mathrm{~nm}$ ArF excimer laser, Zhang et al. (2014) were able to achieve precisions on ${ }^{20 \mathrm{X}} \mathrm{Pb} /{ }^{204} \mathrm{~Pb}$ ratios better than $1.3 \%$ (2RSD) and precisions for ${ }^{20 \mathrm{X}} \mathrm{Pb} /{ }^{206} \mathrm{~Pb}$ ratios better than $0.23 \%$ (2RSD) with a ${ }^{208} \mathrm{~Pb}$ signal intensity > 200,000 cps. These results are similar to those achieved by Souders and Sylvester (2008) for in situ $\mathrm{Pb}$ isotope ratio measurements using Channeltron ion counters on the original Neptune instrument. The data reported by Zhang et al. (2014) is significant because it was collected at

This article is protected by copyright. All rights reserved. 
much higher spatial resolution (24-45 $\mu \mathrm{m}$ laser spot for Zhang et al. (2014) vs. 49-99 $\mu \mathrm{m}$ laser spot for Souders and Sylvester (2008)), representing a significant decrease in the volume of sample consumed while achieving similar levels of accuracy and precision. Zhang et al. (2014) did not address the linearity, short- or long-term stability of the CDD electron multipliers, which still remain to be demonstrated.

Two new features to enhance instrument sensitivity, now standard on the Neptune Plus, are a new 'Jet' sample cone and a large dry interface pump $\left(100 \mathrm{~m}^{3} / \mathrm{h}\right.$ pumping speed $)$ that improves pumping capacity at the interface. Several investigations, including Zhang et al. (2014), have reported improved sensitivity using the new Jet sample cone in combination with either the $\mathrm{H}$ or $\mathrm{X}$ skimmer cone when compared with the conventional sample cone and $\mathrm{H}$ skimmer cone set-up. For in situ $\mathrm{Pb}$ isotope measurements in silicate glass reference materials, the measured ${ }^{208} \mathrm{~Pb}$ signal intensity more than doubled after the Jet sample $+\mathrm{X}$ skimmer cone combination was installed. The repeatability of in situ $\mathrm{Pb}$ isotope ratios improved by at least a factor of two. Corresponding sensitivity increases were observed for $\mathrm{Hf}, \mathrm{Yb}$ and Lu during in situ MC-ICP-MS Hf isotope measurements (Hu et al. 2012) and for Nd during solution MC-ICP-MS analysis (Newman 2012).

Gases such as $\mathrm{N}_{2}$ or $\mathrm{H}_{2}$ have been shown to increase instrument sensitivity when added to one of the gas flows into the ICP (e.g., Iizuka and Hirata 2005, Guillong and Heinrich 2007). $\mathrm{N}_{2}$ addition has been documented to improve precision and accuracy of LA-MC-ICP-MS Hf isotope determination in zircon (Iizuka and Hirata 2005). The relative merits of three different cone combinations (1: standard sample $+\mathrm{H}$ skimmer; 2: standard sample $+\mathrm{X}$ skimmer; 3: Jet sample $+X$ skimmer) plus the addition of $\mathrm{N}_{2}$ to the central gas flow rate of the ICP on the accuracy and precision of in situ Hf isotope ratio analysis of zircon was explored by $\mathrm{Hu}$ et al. (2012) using a Neptune Plus and a GeoLas $193 \mathrm{~nm}$ ArF excimer laser ablation system (44 $\mu \mathrm{m}$ laser spot). Analytical precision is strongly dependent on signal intensity, so flow rates for both the admixed $\mathrm{N}_{2}$ and Ar were optimised for maximum ${ }^{180} \mathrm{Hf}$ sensitivity for each of the 3 cone combinations. Signal sensitivities for ${ }^{173} \mathrm{Yb},{ }^{175} \mathrm{Lu}$ and ${ }^{180} \mathrm{Hf}$ prior to the addition of $\mathrm{N}_{2}$ improved 1.4 times using the standard sample $+\mathrm{X}$ skimmer, and 2.5 times using the Jet sample $+\mathrm{X}$ skimmer, when compared with the standard sample $+\mathrm{H}$ skimmer cone set-up. In the presence of $\mathrm{N}_{2}$, the relative sensitivity was found to be element specific with $\mathrm{Hf}$ observed to have greater signal enhancement in the presence of $\mathrm{N}_{2}$ than either $\mathrm{Yb}$ or Lu. ${ }^{176} \mathrm{Hf} /{ }^{177} \mathrm{Hf}$ ratios in zircon reference materials 91500 and Mud Tank were measured using each cone configuration and corresponding optimum Ar makeup gas flow rate for $\mathrm{N}_{2}$ flow rates of $0 \mathrm{ml} \mathrm{min}{ }^{-1}, 2 \mathrm{ml} \mathrm{min}{ }^{-1}$ and $4 \mathrm{ml} \mathrm{min}{ }^{-1}$. Results for the ${ }^{176} \mathrm{Hf} /{ }^{177} \mathrm{Hf}$ ratios for the standard sample $+\mathrm{H}$ skimmer cone set up and the standard sample $+\mathrm{X}$ skimmer cone set up, both with and without $\mathrm{N}_{2}$ addition, were within acceptable limits of accuracy and

This article is protected by copyright. All rights reserved. 
precision. The ${ }^{176} \mathrm{Hf} /{ }^{177} \mathrm{Hf}$ ratios for the Jet sample $+\mathrm{X}$ skimmer in the presence of $\mathrm{N}_{2}$ were also acceptable. However, Hu et al. (2012) found large deviations (410 ppm for 91500; 470 ppm for Mud Tank) in Hf isotope ratios relative the reference values when using the Jet sample $+\mathrm{X}$ skimmer without nitrogen addition. Because the deviation was not a linear function of mass, it could not be corrected using standard mass fraction laws. Fortunately, the non-linear mass-dependent fractionation of $\mathrm{Hf}$ isotope ratios can be suppressed with the addition of $\mathrm{N}_{2}$ to the central channel (Ar make-up) gas. It was found that the non-linear massdependent fractionation exhibited by the Jet sample + X skimmer was related to the Ar makeup flow rate and not the result of improper corrections for isobaric interferences of ${ }^{176} \mathrm{Yb}$ and ${ }^{176} \mathrm{Lu}$ on ${ }^{176} \mathrm{Hf}$.

The origins of this non-linear mass dependent fractionation are not fully understood. Increased analytical sensitivity for $\mathrm{Nd}$ isotope measurements and non-linear mass dependant fractionation on $\mathrm{Nd}$ isotope ratios are not instrument specific, having been observed using enhanced sensitivity, modified geometry cones on the Neptune Plus, $\mathrm{Nu}$ Plasma HR and $\mathrm{Nu}$ Plasma 1700 MC-ICP-MS instruments (Newman et al. 2009, Newman 2012). Such nonlinearity was attributed to increased oxide formation when high sensitivity cones are used. A small amount of $\mathrm{N}_{2}$ added to the carrier gas during LA-MC-ICP-MS analysis can reduce oxide formation (Nesbitt et al. 1997, Hu et al. 2008) and it is possible that the $\mathrm{N}_{2}$ addition to the central channel gas reported by $\mathrm{Hu}$ et al. (2012) reduced both oxide formation and nonlinear mass dependent fractionation of the Hf isotopes; this could perhaps bring similar improvements for $\mathrm{Nd}$, but further work is needed.

Modifications for increasing ion transmission, and thereby enhancing sensitivity, have also been made for the latest generation Nu Plasma HR MC-ICP-MS. Changes have been made to both skimmer and sample cone geometries as well as a redesign of the interface chamber ('ES' interface) so as to improve the intermediate vacuum. There have also been modifications to the source lens, leading to improved ion transmission to the mass spectrometer. The vendor claims that with the new ES interface elemental sensitivity has improved at least 1.8 times over the entire mass range (Nu Instruments AN27). Cottle et al. (2013) reported measurements on the same Nu Plasma HR MC-ICP-MS instrument before (HR) and after (HR-ES) the enhanced sensitivity modifications were made. They found a 2.3 times increase in sensitivity for solution Hf isotope measurements and a 1.6-1.8 times improvement for laser analyses (Lu-Hf and $\mathrm{U}-\mathrm{Th}-\mathrm{Pb}$ in zircon) with no loss in accuracy. The improved signal/noise ratios resulted in $\sim$ two-fold improvement in analytical precision when the same laser ablation conditions were used for HR and HR-ES analyses.

This article is protected by copyright. All rights reserved. 


\section{Improved spatial resolution of laser analyses}

One of the most sought-after goals of LA-ICP-MS researchers is analysis with better spatial resolution (e.g., Johnston et al. 2009). Typical ablations are either spots that are 30 to $60 \mu \mathrm{m}$ wide and 20 to $50 \mu \mathrm{m}$ deep or line scans that are 10 to $30 \mu \mathrm{m}$ wide, and 2 to $10 \mu \mathrm{m}$ deep (Košler 2008). Ablations at this scale may mix components of a sample that have different origins, compositions or ages, particularly as the laser drills to depths beneath the sample surface that are not accessed by surface imaging methods (backscattered electron, cathodoluminescence, energy dispersive X-ray spectrometer) provided by scanning electron microscopy (SEM).

LA-ICP-MS system properties that are desirable for sampling on smaller scales include:

- high ICP-MS sensitivity for measuring small ablation volumes accurately and precisely;

- integrated imaging capabilities to avoid laser beam positioning on surface domains with heterogeneous compositions or ages;

- rapid washout ablation cells for separation of signals derived from successive laser pulses in a sample;

- proper data reduction protocols for "spectral skew" (non-simultaneous detection of isotope ratio pairs of interest including unknown element/known internal standard element ratios needed for concentration measurements) when using instruments recording analytes sequentially with a single detector or simultaneously using two or more detectors with different response times.

Significant improvements can still be made in each of these aspects of laser ablation systems.

Single shot ablations: It has been long recognised that perhaps the most desirable (albeit not the easiest) way to carry out spatially resolved laser analysis is by single shot ablation of a discrete, homogenous domain (e.g., Leach and Hieftje 2000, Liu et al. 2001, Cottle et al. 2009). A single ablation shot removes only the first micrometre or so of sample material so that SEM imaging can reliably document the homogeneity of the targeted ablation volume, even if a "pre-ablation" shot or two are used to remove sample surface contamination. Also, single shot ablation virtually eliminates laser-induced volatile/refractory element fractionation, which progresses as pit depth increases, degrading data quality for some applications, most notably U-Pb geochronology (Košler and Sylvester 2003). Nonetheless, the reliability of single shot analysis has remained suspect for many analysts because of potential effects from factors such as variable shot-to-shot laser irradiance, low total analyte counts (compared with conventional continuous, multi-pulse analyses), improper corrections for spectral skew, and different particle size distributions between samples and reference materials.

This article is protected by copyright. All rights reserved. 
The recent study of Cottle et al. (2012) gave a useful demonstration of the capabilities of single shot laser ablation using current state-of-the-art instrumentation, highlighting an application that may emerge as a niche area for the method: reconnaissance screening of U$\mathrm{Pb}$ ages of detrital zircon and monazite for provenance studies. Their experiments were carried out with a Nu Plasma HR MC-ICP-MS and Photon Machines 193nm ArF excimer laser system fitted with a two-volume Helex cell. Zircon and monazite RMs and unknown samples were measured for $\mathrm{U}-\mathrm{Pb}$ ages using both conventional continuous and single shot laser ablation. For single-shot analyses, the laser was fired (fluence of $3.1 \mathrm{~J} \mathrm{~cm}^{-2}$ ) once every $10 \mathrm{~s}$ and then moved to the next sample location. Ablation spots were $31 \mu \mathrm{m}$ wide in zircon and $8 \mu \mathrm{m}$ wide in monazite, with depths of $95-120 \mathrm{~nm}$.

Analyte signals were measured with a mixed Faraday $\left({ }^{238} \mathrm{U}\right) /$ ion counter $\left({ }^{206} \mathrm{~Pb}\right.$ and ${ }^{207} \mathrm{~Pb}$ ) detector array. Peak signal and decay (Tau) curves are offset in time between the two collectors types, because Faraday response is $\sim 0.2 \mathrm{~s}$ slower than that for the ion counters. Thus, total signals for each isotope must be integrated from before-shot baseline to after-shot baseline, thereby avoiding any systematic offset of the resulting isotopic ratios (Cottle et al. 2009). To avoid manual and subjective selection of peaks and backgrounds and so as to streamline data reduction, the authors wrote a new MatLab code (SLaPChron) that automatically identifies the baseline and the peaks, calculates total counts, performs corrections for mass bias and instrumental drift, normalises the unknowns to a primary reference material and calculates ages. (The code is not, unfortunately, published with the paper as an on-line electronic file supplement.)

When applied to detrital mineral populations, Cottle et al. (2012) found that the time required to determine $\mathrm{U}-\mathrm{Pb}$ zircon and $\mathrm{Th}-\mathrm{Pb}$ monazite ages (120 unknowns and some two dozen RMs) was reduced from $\sim 2 \mathrm{hr}$ using a protocol consisting of 15-40 s of continuous ablation for each spot to $\sim 20$ min using single shot ablations. There was only a modest increase in individual data point uncertainty with single shot ablations (from $2 \%$ to $~ 4 \%$ ) and no loss of sub-population identification (for detrital components representing $>5 \%$ of the total at the $95 \%$ confidence interval) compared with the continuous ablations.

The authors noted that the ability of the single shot method to screen large numbers of zircons rapidly would make it a powerful tool for identifying age populations of zircon of specific interest within large, diverse zircon populations such as magmatic grains in ash beds carrying large numbers of inherited grains, and Hadean grains in ancient quartzites dominated overwhelmingly by Archaean grains.

This article is protected by copyright. All rights reserved. 
Pulse-by-pulse data processing: Another strategy for improving spatial resolution of laser analyses involves processing data from a continuous ablation on a pulse-by-pulse basis. Two useful applications of this approach would be depth profiling as the laser progressively drills a pit into the target (Mason and Mank 2001) and 2- or 3-dimensional (2,3D) chemical/isotopic mapping as the laser makes linear tracks back and forth across a predefined area of the sample surface (Woodhead et al. 2007). The spatial resolution of these applications is, however, currently limited by the extent of mixing of pulse responses at the ablation site and in the ablation cell and transfer tubing. For instance, Rittner and Müller (2012) presented a mapping example using a scan speed of $67 \mu \mathrm{m} \mathrm{s}^{-1}$ with a 74- $\mu \mathrm{m}$ spot and a mass spectrometer sweep time of $0.496 \mathrm{~s}$, which resulted in a $33 \%$ overlap of ablation sites. Van Malderen et al. (2015) noted that washout times for commercial ablation cells are typically 1-2 s, whereas laser repetition rates above 1-2 Hz are commonly used for laser sampling, leading inevitably to overlap of pulse responses generated by sequential laser pulses. They reported the design of a low dispersion ablation cell and transfer tubing that achieves 99\% washout of ablated aerosols in only $\sim 6 \mathrm{~ms}$, providing a potential lateral resolution of $\sim 0.3 \mu \mathrm{m}$ during laser scanning. Wang et al. (2013) described a "tube cell" for laser ablation with wash-out times of $30 \mathrm{~ms}$. For depth profiling, Johnstone et al. (2013) examined methods for carefully determining laser-drilling rates, documenting the extent of non-linearity of drill rate with depth.

Tollstrup et al. (2012) described a different application for pulse-by-pulse data processing: measurement of $\mathrm{U} / \mathrm{Pb}$ isotopic ratios in discordant domains of single zircon grains for determining upper and lower intercept ages on $\mathrm{U}-\mathrm{Pb}$ Concordia diagrams. Here, the interest is not in mapping the distribution of the discordant domains in either 2- or 3dimensions within the grains, but instead simply defining their $\mathrm{U} / \mathrm{Pb}$ isotopic ratios to define a Discordia array as accurately as possible. The authors note that such discordant grains are commonly discarded in detrital zircon provenance and high-grade metamorphism studies, which might bias results.

Their analyses were carried out using a ThermoScientific Element XR ICP-MS and Photon Machines Analyte 193H laser system equipped with a dual volume HelEx ablation cell. A total of 350 scans of data were acquired during a $76 \mathrm{~s}$ acquisition period (25-30 s of gas background and $\sim 46-51 \mathrm{~s}$ of sample ablation), corresponding to $\sim 0.21 \mathrm{~s}$ for each data point, approximately equivalent to a laser pulse (for a $5 \mathrm{~Hz}$ repetition rate). Downhole fractionation factors for ${ }^{206} \mathrm{~Pb} /{ }^{238} \mathrm{U}$ and ${ }^{207} \mathrm{~Pb} /{ }^{235} \mathrm{U}$ were calculated for multiple zircon reference materials and the average ratios were used to correct down-hole $\mathrm{U} / \mathrm{Pb}$ fractionation of unknown zircon analyses back to the time of the start of ablation. The software program Isoplot (Ludwig 2009) was then used to calculate upper and lower intercept ages for a

This article is protected by copyright. All rights reserved. 
Discordia line fit to each data point obtained from a single laser ablation spot and, alternatively, for each consecutive set of five binned measurements. The method was applied to discordant zircons from metasedimentary xenoliths from a Miocene diatreme in the Sierra Nevada batholith. Upper and lower intercept ages calculated using all of the individual mass scan data (upper: $2780 \pm 40 \mathrm{Ma}$; lower: $106 \pm 38 \mathrm{Ma}$ ) were found to be identical within error to ages calculated using integrated data (the average of every five mass scans) (upper: $2810 \pm$ $64 \mathrm{Ma}$; lower: $125 \pm 43 \mathrm{Ma}$ ).

Although uncertainties on the reported ages are large for the discordant zircons, and their accuracy is difficult to assess without independent measurements by another technique such as isotope dilution-thermal ionisation mass spectrometry (ID-TIMS), the method could prove useful for some U-Pb zircon geochronology studies.

Tollstrup et al. (2012) split the ablated aerosol stream from their analyses so that a ThermoScientific Neptune Plus MC-ICP-MS measured Hf isotopes concurrently with the U$\mathrm{Pb}$ analyses. "Split-stream" analysis is becoming an increasingly popular way of linking analyses of $\mathrm{U}-\mathrm{Pb}$ age, $\mathrm{Hf}$ isotope and/or trace element composition of zircon domains directly (e.g., Kylander-Clark et al. 2013), although the trade-offs in reduced accuracy and precision compared with conventional single-stream analysis (e.g., Košler 2012) are not well established. As in many previous studies (e.g., Gerdes and Zeh 2009), the Hf isotopic compositions of the discordant domains in the zircon grains were uniform, indicating that variable $\mathrm{Pb}$-loss did not disturb the $\mathrm{Hf}$ isotopic ratios.

\section{Inter-laboratory comparison of in situ U-Pb zircon geochronology}

This year's review of LA-ICP-MS attests to the substantial interest of the geoscience community in applying the method to U-Pb zircon geochronology. It is therefore somewhat surprising that the quality of U-Pb zircon age data produced by LA-ICP-MS and its limitations have only rarely been examined in a systematic and rigorous fashion. An example is the study of Košler et al. (2013), which compared U-Pb age data from eight LA-ICP-MS and two SIMS laboratories on 1 inch round epoxy mounts of selected mixtures of grains from six zircon RMs of known ID-TIMS age, simulating the equivalent of a detrital zircon population.

The study addressed several important issues dealing with the accuracy and precision of U-Pb zircon geochronology by LA-ICP-MS and SIMS. First, in general terms, the accuracy of the in situ methods is $\sim 2 \%$, as determined by comparison with ID-TIMS ages for the same RMs. Certainly the $2 \%$ estimate is a function of the number of grains analysed as well as the $\mathrm{Pb}$

This article is protected by copyright. All rights reserved. 
concentrations in the grains (which is in turn a function of $U$ content and age of the RM). The results of Košler et al. (2013) suggest that, at least for the LA-ICP-MS analyses, accuracy is poorer than $2 \%$ for grains with comparatively low $\mathrm{Pb}$ concentrations and where comparatively few grains of a particular age are analysed. Future work should attempt to quantify accuracy of U-Pb zircon analyses by LA-ICP-MS as a function of both $\mathrm{Pb}$ concentration and number of analyses.

The laboratories performed well at identifying all of the correct age populations of zircon in the sample. Even for two age populations that made up only 5\% and $3 \%$ of the zircon, all participating laboratories identified both populations after 40 and 70 analyses, respectively. This should give the community some confidence that the 75-100 analyses that are commonly reported for a detrital zircon sample is likely to have identified at least one grain from all populations present at the $3 \%$ level and above. Some concerns with the accuracy of age peak identification were identified however. Two laboratories reported two false age peaks, albeit from discordant analyses that may well have been discarded from the interpretation of an actual detrital zircon sample. Also, two age populations in the sample mount with a difference in age of only $5 \%$ were not well separated by half of the laboratories. The implication is that apparent single age populations with a broader range of ages than other populations in actual detrital zircon samples should be checked carefully to ensure that they do not represent mixtures of two or more discrete age populations.

A test of the ability of the participating laboratories to determine the relative proportions of the zircon age populations in the sample mount produced mixed results. Probability Density Plots (PDPs), which are the most common way that the spectrum of detrital zircon ages for a sample are reported today (e.g., in Isoplot; Ludwig 2009), were qualitatively similar for the results from all ten laboratories. Peak intensities in the PDPs for the two dominant age populations in the sample, each representing $24 \%$ of the zircon present, were biased toward the population with a greater proportion of larger $(>100 \mu \mathrm{m})$ grains, indicating some degree of non-representative sampling by the analysts. The bias in the peak intensities for the two dominant age populations is also a result of the way that they are calculated in a PDP, being based both on the relative abundance and precision of age determinations. Vermeesch (2012) discussed this problem and suggested that Kernel Density Estimation (KDE), which does not explicitly take into account the analytical uncertainties, is a more appropriate way of plotting detrital zircon age results with respect to estimating relative abundances. His paper provided software to make KDE plots, and it would be instructive for future studies to compare detrital age populations using both the PDP and KDE approaches.

This article is protected by copyright. All rights reserved. 
Finally, Košler et al. (2013) noted that there was no obvious improvement in the accuracy and precision of LA-ICP-MS U-Pb zircon data collected using multi-collector or singlecollector magnetic sector ICP-MS versus quadrupole ICP-MS and an $193 \mathrm{~nm}$ excimer laser versus a $213 \mathrm{~nm} \mathrm{Nd-YAG} \mathrm{laser,} \mathrm{as} \mathrm{might} \mathrm{have} \mathrm{been} \mathrm{expected} \mathrm{based} \mathrm{on} \mathrm{the} \mathrm{theoretical}$ capabilities of the instrumentation. The authors attributed this result to the strong control that treatment of $\mathrm{U} / \mathrm{Pb}$ fractionation has on data quality, and suggested that improved (and more uniform) data handling and uncertainty propagation routines are needed (e.g., Horstwood 2008, Ulianov et al. 2012).

\section{New data processing software}

Data handling for U-Th-Pb analyses: LA-ICP-MS U-Pb age analyses for accessory minerals produce large data sets of time-resolved analyte count rates. In broad terms, there are two tasks involved in processing such data. In the first, intervals of analyte count rates during ablation are selected and then (1) corrected for the gas background and the isobaric interference of ${ }^{204} \mathrm{Hg}$ on ${ }^{204} \mathrm{~Pb}$, (2) corrected for laser-induced fractionation of $\mathrm{U} / \mathrm{Pb}$ and the presence of common $\mathrm{Pb}$ (if the measured ${ }^{204} \mathrm{~Pb}$ is significant) and (3) converted to ${ }^{206} \mathrm{~Pb} /{ }^{238} \mathrm{U}$, ${ }^{207} \mathrm{~Pb} /{ }^{235} \mathrm{U},{ }^{208} \mathrm{~Pb} /{ }^{232} \mathrm{Th}$ and ${ }^{207} \mathrm{~Pb} /{ }^{206} \mathrm{~Pb}$ ratios, which are calibrated against the known values of these ratios in one or more reference materials analysed along with the unknown minerals; appropriate analytical uncertainties must be propagated to the final corrected ratios. The second task involves calculating ${ }^{206} \mathrm{~Pb} /{ }^{238} \mathrm{U},{ }^{207} \mathrm{~Pb} /{ }^{235} \mathrm{U},{ }^{208} \mathrm{~Pb} /{ }^{232} \mathrm{Th}$ and ${ }^{207} \mathrm{~Pb} /{ }^{206} \mathrm{~Pb}$ ages and their uncertainties from the respective ratios that can then be displayed on Wetherill, TeraWasserburg and/or 3D U-Th- $\mathrm{Pb}$ Concordia diagrams, or as weighted averages, histograms and PDPs.

More than a dozen data reduction programs are in use for the first task, including some summarised in Sylvester (2008) and more recent workshops (Horstwood et al. 2010, Bowring et al. 2013). The lack of consensus as to which methodologies in these programs are most appropriate has almost certainly led to biases between laboratories for U-Th- $\mathrm{Pb}$ age data reduction (Košler et al. 2013). In stark contrast, the Excel-based Isoplot program of Ludwig (2009) is used almost exclusively for the second task. Here, the problem is that Isoplot is no longer being updated to retain full functionality with successive versions of Excel.

In this context, Petrus and Kamber (2012) developed a data reduction scheme (DRS) for Iolite (Paton et al. 2011), which is one of the more popular software packages used for the first task above. The new DRS, VizualAge calculates ${ }^{207} \mathrm{~Pb} /{ }^{206} \mathrm{~Pb}$ ages and common $\mathrm{Pb}$ corrections for each time-slice of raw data. More importantly it displays the data on the same type of plots made by Isoplot but in "live" fashion for the Concordia diagram so that the user

This article is protected by copyright. All rights reserved. 
can visualise the results as the selected interval of the analyte signal is adjusted. This allows discrete zones of discordance, inheritance and rim overgrowths within the ablated zone of the grain to be identified more easily. The new DRS is a significant advance because it combines the two data reduction tasks described above within a single software package.

More recently, Chew et al. (2014) presented a modified version of the VizualAge (VizualAge_UcomPbine) that can correct for variable amounts of common $\mathrm{Pb}$ in any $\mathrm{U}-\mathrm{Pb}$ accessory mineral $\mathrm{RM}$ as long as the material is concordant in the $\mathrm{U} / \mathrm{Pb}$ (and $\mathrm{Th} / \mathrm{Pb}$ ) systems following common $\mathrm{Pb}$ correction. Common $\mathrm{Pb}$ correction can be undertaken using the ${ }^{204} \mathrm{~Pb}$, ${ }^{207} \mathrm{~Pb}$ or ${ }^{208} \mathrm{~Pb}$ methods on raw data files from all commonly used multi-collector and singlecollector ICP-MS instruments. The DRS is open-source, freeware but the new version of Iolite (v3) is a commercial product.

Imaging Software: As noted earlier in this review, 2 and 3D chemical/isotopic mapping of minerals is an application of much interest in the geosciences (Woodhead et al. 2007). Chemical mapping by LA-ICP-MS was first developed for the biological and medical sciences (Becker et al. 2010). In that field, software is available to construct images from raw data obtained by scanning a sample line-by-line and further analyse those images by standard image analysis (Osterholt et al. 2011).

Two papers recently described imaging software for geoscience applications. Paul et al. (2012) described a module for Iolite referred to as CellSpace that creates images by synchronising the position on the sample with the measured mass spectrometer data, which is plotted as a circular spot representing the size of the area ablated. Individual 2D maps for each element or isotope ratio of interest can be created. Laser ablation data can be plotted over optical or scanning electron microscope images of the sample.

Rittner and Müller (2012) presented an open-source, freeware software package (LAICPMS) for the $\mathrm{R}$ language for statistical computing, which can also make $2 \mathrm{D}$ element maps of LA-ICP-MS data. A difference with CellSpace is that the LAICPMS software uses an empirical cumulative density function for optimised colour coding of the maps rather than a linear or logarithmic scale, maximising the visibility of element-specific detail.

\section{Advances in plasma source emission spectroscopy}

(Contribution by: K. Linge)

This article is protected by copyright. All rights reserved. 


\section{Plasma source emission spectroscopy in geochemistry}

Since the mid-1960s, inductively coupled plasma atomic emission spectroscopy (ICP-AES) has made major contributions to geochemistry by providing major, minor and trace element analysis of rocks and minerals. Since commercialisation in the mid-1970s, ICP-AES has been the instrument of choice for many laboratories, especially for its ability to determine between twenty to sixty elements within 2-3 min. Compared with flame AAS, ICP-AES provides lower detection limits, multi-element capability, a wider linear dynamic range and it is able to analyse samples with higher total dissolved solids than ICP-MS with better precision. As an established analytical technique, advances in ICP-AES instrumentation are now rare. While other plasma sources and types have been developed and tested (Jankowski and Reszke 2013, Montaser et al. 1992), commercial hardware is focused solely on instruments using argon based ICP. Recently, however, a commercial microwave plasma (MP) AES system has been introduced onto the market, which appears to provide an attractive alternative to flame AAS and ICP-AES. The MP-AES system utilises a relatively new design of plasma torch, utilising nitrogen gas, previously reported by Hammer (2008), and several geochemical applications of the instrument have now been reported.

\section{Recent applications for commercial MP-AES}

Developments in instrumentation for MP sources have been reviewed by Jankowski and Reszke (2013). Historically, plasmas have used energy from microwave electric field, leading to very thin, rod-like plasmas. Such plasmas are not well suited for the introduction of a wet aerosol, which undergoes rapid heating and expansion as it approaches the plasma, preventing entrainment of the sample stream into the plasma. The development of annular plasmas has been particularly important for optimising plasma-aerosol interactions, where the central channel must be large enough to allow sample injection, but small enough to ensure good thermal coupling. While MP-AES systems capable of accepting a wet aerosol have existed since the early 1990's, they are more susceptible to matrix interferences than argon ICP systems, in general leading to inferior detection limits. In contrast to previously reported systems, the newly commercialised MP-AES system couples energy from the microwave magnetic field rather than the microwave electric field, using a hollow rectangular metal section called a waveguide (Hammer 2008). The magnetic field is reflected at the end of the waveguide at a closed 'short-circuit' end, which is designed to create standing waves within the guide, and a magnetic field maximum at half a wavelength from the short-circuited end. The quartz torch is positioned within the magnetic field maximum and obstructions placed within the waveguide alter the field patterns, acting as capacitors or inductors. The harmonically oscillating magnetic field $(2450 \mathrm{MHz})$ generated by the conductor induces an electric field in which the plasma electron are accelerated (Jankowski and Reszke 2013). The

This article is protected by copyright. All rights reserved. 
resulting plasma exists as a hollow tube that can be held away from the torch wall by a layer of fast flowing sheath gas (Figure 1). Using argon as the plasma gas resulted in a plasma thickness of only a few $\mathrm{mm}$, and poor coupling to the central channel. However, plasma thickness increased when using nitrogen as the plasma gas, due to its reduced electron density and plasma conductivity. Increased power was required to sustain the nitrogen plasma $(1 \mathrm{~kW})$ compared with the argon plasma $(200 \mathrm{~W})$. The coupling between the sample and plasma was also improved by distorting the shape of the plasma by combining an axial magnetic field with a transverse electric field, which creates a plasma with an elliptical cross section. This was achieved using a resonant iris near the torch.

$<<$ Figure 1 approximately here $>>$

The gas flows and torch used in the new MP-AES system are very similar to those used in ICP-AES, although the injector tip in the torch needs to be positioned closer to the plasma (Hammer 2008). Whereas ICP typically requires cooling through the copper induction coil, no additional cooling is required for the MP waveform cavity other than natural air convection, resulting in a maximum temperature $\sim 40{ }^{\circ} \mathrm{C}$ hotter than ambient temperature as the MP torch is efficiently cooled by the sheath gas flow. Theoretically the MP may be viewed either axially or radially through an opening in the cavity end wall that is designed to attenuate microwave energy. However, in practise, the commercial MP-AES system utilises axial viewing as this offers better sensitivity, with the viewing position able to be modified using an optical mirror (Donati et al. 2013).

The most sensitive emission lines for MP-AES are typically atom lines rather than ion lines (Hammer 2008), probably related to the lower temperature of the MP ( $5000 \mathrm{~K}$, Donati et al. 2013). While the background for ICP-AES includes both continuum and structured background signals, mostly in the visible region near $400 \mathrm{~nm}$, the MP-AES background is dominated by highly structured emission lines of $\mathrm{NO}$ and $\mathrm{N}_{2}$ in the UV region, which can influence limits of detection (LOD) for some wavelengths. Detection limits of the prototype MP-AES system for fourteen elements ranged between 0.09 and $140 \mathrm{ng} \mathrm{ml}^{-1}$. In comparison, an ICP-AES system using the same sample introduction system and polychromator achieved comparable LODs for $\mathrm{Ba}, \mathrm{Cr}, \mathrm{Cu}, \mathrm{Mo}, \mathrm{Ni}, \mathrm{Pb}$, and $\mathrm{Sr}$ (Hammer 2008). The ICP-AES system had better detection limits (by a factor of 5 or more) for As, Cd, Co, Mn, Se and Zn, while MP-AES had a better detection limit for Al by a factor of 5.7.

The new MP-AES instrument has been demonstrated using several different matrices, including rocks, ores and other geochemical materials (Balaram et al. 2014; Balaram et al. 2013), as well as ethanol and gasoline (Donati et al. 2013). Analyses of precious metals in

This article is protected by copyright. All rights reserved. 
several rock and ore reference materials utilised an aqua regia digestion without further separation or pre-concentration for Ag and Au analysis, whereas a NiS fire-assay followed by tellurium co-precipitation was used for Pd and Pt (Balaram et al. 2013). To minimise matrix effects and interferences, calibration was undertaken using solutions of identically prepared international precious metal reference materials, which is a common method of overcoming matrix effects in ICP-AES. Quantitative results were similar for Ag and Au when calibrated with either matrix-matched reference materials or when using aqueous calibrants; this was not the case for $\mathrm{Pd}$ and $\mathrm{Pt}$, although no hypothesis was given by the authors for these differences. All precious metal results compared closely to both the reference values and the analytical results from established techniques (e.g., flame AAS and ICP-MS) when calibrations were based on matrix matched calibrants. Balaram et al. (2014) also reported analyses of major, minor, and some trace elements in eight international rock, sediment and soil reference materials using MP-AES after either digestion utilising a multi-acid mixture in a closed vessel or lithium metaborate fusion in a glassy carbon crucible. In that study wavelengths were selected based on sensitivity, and were predominantly atomic lines. A study of interference effects from concomitant elements present in the samples found significant signal enhancements in the presence of alkali and alkaline earth elements. Such signal enhancements were attributed to a shift in the equilibrium between atom and ions, caused by reduced plasma temperature and increased electron density. Other major elements, such as $\mathrm{Ti}, \mathrm{Fe}, \mathrm{Al}$ and $\mathrm{Mn}$, had similar, but smaller enhancement effects on signals from atomic lines. LODs ranged from 0.1 to $33 \mu \mathrm{g} \mathrm{l} 1$, which is superior to flame AAS and approaches the LOD values typical of ICP-AES. Repeatabilities of $\leq 3 \%$ RSD were obtained for major and minor elements (Al, $\mathrm{Ca}, \mathrm{Fe}, \mathrm{K}, \mathrm{Mg}, \mathrm{Mn}, \mathrm{Na}, \mathrm{P}, \mathrm{Ti})$ and $\leq 6 \% \mathrm{RSD}$ for trace elements (Ba, Be, Cr, Cu, Ga, Hf, Li, Mo, Nb, Ni, Pb, Rb, Sc, Sr, Ta, Zr, Zn). There was very good agreement between concentrations determined by MP-AES and certified values for the reference materials.

Analyses of $\mathrm{Cr}, \mathrm{Ni}, \mathrm{Pb}$ and $\mathrm{V}$ in ethanol and gasoline by MP-AES utilised an external gas control module that introduce air into the plasma (Donati et al. 2013). This promoted the formation of $\mathrm{CO}$ and $\mathrm{CO}_{2}$, thereby suppressing $\mathrm{C}$ interferences and soot formation in the torch. Ethanol samples were diluted 10 -fold in $1 \% \mathrm{HNO}_{3}$ solution, while gasoline samples were prepared as micro-emulsions with aqueous $\mathrm{HNO}_{3}(20 \%)$, Triton X-100 and $n$-propanol. LODs for $\mathrm{Cr}, \mathrm{Ni}, \mathrm{Pb}$ and $\mathrm{V}(0.3-60 \mu \mathrm{g} / \mathrm{L})$ were lower than previously reported for ICP-AES, which may indicate that the lower sensitivity of MP-AES can be overcome in part by an optimisation of the sample introduction system or integration time. The linear dynamic range of such MP-AES analyses was between 1.3 and 3.5 orders of magnitude, close to that expected from flame AAS, but much poorer than ICP-AES (typically considered to have a

This article is protected by copyright. All rights reserved. 
linear dynamic range of 6 orders of magnitude). Accuracies for spiked ethanol and gasoline samples were generally within $10 \%$, even without matrix matched calibration materials.

\section{Future outlook for plasma source emission spectroscopy}

While MP-AES holds promise as an alternative analytical method to AAS and ICP-AES, it is still very much in its infancy, with only 9 publications in the Web of Science ${ }^{\mathrm{TM}}$ database reporting the use of the new MP-AES system up until the time of writing (September 2014). The new system is primarily being marketed as an AAS replacement; it is not yet clear how successfully it will be able to compete as an alternative for ICP-AES in geochemical applications. Hopefully, as the instrument is adopted by research organisations in particular, future publications will provide more detailed comparisons of MP-AES with AAS, ICP-AES and even ICP-MS. Despite this uncertainty, the MP-AES instrument has a number of obvious advantages for commercial use. Use of a nitrogen MP represents a considerable cost savings compared with an argon ICP, particularly as a nitrogen generator with air compressor can be used to supply the nitrogen plasma, effectively eliminating on-going gas costs. Compared to AAS, the nitrogen MP does not require the use of flammable gases such as acetylene, providing a safer laboratory environment. MP-AES is likely to find particular application in remote sites and mobile laboratories, where regular supply of analytical grade gases can be both difficult and expensive. Given the similarity of sample introduction systems between the MP-AES and conventional ICP-AES, it is anticipated sample introduction and sample preconcentration strategies already validated for ICP-AES will be equally robust for MP-AES, which would make MP-AES an even more attractive alternative technique.

\section{Advances in X-ray fluorescence methods}

(Contribution by L.P. Bédard and D. Savard)

\section{Overview of X-ray based methods}

$\mathrm{X}$-ray fluorescence (XRF) is a mature technique that has seen its most significant developments a few decades ago. Nonetheless, it remains an important tool for quantifying major elements and some trace elements in geomaterials. Now to be considered a fully matured method, XRF-based methods can be found in a variety of instruments such as microXRF (Figure 2A), portable XRF (PXRF), handheld XRF (HHXRF, Figure 2B), core scanners and as an attachment for electron beam instruments such as electron probe micro-analysis (EPMA) and scanning electron microscope (SEM), where characteristic X-rays are stimulated by electron bombardment (Table 1). Atoms excited by an X-ray beam will expel

This article is protected by copyright. All rights reserved. 
electrons. Outer shell electrons of the unstable atom will "fall" towards lower orbitals, resulting in the emission of X-ray photons that are characteristic of the element with a signal intensity that is a function of the abundance of the element in the excited volume. Two types of detectors are currently used: wavelength dispersive spectrometers (WDS) and energy dispersive spectrometers (EDS), where the EDS detection system in particular has seen major technical advances in the last decade with the introduction of the silicon drift detector (SDD). Sample preparation for laboratory XRF instruments involves the preparation of either glass disks or pressed pellets while microbeam instruments (EPMA, SEM, micro-XRF) generally employ polished samples or thin sections. HHXRF can be used to analyse prepared glass disks, pressed pellets or even unprepared samples in the field. In micro-XRF analysis the Xray beam is focussed through a polycapillary lens that produces a small beam $(\sim 50 \mu \mathrm{m})$, thereby providing a mapping tool for working at the $\mathrm{mm}$ to sub-mm scale. A recent review of the analytical field of XRF is presented in West et al. (2014). Synchrotron XRF is not included in this review.

\section{Recent publications based on X-ray methods}

A study by Pleßow (2013) provides an explanation for the difficulties in measuring $\mathrm{F}, \mathrm{Cl}$ and B by XRF. Repeated determinations of these analytes in rocks and soils yielded poor reproducibility: results were systematically drifting, often increasing but also decreasing in some cases. X-ray ionising radiation is known to induce diffusion of fluorine, boron and chloride in bombarded samples; Pleßow (2013) puts forward that the diffusion of radiolysis products toward the sample surface was the cause for poor reproducibly for these elements.

Methods capable of mapping phase assemblages in polished or thin sections using SEM combined with EDS XRF (Mineral Liberation Analysis (MLA from MINTEK) and Quantitative Evaluation of Minerals by SCANning electron microscopy (QEMSCAN®, a trade mark of FEI company) are popular techniques that are used quite intensively in mining geochemistry. Lund et al. (2013) described an analytical protocol that can be applied to iron ores for producing a modal mineralogy from XRF results. They based the element to mineral conversion on a set of linear expressions and least-square equations using EPMA mineral determinations, going on to validate the modal results against QEMSCAN® analyses. The authors claim that their modal estimates using XRF results gave equivalent results to those obtained using QEMSCAN® in 95\% of cases.

Portable and hand held XRF: Interest in field portable XRF has exploded in the fields of environment and mining exploration geoanalysis; in particularly HHXRF has contributed data towards hundred of papers over the past two years. The main themes of these publications have been instrument performance, calibration schemes and case studies. Here

This article is protected by copyright. All rights reserved. 
we note that the journal "Geochemistry: Exploration, Environment, Analysis" recently published a special issue devoted to HHXRF (August 2014, vol. 14). In terms of technical innovation, PXRF instrumentation is approaching a high level of maturity. The capacity to measure directly in the field opens new opportunities for alternative sampling strategies. The most interesting development is a paradigm change where an instrument producing lower quality results (PXRF or HHXRF) than large laboratory XRF instrumentation can be successfully used thanks to an improved sampling strategy (Ramsey and Boon 2012). If one considers sampling uncertainty as part of the total uncertainty budget, then the lower sampling uncertainty might compensate, in some cases, for higher instrumental uncertainty. In some cases, the HHXRF (in situ measurement) can be more "fit for purpose" than laboratory analyses (ex situ measurements). Ross et al. (2013, 2014a, 2014b) presented a series of publications on an integrated core logging system that determines volumetric magnetic susceptibility, density, chemical composition (using HHXRF) and mineral identification (using visible/near infrared spectrometry) on drill core specimens. They found that the analytical uncertainty provided by HHXRF is adequate, though corrections may be necessary as the measurement are done directly on core surface without sample pulverisation or any further preparation. Their more recent publications address HHXRF, where they evaluate the performance of one HHXRF instrument and explain their corrections (Ross et al. 2014a) while in the companion publication (Ross et al. 2014b) they provide a case study from the Matagami mining camp (Canada). An important issue they raise is that of mineralogical heterogeneity, which especially impacts samples that are coarser grain as compare to the HHXRF beam size. Not surprisingly, these authors confirm that when grain size approaches or exceeds the sampling size of the technique the overall "scatter" in the data increases, leading these authors to apply a three-point moving average to reduce the effects mineralogical variations. Ross et al. (2013, 2014a, 2014b) compared in situ results (HHXRF) with ex situ data and conclude that HHXRF can provide quite useful information.

Among the numerous case studies published using HHXRF applied to geomaterials, Le Vaillant et al. (2014) presented a well-documented case devoted to the exploration of nickel sulphide deposits. They developed a strict protocol to analyse chalcophile and lithophile elements, concluding that if a strict protocol is established and followed then HHXRF can deliver fit for purpose results. A look at other recent literature suggests that the determination of detection limits for HHXRF is not well understood by many users; Le Vaillant et al. (2014) are among the few authors who have proposed a realistic procedure for estimating overall analytical uncertainty.

Micro-XRF: Many acronyms for micro X-ray fluorescence instrumentation can be found in the scientific literature: micro-XRF, M-XRF, MXRF, $\mu$-XRF, $\mu$ XRF, etc., and these

This article is protected by copyright. All rights reserved. 
become even more numerous when the detector and/or source of X-rays is specified as in micro-EDXRF, $\mu$ EDX, micro-WDXRF, micro-SXRF, SR $\mu X R F$, etc. Finally, as three dimensions analyses can be achieved, the acronyms become even more varied: 3D-microXRF, 2D-micro-XRF, etc. Considering that the abbreviation "micro-XRF" appears to be the most commonly used in the literature, it will be used as a generic acronym in this review. Physical configurations of micro-XRF have multiplied significantly, mainly due to combining it with other techniques such as XANES (X-ray Absorption Near Edge Structure). One of the more interesting recent developments, confocal micro-XRF was used for 3D layer imaging by incorporating a second X-ray optical system in front of the energy dispersive Xray (EDX) detector. Having the foci of the incoming and outgoing optical systems coincide results in a small, well defined volume for the analysis. Such developments have meant that micro-XRF has been a very active field, with hundreds of publications devoted to the topic in the past two years; the year 2013 brought $\sim 600$ publications on the topic. Numerous reviews covering micro-XRF applications and experimental setups have been published recently, including West et al. (2014), Cnudde and Boone (2013), Holt et al. (2013), Smolek et al. (2014), Kanngießer et al. (2012), Huber et al. (2014), Smolek et al. (2012), Nakazawa and Tsuji (2013), Miot et al. (2014), Obst and Schmid (2014) and Dehlinger et al. (2013). Some of these publications are not devoted to geoscience topics, but they contain useful information and descriptions of interesting developments that might prove useful to geoscientists in the future.

The scope of this review of micro-XRF has necessarily been limited to those key developments that are most relevant to its application in the field of analytical geochemistry. Readers seeking more thorough or more general reviews of the micro-XRF technique should refer to the aforementioned publications. Jean-Soro et al. (2013) used micro-XRF to map elements in soils in an experiment where they doped soils to simulated car catalyst pollution in an urban environment. Their goal was to locate platinum-group elements in order to understand better their behaviour in the environment. Using micro-XRF maps, kaolinite was found to be the substance that adsorbs the most Pt followed by hematite and humic acid. Ichikawa and Nakamura (2014) used WDXRF with a $<1 \mathrm{~mm}$ diameter microbeam to determine major and trace element contents in siliceous samples as part of an archaeological investigation. They used lithium borate to prepare fused microdisks from $1.1 \mathrm{mg}$ of sample material. The main advantage reported by these authors was that WDXRF yielded reproducible data on such small samples, which is often a limiting factor in quantitative archaeology. Ichikawa and Nakamura (2014) were able to verify that their disk thickness was sufficient to satisfy the infinite thickness concept. Results on their small disks were compared with commonly used larger disk sizes and they reported reproducibilities better than 95\% and, using the established large disk method as a benchmark, accuracies better than $1 \%$ for

This article is protected by copyright. All rights reserved. 
$\mathrm{SiO}_{2}, \mathrm{Al}_{2} \mathrm{O}_{3}, \mathrm{TiO}_{2}, \mathrm{MnO}, \mathrm{K}_{2} \mathrm{O}$, better than $3.5 \%$ for $\mathrm{P}_{2} \mathrm{O} 5$ and $\mathrm{CaO}$, and better than $5 \%$ for $\mathrm{Fe}_{2} \mathrm{O}_{3}$ and $\mathrm{Na}_{2} \mathrm{O}$. Their sample technique could probably be easily modified for use with the more common polycapillary focussed micro-XRF. MacIntosh et al. (2014) presented a technique for analysing $\mathrm{Pu}$ in microfluidic samples, which they went on to test on two instruments: a high resolution monochromatic wavelength dispersive X-ray fluorescence and a commercial micro-XRF (Eagle III from EDAX Inc., Figure 2A). Because Pu is radioactive, Sr was used as a proxy for evaluating instrument sensitivity, yielding a detection limit of $\sim 5$ $\mu \mathrm{g} \mathrm{ml}^{-1}$ in the liquid sample. The accurate determination of solute contents in natural liquids is often of interest in geological and environmental studies. Devès et al. (2012) investigated the geochemistry of speleothems by combining micro-XRF and autoradiography imaging. They recorded the distribution of $\mathrm{U}, \mathrm{Ca}, \mathrm{Sr}$ and $\mathrm{Si}$ in speleothems, observing an inverse correlation between $\mathrm{Ca}$ and $\mathrm{U}$ and between $\mathrm{Ca}$ and $\mathrm{Si}$, with a significant correlation detected between $\mathrm{U}$ and $\mathrm{Si}$; these authors interpreted this correlation to be the result of a volcanic event that released uranium-rich ejecta that was easily soluble, thereby provided both $\mathrm{Si}$ and $\mathrm{U}$ sources for the speleothems. Considering the importance of speleothems as climate archives, micro-XRF studies may develop into a powerful tool both for understanding geochemical processes and for improved correlation of radiometric ages.

Characterising material heterogeneity is a major aspect in reference material development. However, many geochemical RM development projects do not attempt a detailed quantification of material heterogeneity at fine-scale sampling sizes appropriate for microanalytical work. Micro-XRF was used by Bédard and Néron (2014) to quantify heterogeneities in powdered reference materials. They used chemical maps of reference materials pressed into pellets to characterise the spatial distribution of heterogeneities in the RM, from which they could define a minimum test portion mass for representative results. Their approach could be applied to microbeam RMs such as glasses, where spatial heterogeneity is an important issue.

\section{Advances in TIMS ion detection}

(Contribution by: M. Horan)

In thermal ionisation mass spectrometry (TIMS) the element of interest is ionised from a heated filament, usually a refractory metal. Prior to mass spectrometric analysis, the element is purified from its matrix using wet chemical techniques. Modern TIMS instrumentation can analyse either positively or negatively charged ions and molecules; the ionised species is accelerated by a high voltage, focused into a collimated beam by ion optic components in the ion source region and deflected through a perpendicular magnetic field where the ion beam is separated by mass into its constituent isotopes. For higher ion beam intensities the individual

This article is protected by copyright. All rights reserved. 
isotopes are quantified by Faraday cups detectors; for lower intensities secondary electron multipliers (SEM) or Daly photomultiplier are generally used. Modern commercial TIMS, including the Thermo Fisher Triton, IsotopX Phoenix and $\mathrm{Nu}$ Instruments $\mathrm{Nu}$ TIMS, are equipped with an array of Faraday cups so that multiple isotopes can be simultaneously collected. Each Faraday cup detector employs a current amplifier equipped with a high-ohm feedback resistor, traditionally a $10^{11} \Omega$ resistor. The high precision capability of a Faraday cup detector using a $10^{11} \Omega$ resistor is, however, compromised by thermal noise at signal intensities below circa $10^{-12}$ amps, yet such ion currents are still too large for detection using an SEM. This section describes recent progress using higher resistance current amplifiers, expanding the ability of TIMS to provide precise quantification of lower intensity ion beams which may be imposed by very low isotopic abundances or small sample sizes.

Noise in the Faraday cup amplifiers (Figure 3) is the dominant factor limiting instrumental measurement uncertainty at low signal intensities in TIMS. The high-ohm feedback resistor in the amplifier is the main source of that noise, called Johnson Nyquist noise. The intensity of the noise scales with the square root of both the ambient temperature and the value of the resistor. For a given signal intensity, increasing the resistance by a factor of 10 , therefore, should improve the signal to noise ratio by a factor of more than 3 . Reproducibility of baselines, with integration times of several minutes, collected using $10^{12} \Omega$ resistors is typically 3-5 times better than using $10^{11} \Omega$ resistors (Koornneef, et al. 2013). The main disadvantage of using $10^{12} \Omega$ resistors is their significantly slower response time, requiring 3-5 times longer for decay of a signal, relative to $10^{11} \Omega$ resistors (Koornneef et al. 2013), along with greater temperature dependence of their resistance. For TIMS analyses performed in static mode (i.e., without peak hopping) on multiple Faraday cups, the slower response time can be regarded as only a minor issue.

$<<$ Figure 3 approximately here $>>$

Regarding the comparison of $10^{12}$ versus $10^{11} \Omega$ resistors, Koornneef et al. (2013) investigated measurement repeatability as a function of beam intensity for analyses of $\mathrm{Sr}$ and $\mathrm{Nd}$ reference samples. They found that if the intensity of the less abundant isotope was smaller than $100 \mathrm{mV}$, as measured on a $10^{11} \Omega$ resistor, then the internal precision of measured isotope ratios was improved by using $10^{12} \Omega$ resistors. Internal precision of a $4 \mathrm{mV}$ signal, for example, was improved by a factor of more than 2 by using a $10^{12} \Omega$ resistor. The repeatability for multiple analyses at a beam intensity of $3 \mathrm{mV}$ using $10^{11} \Omega$ resistors was improved by a factor of 3 , to $\pm 0.4 \%$ o $(2 s)$ when the amplifiers were swapped with $10^{12} \Omega$ resistors. This work suggests that employing a full array of detectors having $10^{12} \Omega$ resistors

This article is protected by copyright. All rights reserved. 
will allow resolution of $\mathrm{Nd}$ and $\mathrm{Sr}$ isotope ratios to the fourth decimal place in quantities of these elements as little as $100 \mathrm{pg}$.

Liu et al. (2014) compared results for static analyses of small quantities of Os using $10^{12} \Omega$ resistors with those obtained by conventional single collector analyses using a SEM in pulse counting mode. For many terrestrial studies, repeatability and internal precision of the ${ }^{187} \mathrm{Os} /{ }^{188} \mathrm{Os}$ ratio are acceptable at the $\pm 1-2 \%$ o level. In the study by Lui et al. (2014), baselines were acquired for $22 \mathrm{~min}$ on the Faraday cup detectors between one and a few times per day. Analyses of reference materials and samples were then conducted using a tenminute, static multi-collector method for Os data acquisition. This analytical protocol yielded a repeatability better than $\pm 0.6 \%$. Intensities equivalent to 1 to $2 \mathrm{mV}$ on $10^{11} \Omega$ resistors were achieved for sample sizes as low as $0.1 \mathrm{ng}$ Os. For Faraday cups equipped with $10^{12} \Omega$ resistors, a minimum sample size of $0.025 \mathrm{ng}$ of total Os appeared necessary to achieve uncertainties that were comparable to or better than SEM measurements.

Naumenko et al. (2013) re-evaluated the ${ }^{40} \mathrm{~K} /{ }^{39} \mathrm{~K}$ ratio used for $\mathrm{K}-\mathrm{Ar}$ and $\mathrm{K}-\mathrm{Ca}$ dating by employing two amplifier configurations having different feedback resistors. The current IUPAC recommendation for the isotopic abundance of ${ }^{40} \mathrm{~K}$ has an uncertainty of $\pm 0.35 \%$, which is the largest contributor to the overall uncertainty in K-Ar dates. The isotope composition of $\mathrm{K}$ is difficult to measure because mass dependent fractionation during analysis is large and because ${ }^{40} \mathrm{~K}$ is a very minor isotope of $\mathrm{K}(0.01 \%)$, requiring precise quantification of ion beams over a large dynamic range. The most accurate results obtained in this study used amplifiers with a $10^{10} \Omega$ resistor for ${ }^{39} \mathrm{~K}, 10^{12} \Omega$ for ${ }^{40} \mathrm{~K}$, and $10^{11} \Omega$ for ${ }^{41} \mathrm{~K}$, which optimised the performance of the amplifier assigned to each ion beam species. Separate measurement protocols controlled for mass dependent fractionation of the reference material during analysis, using either total evaporation or an in-run mass bias correction. These authors proposed a new ${ }^{40} \mathrm{~K} /{ }^{39} \mathrm{~K}$ ratio for SRM 985 that agrees with the previously accepted IUPAC value, but reduced its uncertainty to $\pm 0.05 \%$.

These early studies suggest that the use of Faraday cup current amplifiers with $10^{12} \Omega$ feedback resistors may help bridge the gap between traditional Faraday cup and SEM detection in TIMS. Such high resistance amplifiers offer two key advantages. First, for some low abundance TIMS work, an array of $10^{12} \Omega$ detectors may lessen the need for SEM collectors, which can require more tuning, longer analysis times and more frequent replacement. Second, a combination of Faraday cup detectors having different value feedback resistors may be useful for isotope characterisation of elements that have large ranges in their isotope abundances, such as some lower-mass elements or transition metals, and for some especially low-abundance isotopes, such as those produced by the p-process in

This article is protected by copyright. All rights reserved. 
nucleosynthesis. In such cases, the resistors could be tailored to a particular application. It remains to be seen, however, whether the additional dynamic range offered by high resistance current amplifiers also allows for sufficient flexibility in laboratories that must cater to a range of analytical needs.

\section{Advances in accelerator-based methods}

(Contribution by: R. Bugoi and S. Merchel)

\section{Overview of accelerator-based methods}

A brief introduction in accelerator-based methods has been given in the last GGR Biennial Review (Wiedenbeck et al. 2012). The review papers and books mentioned therein have been supplemented by reviews by Granger et al. (2013a) and Kutschera (2013a, b) for accelerator mass spectrometry (AMS) and by Zucchiatti and Redondo-Cubero (2014) for ion beam analysis (IBA).

AMS is mainly used for the detection of long-lived radionuclides; the most prominent example being radiocarbon $\left({ }^{14} \mathrm{C}\right)$. Radionuclides need to be enriched and chemically isolated from the sample matrix before they can be converted into a chemically stable form and placed in a Cs-sputter ion source, from which negative ions (molecules or elements) are extracted. The ions are injected into a tandem accelerator, where they gain MeV-energies and, by passing through "stripper" matter (gas or thin foil), transmute into multiple-positively charged ions, which are further accelerated towards the exit. All molecules are destroyed by this stripping process, thereby allowing isotope ratios to be measured from a much-simplified spectrum. Stable nuclides are detected using Faraday cups whereas the much, much less abundant radionuclides are usually detected using ionisation chambers. AMS provides the lowest detection limit of all mass spectrometry methods, reaching atomic abundances as low as $10^{-16}$.

If AMS is used for the detection of stable elements, it is named Trace Element AMS (TEAMS, e.g., Ott et al. 2012) or if the Cs-beam is focussed so as to provide spatial resolution, Accelerator-SIMS or Super-SIMS, which provides detection limits some orders of magnitude better than is the case for traditional dynamic SIMS.

IBA provides information on the composition and spatial distribution of chemical elements in a sample using swift ions produced by a particle accelerator with energies ranging from hundreds of $\mathrm{keV}$ up to several $\mathrm{MeV}$. The particles and/or electromagnetic radiation generated

This article is protected by copyright. All rights reserved. 
by the nuclear and atomic interactions resulting from the ion beam striking the sample are then used to deduce information about the "unknown" material. The most widely employed IBA methods are Rutherford Backscattering Spectrometry (RBS), Elastic Recoil Detection (ERD), Particle Induced X-ray Emission (PIXE), Particle Induced Gamma-Emission (PIGE), Nuclear Reaction Analysis (NRA), Scanning Transmission Ion Microscopy (STIM) and Ion Beam Induced Luminescence (IBIL)/Ionoluminescence (IL). IBA techniques offer a number of important advantages with respect to geochemical applications:

1 Compositional characterisation with good mass resolution (isotope separation is possible with some IBA techniques) and sensitivity (from 0.1 at.\% down to the $\mu \mathrm{g} / \mathrm{g}$ level)

2 Low atomic number element detection and quantification

3 Extended 2D mapping capability with high lateral resolution down to $\sim 30 \mathrm{~nm}$

4 Depth profiling resolution that can go below $1 \mathrm{~nm}$

5 The capability of covering the entire periodic table through judicious use of complimentary IBA techniques

6 Generally short acquisition times ( 10-20 min)

7 Non-destructive in nature.

Recent trends and challenges facing by IBA techniques have been reviewed by Zucchiatti and Redondo-Cubero (2014). Key advances of IBA with regards to geochemical applications are related to the implementation of new detectors for particle and electromagnetic radiation that had been developed initially for nuclear and particle physics research, permitting better characterisation of the geological materials in terms of detection limits, mapping capabilities, chemical speciation, shorter acquisition etc. For example, segmented X-ray detectors allow an enormous gain in efficiency per solid angle while providing concurrent, high spatial resolution mapping of multiple elements (Pichon et al. 2014, Ryan et al. 2013, Siddons et al. 2014). New types of calorimeter-based EDX detectors characterised by high-energy resolution (e.g., several eV energy resolution for $\mathrm{Mn}_{\alpha}$ line) come at the expense of a reduced detector surface and limited counting rates. Such energy resolution, comparable to the binding energy of electrons, is opening the way for many new applications in PIXE for solving chemical speciation issues. Parallel developments have also taken place with respect to charged particle detection: optimised light particle multi-channel detectors are providing higher counting rates, leading to improved signal-to-background ratios resulting in better detection limits. The last couple of years have also witnessed advances in terms of the control and acquisition systems of nuclear microprobe setups (Laird et al. 2013, Pichon et al. 2014).

This article is protected by copyright. All rights reserved. 
As the key element of both AMS and IBA is an accelerator with a terminal voltage between $200 \mathrm{kV}$ and $25 \mathrm{MV}$, both methods are often performed in the same laboratory. The number of available facilities and, thus easier access, continues to increase thanks to the transformation of old accelerators formerly used for fundamental research and, especially for AMS, the installation of dedicated machines. To date about 100 AMS facilities operate worldwide or are under installation. Although a few medium-sized machines have recently been developed which are capable of measuring radionuclides (e.g., ${ }^{36} \mathrm{Cl}$ and its troublesome isobars ${ }^{36} \mathrm{~S}$ ), the trend is clearly towards compact machines with accelerators having terminal voltages up to $1 \mathrm{MV}$ only. These facilities can reach excellent background levels (e.g., on the order of low $10^{-16}$ for ${ }^{10} \mathrm{Be} /{ }^{9} \mathrm{Be}$ ) and have real multi-isotope measurement capabilities (Klein et al. 2014). Thus, generally easier access and cheaper measurements can be expected in the near future.

\section{Advances in AMS ion source design}

With respect to AMS, key advances have been seen in ion source design. Also significant, using highly efficient He-stripping, Christl et al. (2014) have reported Am and the Cm analyses using the low-energy TANDY AMS facility; they demonstrated that Am- and Cmisotopes could be quasi-simultaneously measured using a ${ }^{243} \mathrm{Am}$ tracer.

Sometimes the ion source itself fortuitously accounts for the main isobar suppression (e.g.,

${ }^{14} \mathrm{~N}$ eliminated form ${ }^{14} \mathrm{C}$ investigations). Beyond the need for high and stable beam currents, ion source designs must take into consideration the minimisation of isobaric interferences, cross-contamination and memory effects. For the first application of ${ }^{39} \mathrm{Ar}$, which can be used for dating of ocean water samples, Collon et al. (2012) used a gas-filled magnet for suppressing the main isobar $\left({ }^{39} \mathrm{~K}\right)$ suppression. However, another important isobar reduction of a factor of 130 could be achieved by using a quartz liner in the plasma chamber of the electron cyclotron resonance (ECR) ion source. By combining these two strategies, detection limits of $4 \times 10^{-17}$ could be reached. Further modifications, such as replacing specific components in the ion source with ultrapure materials, unfortunately did not contribute to even lower potassium contamination levels. Generally, these kinds of investigations are not only relevant for ${ }^{39} \mathrm{Ar}$ determination, but could be relevant for the more widespread determination of ${ }^{41} \mathrm{Ca}$ where ${ }^{41} \mathrm{~K}$ is the troublesome isobar. Though, in contrast to ECR sources, for Cs-sputter ion sources the contamination of the Cs itself may well be the central issue.

In the case of volatile elements such as chlorine and iodine the key issue is crosscontamination and long-term memory (e.g., Finkel et al. 2013, Wilcken et al. 2013). Pavetich et al. (2014) modified their commercial ion source to reduce these effects, subsequently

This article is protected by copyright. All rights reserved. 
demonstrating the advantages of their new design by measuring samples of highly different stable chlorine ratios $\left({ }^{35} \mathrm{Cl} /{ }^{37} \mathrm{Cl}\right)$.

It would be major advance if carbon-containing environmental samples, such as speleothems or corals, could be directly introduced into an AMS source for direct ${ }^{14} \mathrm{C}$ measurements. Wacker et al. (2013) and Münsterer et al. (2014) have reported on a pulsed laser ablation system coupled to the gas ion source of the MICADAS system $(200 \mathrm{kV})$ for direct and continuous $\mathrm{CO}_{2}$ introduction by $\mathrm{He}$ flushing. The first results on two stalagmites are very promising, though the authors are claiming that there is scope for further technical improvements. In addition to saving time and costs as compared with conventional treatment, this new technique allows radiocarbon analyses at significantly improved spatial resolutions.

\section{Improvements in IBA detection and acquisition systems}

Pichon et al. (2014) have reported on the complete refurbishment and upgrades to the detector set-up of the AGLAE system in Paris, which have led to improved spatial resolution for this system. The new acquisition system allows better quality X-ray spectra with reduced beam currents. They have a multi-particle detection system with one Silicon Drift Detector (SDD) for PIXE analyses of the low Z elements, four filtered SDD's for higher Z (minor and trace) elements, a high purity Ge detector for PIGE, an annular detector for RBS and an IL spectrometer. The system has position stability controls and permits automated, unsupervised

operation for sequential applications. At best, large maps $(200 \mathrm{~mm} \times 200 \mathrm{~mm})$ of $(6 \mu \mathrm{m} \times$ $3 \mu \mathrm{m}$ ) pixels can be achieved. A multi-parameter system saves each event from the X-ray, $\gamma$ ray and particle detectors, time-tagged with the $\mathrm{X}$ and $\mathrm{Y}$ positions of the beam. The resulting large data sets meant that new software solutions had to be developed, including powerful event-by-event reconstruction software. The new AGLAE setup can be used for the characterisation of geological samples, mapping trace elements at concentration levels tens of $\mu \mathrm{g} \mathrm{g}^{-1}$ (Zucchiatti et al. 2014) and allowing mineral phase identification through the analysis of micro-PIXE quantitative elemental maps (Calligaro et al. 2014).

Ryan et al. (2013) and Siddons et al. (2014) have reported on upgrades to their large solidangle (1.2 sr) detector array Maia. The new data acquisition and detection system will allow capturing fine details in large area images (> $100 \mathrm{M}$ pixels) of complex mineral samples obtained at counting rates up to $10 \mathrm{M} \mathrm{s}^{-1}$. The dynamic analyses implemented in the GeoPIXE software permit the real-time display of deconvoluted elemental distribution maps. By combining the deep penetration of $\mathrm{MeV}$ ions, the wide range of take-off angles accessible to Maia and the X-rays self-absorption in the sample, it will be possible to identify particles (e.g., PGE grains) located well below mineral surfaces, providing an alternative for

This article is protected by copyright. All rights reserved. 
destructive depth profiling methods. In parallel to these detector developments, Laird et al. (2013) reported the development of a new Field Programmable Gate Array-based Data Acquisition System (DAS), integrated with both target positioning and beam transport system at CSIRO Nuclear Micro Probe. This new system supports the increasingly complex experimental configurations associated with IBA, featuring a high level of synchronisation needed for quantitative accuracy and cross-correlation. The performances of the new DAS have been demonstrated by micro-PIXE mapping of an apatite sample.

A detailed description of the High-Resolution High-Energy (HRHE) PIXE setup at CTN, Lisbon is given by Chaves et al. (2014a, b). This detection system covers a wide energy range (from below $1 \mathrm{keV}$ up to $120 \mathrm{keV}$ ) based on a CdTe detector with an efficiency plateau up to roughly $70 \mathrm{keV}$ and an X-ray Microcalorimeter Spectrometer (XMS) with energy resolution better than $15 \mathrm{eV}$ at $1.486 \mathrm{keV}\left(\mathrm{Al}-\mathrm{K}_{\alpha}\right)$ and less than $40 \mathrm{eV}$ at $10.550 \mathrm{keV}\left(\mathrm{Pb}-\mathrm{L}_{\alpha}\right)$. The CdTe detector can be used to measure the K X-ray lines of REE (instead of the usual L lines), allowing accurate quantification of REE with detection limits in the range of tens of $\mu \mathrm{g} \mathrm{g}^{-1}$. This detector arrangement extends the useful energy window for the PIXE technique to the medium and high $\mathrm{Z}$ elements. The XMS detector -- a transition edge sensor that measures the temperature rise in a $250 \mu \mathrm{m}^{3} \mathrm{Au}$ cube when hit by a single X-ray photon -- can detect X-ray lines with energies as low as $800 \mathrm{eV}$. The newly installed X-Y positioning system allows the mapping of geological samples of macroscopic dimensions up to several $\mathrm{cm}^{2}$. The capabilities of the system for the analysis of geological materials were demonstrated on a sample of $\mathrm{UO}_{2}$ with well-resolved $\mathrm{M}$ and $\mathrm{N}$ lines for uranium (XMS detector), $\mathrm{K}$ lines for uranium (CdTe detector) and by mapping medium and high $\mathrm{Z}$ elements in a metapyroxenite target including, REEs at hundreds of $\mu \mathrm{g} \mathrm{g}^{-1}$ concentration levels. This HRHE PIXE set-up raised the need for a revision of the software used to fit the PIXE spectra. The new DT2 package (Reis et al. 2014), with a user-friendly interface, includes both fitting and data handling codes.

The LIBAF detection system in Lund was recently upgraded with a segmented annular Double Sided Silicon Strip Detector (DSSSD) that can provide angular information and a large solid angle of detection. The performances of this position sensitive detector for ppscattering were tested by Borysiuk et al. (2013) who measured the $\mathrm{H}$ content in several geological samples (a $13 \mu \mathrm{m}$ thick olivine sample with $24 \mu \mathrm{g} \mathrm{g}^{-1} \mathrm{H}$ and a $10 \mu \mathrm{m}$ thick garnet with $\left.380 \mu \mathrm{g} \mathrm{g}^{-1} \mathrm{H}\right)$.

The analyses of $\mathrm{B}$ with high spatial resolution is interesting from a geochemical perspective as $\mathrm{B}$ is an important tracer for chemical cycling, having a high solubility in both aqueous fluids and silicate melts. LIBAF has made use of the $11 \mathrm{~B}(\mathrm{p}, 2 \alpha) \alpha$ nuclear resonant reaction in

This article is protected by copyright. All rights reserved. 
conjunction with the recently upgraded DSSSD that allows higher counting rates. This new set-up both increases the statistics imaging and improves the detection limits for a given acquisition time. Kristiansson et al. (2014) estimated detection limits for ${ }^{11} \mathrm{~B}$ in geological samples to be $\sim 5 \mathrm{~g} \mathrm{~g}^{-1}$, as well as documenting high-resolution maps of ${ }^{11} \mathrm{~B}$ in sillimanite.

$\mathrm{D} / \mathrm{H}$ ratios are of interest in geology as they can record information about the behaviour and mass transport of $\mathrm{H}$ during Earth's evolution. Simultaneous measurements of $\mathrm{D} / \mathrm{H}$ ratio by coincidence measurements using pp-scattering and pd-scattering or dd-scattering and dpscattering were presented by Ros et al. (2013). They employed a DSSSD detector providing both position and energy information. The judicious placement of the detector allowed the simultaneous detection of $\mathrm{H}$ and $\mathrm{D}$ coincidences. Their experiments showed that deuterons are the more suitable projectiles for the measurement of samples with low D/H ratios (no need to change the projectile while avoiding uncertainties imposed by charge normalisation). Ros et al. (2014) reported measurements on both a deuterated polyethylene reference material with $\mathrm{D} / \mathrm{H}$ ratio close to natural abundance and on a thin sample of muscovite with $4800 \mu \mathrm{g} \mathrm{g}^{-1}$ $\mathrm{H}$, showing that $\mathrm{D} / \mathrm{H}$ ratios as low as $2.0 \times 10^{-4}$ (corresponding to a $\mathrm{D}$ content of $1 \mu \mathrm{g} \mathrm{g}^{-1}$ ) can be measured. Here we note that this coincidence method has the same advantages as ppscattering (i.e., high cross-section, low background and low sample damage) (Reichart and Dollinger 2009), making it suitable for nuclear microprobe measurements of $\mathrm{D} / \mathrm{H}$ ratio in thin geological samples.

PIGE and NRA are ion beam methods that are extensively used for light element concentration and isotope ratio determinations. Photon tagged nuclear reaction analysis (pNRA) fuses PIGE and NRA into a single technique, resulting in improved detection limits for certain light elements (Kristiansson and Martinsson 1997). This method relies on time resolved $\gamma$-rays detection and the parallel charged particle detection resulting from the same nuclear reaction, correlating the arrival time of the particles in the detectors with the energy of the charged particle. Tagging the charged particle with the coincident $\gamma$-ray is a way of suppressing background, allowing the separation of interesting reactions from background interferences inherent in complex targets. Borysiuk et al. (2014) describe a new pNRA setup that benefits from advances in detector technology that have occurred over the past 20 years. Specifically, a $\mathrm{LaBr}_{3}$ scintillation detector with large $\gamma$-ray capture efficiency and a DSSSD with large solid angle coverage were used for the coincident detection of the $\gamma$-ray and charged particle resulting from the same nuclear reaction, respectively, leading to the significant reduction of the background and to the improvement of the detection limits. Thin samples of known areal concentrations were used to deduce the detection limits of the pNRA setup, ranging from $0.36\left(\mu \mathrm{g} \mathrm{cm}^{-2}\right) / \mu \mathrm{C}$ for $\mathrm{Na}$ in a $\mathrm{NaCl}$ matrix to $20\left(\mu \mathrm{g} \mathrm{cm}^{-2}\right) / \mu \mathrm{C}$ for $\mathrm{Al}$ in a high-purity $\mathrm{Al}$ target. The pNRA technique could also be applied for the analysis of isotopic

This article is protected by copyright. All rights reserved. 
ratios of light elements such as $\mathrm{B}$ and $\mathrm{O}$ in geological samples. In the near future this same group of researchers envision that $\delta^{11} \mathrm{~B}$ measurements will be possible with pNRA by combining ${ }^{10} \mathrm{~B}(\mathrm{p}, \alpha \gamma){ }^{7} \mathrm{Be}$ nuclear reaction in conjunction with NRA on ${ }^{11} \mathrm{~B}$. Such studies would be of interest for geochemistry, since the measurements of hydrous B-bearing minerals, melts and aqueous fluids show that isotopic fractionation of $\mathrm{B}$ is strongly dependent on the coordination state, as ${ }^{10} \mathrm{~B}$ has a marked preference for the tetrahedral $\mathrm{BO}_{4}$ configuration and ${ }^{11} \mathrm{~B}$ for the trigonal $\mathrm{BO}_{3}$ groups.

As part of an investigation into meteorite samples, Wunderlich et al. (2013) describe a solution for correcting the lateral dead-time based on a noise-triggered pulse generator. Silicate and oxide crystals located in the close proximity of massive $\mathrm{Fe}-\mathrm{Ni}$ grains are a challenge for IBA mapping due to the strongly varying dead-time of the detector electronics, leading to inaccurate quantitative PIXE and RBS maps on highly heterogeneous samples. Wunderlich et al. (2013) compared the number of generated and processed pulses, thereby generating a dead-time map that was then used to correct the collected spectrum on a pixelper-pixel basis. In addition, recording the dead-time map allowed the use of high beam currents providing higher counting rates and leading to a reduction in the overall measurement time. The proper functioning of this approach was validated by measurements on a synthetic test specimen as well as on a chondrule from the Açfer 094 meteorite. With such a setup, accurate quantitative maps of different chemical elements in a wide range of inhomogeneous targets could be recorded using IBA methods.

\section{IBA detector dead-time correction}

As part of an investigation into meteorite samples, Wunderlich et al. (2013) describe a setup for detector dead-time correction based on a noise-triggered pulse generator. Silicate and oxide crystals located in close proximity to massive Fe-Ni grains are a challenge for IBA methods due to the strongly varying dead-time of the detector electronics on highly heterogeneous samples; this leads to incorrect PIXE and RBS maps on a local scale. Wunderlich et al. (2013) compared the number of generated and processed pulses, thereby generating a dead-time map that could be used to correct the collected spectrum on a pixelper-pixel basis. In addition, recording the dead-time map allowed the use of high beam currents (higher counting rates), leading to a reduction in the overall measurement time. With such a setup, accurate spatial distributions of different chemical elements were recorded in a wide variety of specimens with inhomogeneous dead-time distributions (e.g., meteorite samples). The proper functioning of the technique was validated by measurements on a well characterised test material and on a chondrule from the Açfer 094 meteorite.

This article is protected by copyright. All rights reserved. 


\section{Fundamental research devoted to cosmogenic radionuclides}

Half-life determinations: Efforts continuing towards refining the half-life estimates of a variety of radionuclides that are yet to be satisfactorily constrained. An initiative reported by Kinoshita et al. (2012) has measured a significantly shorter half-life for ${ }^{146} \mathrm{Sm}$ as compared with the earlier accepted value (i.e., (67 \pm 7) Ma vs. (103 \pm 5) Ma, respectively). They have used three different nuclear beams - 50-MeV electron bremsstrahlung, 21-MeV protons and fast neutrons - to produce ${ }^{146} \mathrm{Sm}$ from ${ }^{147} \mathrm{Sm}$-enriched targets. The authors subsequently performed $\alpha$-activity measurements for establishing ${ }^{146} \mathrm{Sm} /{ }^{147} \mathrm{Sm}$ along with sophisticated AMS measurements using a gas-filled magnet to overcome the interference from ${ }^{146} \mathrm{Nd}$. The authors conclude that the new shorter half-life of ${ }^{146} \mathrm{Sm}$, which usually serves as an astrophysical and geochemical chronometer by measuring isotopic anomalies of its $\alpha$-decay daughter ${ }^{142} \mathrm{Nd}$, implies a higher initial ${ }^{146} \mathrm{Sm}$ abundance in the early solar system.

New and revisited minerals: As cosmogenic radionuclide studies continue to be dominated by only a few mineral types (e.g., quartz for ${ }^{10} \mathrm{Be}$ and ${ }^{26} \mathrm{Al}$, calcite and feldspars for ${ }^{36} \mathrm{Cl}$ ), every study focussing on other nuclide-mineral combinations is a clear highlight. Thus, the work of Zerathe et al. (2013) reinvestigating the sometimes erratic ${ }^{10} \mathrm{Be}$ results in chert merit mentioning. By using ${ }^{36} \mathrm{Cl}$ data from coexisting limestone, they could identify the well-known problems of atmospheric ${ }^{10} \mathrm{Be}$ contaminations in their chert samples. The authors demonstrated grain size dependence for contamination and also suggested a chemical cleaning method based on $\mathrm{KOH}$ treatment to reduce the contamination. This proposed cleaning protocol, however, was not fully validated as the data set consisted of two samples only; however even the limited amount of data suggests that further efforts to investigate such cleaning procedures would be work well invested. It is clear that earlier described atmospheric ${ }^{10} \mathrm{Be}$ contamination for all other mineral phases (e.g., calcite, chert, flint, but not quartz) might be a key issue leading to systematic overestimations of cosmogenic ${ }^{10} \mathrm{Be}$ concentrations and ages. That ${ }^{10} \mathrm{Be}$ contamination originating from the atmosphere must be carefully removed prior to AMS analyses could also be demonstrated by the yet preliminary but remarkable work of Granger et al. (2013b) on magnetite.

Nuclear production models: For geomorphology, tectonics, glaciology and climate applications an accurate knowledge of the so-called production rate (the production of a given cosmogenic nuclide per year and gram of target material at the specific exposure site) is an absolute prerequisite. Thus, there is on-going research to determine local production rates at independently dated calibration sites even for rather remote areas such as Greenland (Briner et al. 2012) and the Arctic (Young et al. 2013). These publications report ${ }^{10} \mathrm{Be}$ production rates of $(3.98 \pm 0.24)$ atoms $\mathrm{g}^{-1} \mathrm{a}^{-1}$ and $(3.96 \pm 0.15)$ atoms $\mathrm{g}^{-1} \mathrm{a}^{-1}$ (normalised to

This article is protected by copyright. All rights reserved. 
sea level high latitude), respectively.

To use these production rates at other nearby sites for undated events and unknown erosion processes requires scaling models that are still under constant improvement. Lifton et al. (2014) developed a flexible framework based on analytical approximations for atmospheric cosmic-ray particle spectra constraining in situ produced cosmogenic nuclides. The new model includes updated geomagnetic and solar modulation, thus taking into account temporal variability. Using their model they investigated discrepancies between earlier results, concluding that purely neutron monitor-based scaling models generally agree best with data at high geomagnetic latitudes, whereas underestimate the flux dependency on the altitude, especially at $>2000 \mathrm{~m}$. On the contrary, Lifton's model showed best constancy with the oldest model (Lal 1991) at low geomagnetic latitudes. Although scaling models continue to improve, the best advice still remains that on should use a production rate from a calibration site as "equal" as possible to the unknown site, the hope being that this will avoid any systematic offsets induced by temporal variations in primary flux and magnetic field characteristics or any changes in secondary flux due to altitude and latitude influences.

Parameters influencing the actual production of cosmogenic radionuclides near the terminus of their pathway have been addressed by two recent papers. Firstly, Dunai et al. (2014) measured thermal neutron fluxes in experimental granite piles to study the effect of hydrogen-rich covers. They simulated hydrogen-rich covers by using polyethylene and water as proxies for snow and plant matter. These authors showed a significant increase in the thermal neutron flux in an underlying rock surface. They concluded that the rock itself is the main source of the neutrons, whereas the hydrogen-rich cover behaves as a neutron reflector. Their findings are important for future applications based on such nuclides as ${ }^{3} \mathrm{He},{ }^{36} \mathrm{Cl}$ and ${ }^{41} \mathrm{Ca}$, which are sensitive to thermal neutrons fluxes. Thus, hydrogen-rich covers, especially snow, will greatly enhance production rates if target elements like ${ }^{6} \mathrm{Li},{ }^{35} \mathrm{Cl}$ and ${ }^{40} \mathrm{Ca}$ are abundant. It will be essential to consider such elevated production rates if accurate dating is to be achieved. From an alternative perspective, this new realisation might open new possibilities for the reconstruction of the size and time span of such hydrogen-rich covers for climate reconstruction, by using paired radionuclide schemes in which one system is sensitive to thermal neutron and the second (mainly high-energy particle spallation produced) cosmogenic nuclide is unaffected by thermal neutrons.

Delunel et al. (2014) addressed the problem of snow cover using a different approach. They present long-term neutron-detector monitoring data from a natural setting from July 2001 to May 2008 at seven stations located throughout the Ecrins-Pelvoux massif (French Western Alps) and its surroundings with elevations extending from 200 to $2500 \mathrm{~m}$. Their results

This article is protected by copyright. All rights reserved. 
suggest that the fast neutrons are more strongly attenuated in snow than predicted by a conventional mass-shielding approach and the attenuation length estimated from the atmosphere. For future applications the authors propose an empirical model allowing the calculation of snow-shielding correction factors as a function of snow water-equivalent values for studies using ${ }^{21} \mathrm{Ne}$-analyses in $\mathrm{Mg}$-rich and ${ }^{36} \mathrm{Cl}$-analyses in $\mathrm{K}$-rich minerals from mafic and ultramafic rocks. This study, along side that of Dunai et al. (2014), is of utmost importance for climate and geomorphology applications, where hydrogen-rich covers are omnipresent.

\section{Applications of cosmogenic radionuclides}

Fukushima, Chernobyl and nuclear bomb tests: The disastrous accident at the Fukushima Daiichi nuclear plant in March 2011 led to numerous papers investigating the release of P and ${ }^{129}$ I. Steinhauser (2014) very recently summarised most of the Fukushimastudies - including those using AMS for Pu and ${ }^{129} \mathrm{I}$. Remarkably, Miyake et al. (2012) showed that the long-lived iodine isotope ${ }^{129} \mathrm{I}$ (half-life $=15.7 \mathrm{Ma}$ ) can also be an excellent tool for reconstructing the radiation dosimetry of shorter lived ${ }^{131} \mathrm{I}$ (half-life $=8$ days) by analysing several surface soil samples collected around Fukushima for which the ${ }^{131}$ I level had yet to have been measured. In addition to Pu, Shinonaga et al. (2014) identified nonnatural uranium from Fukushima in aerosol samples at a distance of $120 \mathrm{~km}$ just a few days after the accident.

That this unwanted release will bother us for a very long time is obvious. However, the importance of on-going investigations of long-lived radionuclides is highlighted by the paper of Roux et al. (2014). They found ${ }^{36} \mathrm{Cl} / \mathrm{Cl}$ ratios in groundwater, trench soil and leaf leachates one to five orders of magnitude higher than the theoretical natural ${ }^{36} \mathrm{Cl} / \mathrm{Cl}$ ratio 28 years after the explosion of the Chernobyl nuclear facility. The authors conclude that ${ }^{36} \mathrm{Cl}$, due to its conservative property, is an extremely good tool for determining the maximal extent of the contamination plume from the trench in groundwater.

An anthropogenic radionuclide release more than 60 years ago was investigated by Lachner et al. (2014). They measured ${ }^{10} \mathrm{Be},{ }^{14} \mathrm{C}$ and ${ }^{41} \mathrm{Ca}$ concentrations in coral sand from the Bikini atoll to reconstruct the neutron flux caused by the 1954 Castle Bravo nuclear bomb explosion. ${ }^{41} \mathrm{Ca}$ from coral $\mathrm{CaCO}_{3}$ was shown to be to be the best neutron flux monitor. These authors found ${ }^{10} \mathrm{Be}$ to be of limited use due to possible atmospheric contamination, whereas high ${ }^{14} \mathrm{C}$ concentrations indicated additional production ways besides neutron capture on ${ }^{13} \mathrm{C}$, meaning that both lighter nuclides are less-suitable for retrospective dosimetry.

This article is protected by copyright. All rights reserved. 
Marine environment and oceanography: Anthropogenic ${ }^{236} \mathrm{U}$ has recently been identified as another powerful transient and extremely conservative oceanic tracer (e.g., Sakagushi et al. 2012, Winkler et al. 2012). Subsequent studies, made possible by the fact that ${ }^{236} \mathrm{U}$ can be measured at rather small (0.5 MV) AMS facilities, have confirmed this. Among these, are the first data from the North Sea (Christl et al. 2013) and the first transect of ${ }^{236} \mathrm{U}$ in the North Atlantic Ocean composed of 70 seawater samples collected from nine depth profiles (Casacuberta et al. 2014).

Geomorphology and young climate events: As mentioned in the last review (Wiedenbeck et al. 2012), Akçar et al. (2012) successfully analysed low ${ }^{10} \mathrm{Be}$ concentrations in quartz-rich boulders originating from a historic rock fall in AD 1717. However, in their latest publication (Akçar et al. 2014) about this project they had to reject their original site for calibrating the ${ }^{10} \mathrm{~B}$ system. They concluded that ${ }^{10} \mathrm{Be}$ production rates at the rock fall site could only be interpreted as an upper bound for production rates in the Massif region over the past 300 years, as it is very probably influenced by inheritance. This interpretation agrees with studies by Merchel et al. $(2013,2014)$ that also found biased results from medieval rock falls as calibration sites ${ }^{36} \mathrm{Cl}$ production, which is even more sensitive to inheritance. Of course, problems of inherited nuclides influence not only calibration site studies, but also generally all application works: the shorter the exposure, the higher is the risk of inheritanceinduced systematic age bias. However, Schimmelpfennig et al. (2014) have analysed ${ }^{10} \mathrm{Be}$ in fourteen boulders from different moraines from the Steingletscher (Central Alps, Switzerland), deducing ages of 140-580 years without any hints of being influenced by inheritance. Thus, the authors argue, ${ }^{10} \mathrm{Be}$-dating of young moraines can be a very valuable method to directly link palaeo-glacier-chronologies to historical records and present-day glacier evolution.

Glaciology and climate studies: Numerous applications of cosmogenic radionuclides to glaciology and climate research, some of them investigating contemporary climate change, have been published in the last years in highest-ranking journals. The general idea is that the reconstruction of ancient ice-sheets responses to climate perturbations might help scientists better understand current and future cryospheric responses to climate changes. Although the experimental data is of outstanding quality, the interpretations often remain debatable.

Johnson et al. (2014) analysed ${ }^{10} \mathrm{Be}$ in glacially transported rocks from the Pine Island Glacier, a major outlet of the West Antarctic Ice Sheet. They interpreted their data as recording a rapid thinning at an average rate of more than $1 \mathrm{~m}$ per year for decades in the early Holocene ( $8 \mathrm{ka}$ ago). Young et al. (2012) - with the same AMS team on board - found that during the $150 \mathrm{yr}$ long cold reversal occurring $8.2 \mathrm{ka}$ ago (proven by earlier ice core

This article is protected by copyright. All rights reserved. 
data), the colder temperatures were associated with the advance of mountain glaciers at Baffin Bay, in arctic Canada. They also ${ }^{10} \mathrm{Be}$-dated erratics from moraines. The authors concluded that during the 8.2-ka event mountain glaciers had expanded even further than at the time of the Younger Dryas cold interval (11.7-12.9 ka ago). Finally, Jomelli et al. (2014) present a new ${ }^{10} \mathrm{Be}$ moraine chronology from Colombia, showing that glaciers in the northern tropical Andes expanded to a larger extent during the Antarctic cold reversal (12.9-14.5 ka ago) than during the Younger Dryas. They even assert that during the Younger Dryas the northern tropical Andes glaciers on the contrary "retreated owing to abrupt regional warming in response to reduced precipitation and land-surface feedbacks triggered by a weakened Atlantic meridional overturning circulation", i.e., as a result of progressive warming, probably influenced by an increase in atmospheric $\mathrm{CO}_{2}$.

Bierman et al. (2014) measured atmospherically produced ${ }^{10} \mathrm{Be}, \mathrm{C}$ and $\mathrm{N}$ from soil preserved in basal ice recovered from the Summit locality in Greenland. Their very exceptional data interpretation was used to conclude that pre-glacial landscapes are preserved for long periods under continental ice sheets due to ice sheet stability through the whole Pleistocene (the past $2.7 \mathrm{Ma})$.

Jansen et al. (2014) addresses the on-going debate over the origins of inner gorges covered by glaciers through ${ }^{10} \mathrm{Be}$ exposure dating of seven gorges positioned along $\sim 500 \mathrm{~km}$ of the former Fennoscandian ice sheet margin. They interpret their data, measured as duplicates at two AMS facilities, as proof for the cutting of the gorges by channelised subglacial meltwater while being covered by ice, rejecting fluvial erosion by subaerial rivers.

A rather indirect, but interesting climate application is the work by Rybczynski et al. (2013). These authors determined ${ }^{10} \mathrm{Be}$ and ${ }^{26} \mathrm{Al}$ on five samples of coarse sand from two fossil sites for burial dating. They also reported the first evidence of a High Arctic giant camel living in a forested Arctic (identified using collagen fingerprinting), which originated in North America and dispersed to Eurasia via the Bering land bridge between Alaska and Siberia.

Larsen et al. (2014) investigated ${ }^{10} \mathrm{Be}$ and $\mathrm{Zr}$ concentrations from soils and sediments in the western Southern Alps of New Zealand, from which they deduce catchment-wide denudation rates as high as $2.5 \mathrm{~mm} \mathrm{a}^{-1}$. Such high rates support the theory that mountains from tectonically uplifting landscapes play a key role in global-scale chemical weathering, thereby influencing the global carbon cycle and climate.

Anthropology: Of course, the classical ${ }^{14} \mathrm{C}$ dating of remnants of human beings has produced thousands of remarkable results since the invention of AMS. However, the short-

This article is protected by copyright. All rights reserved. 
half-life of ${ }^{14} \mathrm{C}(\sim 5.7 \mathrm{ka})$ means this decay system is unable to address any material older than roughly $60 \mathrm{ka}$. Thus, indirect dating methods using longer-lived radionuclides are of great interest to anthropologists. One of the most active research groups in this domain is at ASTER, the French national AMS facility, which has recently reported two major breakthroughs. Firstly, Lebatard et al. (2014) could show the presence of Homo erectus (Kocabaş hominid) at the Anatolian Peninsula more than $1 \mathrm{Myr}$ ago. They performed a multidisciplinary study including sedimentological, palaeontological and palaeoanthropological observations accompanied by palaeomagnetic measurements and determinations of cosmogenic nuclide concentration for ${ }^{26} \mathrm{Al}-{ }^{10} \mathrm{Be}$-burial ages. The work led to an absolute chronological framework for the upper fossiliferous Travertine unit where the skull and fauna had been found. The age of the fossil-bearing section is, thus, very likely in the 1.1 to $1.3 \mathrm{Ma}$ range, which is in perfect agreement with the late Villafranchian age inferred for the mammalian fauna. Secondly, Vallverdú et al. (2014) investigated the arrival of the Acheulian in Europe by analysing samples from the Barranc de la Boella site (Catalonia, Spain), a lithic assemblage containing large cutting tools. Burial ages and denudation rates were calculated based on ${ }^{10} \mathrm{Be}$ and ${ }^{26} \mathrm{Al}$ concentrations in samples of sand and fine gravel. The authors showed that the Acheulian technical tradition first appeared in Europe $\sim 0.9 \mathrm{Myr}$ ago matching hominine colonisation of the subcontinent.

Based on the same cosmogenic nuclide burial dating technique, Gibbon et al. (2014) presented new radiometric age estimates of 2.19, 1.80 and $0.96 \mathrm{Ma}$ for different members of the Swartkrans Formation in South Africa and compared them with published data provided by other dating techniques. Their new dates provide the complete age range for the extinct hominin, Paranthropus robustus. Also important, this study indicates ages for both the first appearance of the genus Homo itself and for manufacturing and use of bone digging tools in southern Africa. The authors proved "some of the earliest evidence of stone tool use and large animal butchery in South Africa; and one of the earliest archaeological indications of the domestication of fire in the world" (Gibbon et al. 2014).

\section{Future perspectives for accelerator-based methods}

Not only is ${ }^{129} \mathrm{I}$ the focus of much research due to the Fukushima incident, this radionuclide seems to be increasingly targeted, especially for environmental and geological applications (e.g., review of Fan et al. 2013). As already mentioned in the introduction, even very small AMS facilities now have the capability of producing high-precision ${ }^{129} \mathrm{I}$ data. The improved situation regarding machine access has been accompanied by several studies devoted to ${ }^{129} \mathrm{I}$ advances in sample preparation using preconcentration or coprecipitation steps (Bamba et al. 2014, Mostafa et al. 2014, Zhang et al. 2013), and including protocols for chemical

This article is protected by copyright. All rights reserved. 
separation procedures that also allow for valuable speciation analysis (e.g., Schwehr et al. 2014). That chemical sample preparation is a key issues in iodine analysis was shown years ago (Roberts et al. 1997, Roberts and Caffee 2000) by performing interlaboratory comparisons that resulted in orders of magnitude variation in results. Thus, these "advanced recipes" still have to prove that they produce accurate data for several kinds of matrices and can be easy and robustly copied by other laboratories.

However, as mentioned above, a second key issue highlighted in several investigations of chlorine (Finkel et al. 2013, Pavetich et al. 2014, Wilcken et al. 2013) is the risks from crosscontamination and long-term memory in the ion source. Similar concerns apply to other volatile elements such as iodine. Therefore, advanced measurement strategies including "wasting" precious accelerator time on cleaning the ion source between samples and the use of additional quality control materials that are fit-for-purpose having variable ${ }^{129} \mathrm{I} /{ }^{127} \mathrm{I}$ ratios (Schwehr et al. 2014) need to be developed further. Such fundamental work needs to be undertaken so that robust analytical protocols will assure optimal ${ }^{129}$ I data from unknown samples. Ultimately, it should be standard practice that all normalisation standards shall be traceable to primary standards and, furthermore, quality assurance should be fostered by regular participation in round-robin exercises, which the "AMS community" should initiate and encourage. Of course, the latter is also true for all other radionuclides, not only for ${ }^{129} \mathrm{I}$, and for AMS and IBA analyses of stable elements.

\section{Advances in secondary ion mass spectrometry}

(Contribution by: M. Wiedenbeck)

\section{Overview of the SIMS method}

Secondary ion mass spectrometry (SIMS, also known as the ion probe or ion microprobe method) is a well established techniques in the geosciences stretching back into the early 1970's, with some of the earliest applications devoted to the then recently returned Lunar materials (e.g., Andersen and Hinthorne 1972). Basically, SIMS instruments employ a primary ion beam with energies up to a few tens of $\mathrm{keV}$, which is focused on the surface of a polished sample under vacuum. A small fraction of the material eroded from the sample is ionised during the so-called sputtering process and these ions are accelerated directly into a mass spectrometer for analysis. This arrangement makes possible a number of analytical strategies including: (1) trace element quantifications with limits of quantification typically in the tens up to perhaps $100 \mathrm{ng} \mathrm{g}^{-1}$, (2) isotope ratio determinations with major elements repeatabilities reaching to below $\pm 0.2 \%$ o $(1 s)$ using the most modern multi-collection

This article is protected by copyright. All rights reserved. 
instruments, and (3) depth profiling analyses where the depth resolution of a few tens of nanometres is often defined by the surface roughness of the sample. Certain instrument designs can directly image element distributions in ion microscope mode, capable of providing distribution maps with spatial resolutions of a few $\mu \mathrm{m}$ at concentrations down to $\mu \mathrm{g}$ g-1 levels. Compared with the main competing technology of laser ablation sampling, SIMS has 1 to 2 orders of magnitude better sensitivity, meaning that total sampling masses for SIMS analyses are typically a few ng or less. Significant drawbacks of SIMS compared with LA-ICP-MS determinations are the ion probe's need for very carefully prepared samples, the need to matrix-match the calibration material to the sample of interest and, in general, a higher initial investment cost.

Over the decades a spectrum of SIMS instruments has become commercially available, with each design having its specific advantages in terms of for example spatial resolution, isotopic ratio uncertainty or technical complexity. An early review of the SIMS method as applied to geochemical studies is provided by Hinton (1995). A good overview of both modern SIMS instrumentation and SIMS applications to geochemical research is provided by Fayek (2009). A review specific to the Cameca NanoSIMS instrument is provided by Hoppe et al. (2013), with a particular focus on applications in cosmochemistry and biological geochemistry. World-wide there are currently some four dozen SIMS facilities active in the field of geochemistry.

\section{Milestone SIMS applications in geochemistry since 2012}

High precision isotope studies: Large geometry SIMS instruments, representing topend hardware for this application, continue to focus much of their work on high precision isotope determinations. State-of-the-art instrumentation can today produce data sets with repeatabilities routinely better than $\pm 0.2 \%$ o $(1 s)$ for major elements from a variety of materials. Common applications include $\delta^{18} \mathrm{O}$ determinations on silicates and $\delta^{34} \mathrm{~S}$ determinations on sulfides, both of which can be conducted on $\sim 200$ pg total sampling masses equivalent to a spatial resolution of $\leq 10 \mu \mathrm{m}$. A recent such report by Whitehouse (2013) devoted to sulphur isotope ratio determinations on sulphides is illustrative of both current technological capabilities as well as analytical issues related to investigating minor isotopes such as ${ }^{36} \mathrm{~S}$. For this work Whitehouse (2013) used a Cameca 1280 instrument operating in static multi-collector mode both without and with an NMR magnetic field controller. A constraint on this study was the goal of marinating a beam diameter of $10 \mu \mathrm{m}-$ equivalent to a $2.5 \mathrm{nA}^{133} \mathrm{Cs}^{+}$primary beam current, as had been reported in earlier sulphur isotope ratio studies. The multi-collector configuration included three Faraday cups and a narrow format Hamamatsu electron multiplier positioned at the L'2, L1, C and H2 positions

This article is protected by copyright. All rights reserved. 
for ${ }^{32} \mathrm{~S},{ }^{33} \mathrm{~S},{ }^{34} \mathrm{~S}$ and ${ }^{36} \mathrm{~S}$, respectively. For the ${ }^{32} \mathrm{~S}$ mass position a $10^{10} \Omega$ resistor was used whereas the ${ }^{33} \mathrm{~S}$ and ${ }^{34} \mathrm{~S}$ signals used a $10^{11} \Omega$ resistor (see also section above devoted to TIMS amplifiers). The mass spectrometer was operated at a mass resolution of $\mathrm{M} / \Delta \mathrm{M} \approx$ 4860 , assuring an adequate separation of the ${ }^{32} \mathrm{~S}^{1} \mathrm{H}$ signal from ${ }^{33} \mathrm{~S}$ mass position. This set up resulted in a typical count rate of $1 \times 10^{9} \mathrm{cps}$ on the ${ }^{32} \mathrm{~S}$ mass position and a typical data collection time of $64 \mathrm{~s}$.

A limitation on the data quality was that within-run drift towards lighter ${ }^{34} \mathrm{~S} /{ }^{32} \mathrm{~S}$ values was often observed, sometimes resulting in as much as $2 \%$ decrease in this value during the roughly 1 minute of data acquisition (Figure 4), leading to an overall within-run uncertainty of up to $\pm 0.4 \%$ o in $\delta^{34} \mathrm{~S}$. Fortunately, the rate of drift tended to remain fairly constant throughout a given session such that the "between-run" repeatability was not significantly degraded. Whitehouse (2013) also reports that the rate of drift within single analyses clearly differed between phases, with pyrrhotite showing roughly double the within-run drift of pyrite measured during the same analytical session. The author's preferred explanation for this phenomenon is that it results from changes in fractionation during sample sputtering, and that such changes are influenced by the primary beam geometry when it reaches the sample surface. Between-run drift in $\delta^{34} \mathrm{~S}$ was not observed during this study. Such "down-hole" fractionation shifts during analyses are known for SIMS inter-element ratio determinations (see also SIMS geochronology section, below) and these can be suppressed by using a small primary beam raster during analyses. The use of beam rastering is now an area of active development in SIMS isotope ratio determinations.

$<<$ Figure 4 approximately here $>>$

Also contained in Whitehouse's (2013) study is the first detailed description of the determination of $\Delta^{36} \mathrm{~S}$ by SIMS. This topic is particularly challenging, as it requires the use of an electron multiplier for the ${ }^{36} \mathrm{~S}$ mass station, which has an ion current some 5,000 times lower than that on the associated ${ }^{32} \mathrm{~S}$ mass station. This study reports that the Hamamatsu ion multiplier system shows a significant drift in sensitivity, where the evolution of detector efficiency is a function of the total ion dose applied to the first dynode. Using a method similar to that for the FC/FC determinations, within-run drift in the measured ${ }^{36} \mathrm{~S} /{ }^{32} \mathrm{~S}$ ratio was corrected. Between-run drift in the ${ }^{36} \mathrm{~S} /{ }^{32} \mathrm{~S}$ ratio, ranging between -0.02 and $-0.36 \%$ per analysis, could be corrected manually through a linear interpolation through the data points run on the reference materials. An alternative strategy using a software tool provided by the manufacture, the so-called pulse height distribution algorithm, which assesses the characteristics of the pulses delivered by the counter to the EM pre-amplifier, was also used. These various correction strategies revealed only a partial correlation between EM sensitivity

This article is protected by copyright. All rights reserved. 
and integrated dose; the repeatability of the ${ }^{36} \mathrm{~S} /{ }^{32} \mathrm{~S}$ ratio fell in the range of \pm 0.2 to $\pm 0.7 \%$ o $(1 s)$ for the 18 analytical session in which ${ }^{36} \mathrm{~S}$ was determined. Nonetheless, this work demonstrates that with Cameca 1280 instruments $\Delta^{36} \mathrm{~S}$ can be acquired with a data quality sufficient to permit geological process to be discerned (Figure 4). This author foresees further improvements in data quality for EM/FC determinations from either exchanging the small format Hamamatsu detectors for more robust detector designs or from further developments in software correction algorithms.

The overall between-run repeatability on 1254 measurements on the Ruttan pyrite (Crowe and Vaughan 1996, Ruttan pyrite $\delta^{34} \mathrm{~S}_{\mathrm{CDT}}=+1.2 \pm 0.1 \%$ ) reported by Whitehouse (2013) of $\pm 0.35 \% \circ$ and $\pm 0.22 \% \circ(2 s)$ for $\delta^{34} \mathrm{~S}$ and $\Delta^{33} \mathrm{~S}$, respectively, clearly shows that modern SIMS instrumentation is capable of producing isotope ratio determinations on major elements with individual uncertainties of better than $\pm 0.2 \%$ o $(1 s)$ on sampling sizes on the order of a few hundred picograms and as measured by repeatability of numerous determinations on supposedly homogeneous materials. This observation leads to three further conclusions, namely (1) SIMS instrument technology is no longer the limiting factor in defining total analytical uncertainty, but rather the quality of the RM characterisation is now becoming the dominant contributor, (2) the quality of isotope data from well equipped and experienced SIMS facility is such that direct comparison between labs at stated uncertainty levels can only be meaningfully made if the same calibrant is used or if the chain of traceability is kept very short, and (3) refined methods for homogeneity testing are needed so that this component can be eliminated from the total uncertainty budget assigned to micro-analytical RMs. Finally, in the case of isotopic systems requiring corrections for major element compositions (see SIMS matrix effects section below) there is little to be gained by further improvements in instrument data quality unless both the matrix effect correction models and the RMs upon which they are based are improve.

Uranium-lead geochronology: As has been the case for several decades, U-Th- $\mathrm{Pb}$ dating of geological materials, and in particular of zircon, continues to be a major focus of many SIMS investigations. Efforts to improve both data quality as well as the software for converting raw SIMS data into geologically meaningful results are on-going. One possible trend that might be emerging are efforts by some SIMS facilities to recover data at sampling scales significantly smaller than the 20 to $30 \mu \mathrm{m}$ diameter pits, which have long been the norm for SIMS zircon analyses. One strategy towards finer sampling dimensions has employed NanoSIMS instrumentation; such development work has been reported by Yang et al. (2012), who investigated both the zircon and baddeleyite systems. In the case of zircon, these authors used $\mathrm{a} \sim 500 \mathrm{pA}$ Gaussian ${ }^{16} \mathrm{O}^{-}$primary probe which they could focus to a $<2$ $\mu \mathrm{m}$ diameter beam at the sample surface with a total time of $\sim 30 \mathrm{~min}$ per analysis. The

This article is protected by copyright. All rights reserved. 
primary beam was rastered over a $3 \mu \mathrm{m} \times 3 \mu \mathrm{m}$ area during the analyses in order to suppress variations in $\mathrm{U}-\mathrm{Pb}$ fractionation during the course of single analyses, yielding an overall spatial resolution for their analyses of around $5 \mu \mathrm{m}$. Compared with "conventional" SIMS zircon analyses using large geometry instruments, such as the Cameca 1280 or SHRIMP instruments, Yang et al. (2012) found their NanoSIMS 50L approach benefitted from a higher primary beam density. Disadvantages of using the NanoSIMS were a loss in sensitivity relative to 1280 -class instruments due to the absence of oxygen flooding in the sample chamber and the inability to do multi-collection on the Pb-spectrum because NanoSIMS instruments do not provide sufficient dispersion between single Dalton mass differences at such heavy masses. Yang et al. (2012) also report operating at a mass resolution of 5000 at mass ${ }^{206} \mathrm{~Pb}$, but a mass scan showed extensive overlap between this peak and the ${ }^{178} \mathrm{Hf}^{28} \mathrm{Si}+$ peak located $\mathrm{M} / \Delta \mathrm{M}=3830$ below the ${ }^{206} \mathrm{~Pb}$ mass position. This discrepancy may be due to the different means of defining "mass resolution" vs. "mass resolving power" as used by the NanoSIMS community. Another issue perhaps worth considering is why a ${ }^{16} \mathrm{O}^{-}$primary beam was preferred over a ${ }^{16} \mathrm{O}_{2}{ }^{-}$as would be used by large geometry instruments due to the higher sensitivity it offers for $\mathrm{Pb}-$ is it a matter that ${ }^{16} \mathrm{O}^{-}$ provides a small beam diameter for a given primary beam current? One final observation is that no attempt was made to include ${ }^{232} \mathrm{Th} /{ }^{208} \mathrm{~Pb}$ decay system in these analyses, which could have been done without lengthening the total analysis time. These observations aside, this paper contributes valuable understanding of the capabilities of NanoSIMS instrumentation with regards to $\mathrm{U}-\mathrm{Pb}$ dating, an approach that holds much promise when only small sample domains are available to the analyst.

A second approach towards fine scale U-Pb analyses in zircon employing a Cameca 1280 instrument has been described by Liu et al. (2011), and this methodology has now been used for the dating of very grained zircons recovered from five eucrite meteorites (Zhou et al. 2013). These latter workers employed a 100 to 200 pA, Gaussian primary beam that could be focused to a $<5 \mu \mathrm{m}$ diameter at the sample surface. Oxygen flooding was used in order to improve sensitivity for the $\mathrm{Pb}$ isotopes by roughly a factor of $\sim 2$ above that obtained under high vacuum conditions in the secondary ion source. Zhou et al. (2013) actually report on two different strategies for obtaining dates from the small zircons inherent to their samples. For determining ${ }^{207} \mathrm{~Pb} /{ }^{206} \mathrm{~Pb}$ ages Zhou et al. (2013) used a static, multi-collection set-up which required $\sim 15$ min of data acquisition, subdivided into eighty cycles. Mass resolution for the multi-collection system was $\sim 8000$ at 50\% peak height. Four electron multipliers were used for the ${ }^{204} \mathrm{~Pb}^{+},{ }^{206} \mathrm{~Pb}^{+},{ }^{207} \mathrm{~Pb}^{+}$and ${ }^{90} \mathrm{Zr}_{2}{ }^{16} \mathrm{O}_{2}{ }^{+}$mass stations and daily calibrations of instrumental fractionation and any relative drift on the four detectors employed Phalaborwa baddeleyite, which the authors knew to behave similarly to zircon in term of isotopic matrix effects. Corrections for common $\mathrm{Pb}$ were based on the observed ${ }^{204} \mathrm{~Pb}^{+}$signal. Despite the

This article is protected by copyright. All rights reserved. 
very small sampling volume, using this strategy it was possible for these authors to obtain ${ }^{207} \mathrm{~Pb} /{ }^{206} \mathrm{~Pb}$ age uncertainties of around $\pm 20 \mathrm{Ma}(1 s)$ on $4.5 \mathrm{Ga}$ old zircons.

Zhou et al. (2013) also presented a second method for small scale geochronology based on U$\mathrm{Pb}$ dating operating in mono-collection mode. Fifteen cycles of the peak stepping sequence ${ }^{180} \mathrm{Hf}^{16} \mathrm{O}^{+}$(used for correcting for magnetic field drift), ${ }^{92} \mathrm{Zr}_{2}{ }^{16} \mathrm{O}^{+},{ }^{204} \mathrm{~Pb}^{+},{ }^{206} \mathrm{~Pb}^{+},{ }^{207} \mathrm{~Pb}^{+}$, ${ }^{208} \mathrm{~Pb}^{+},{ }^{238} \mathrm{U}^{+}$, and ${ }^{238} \mathrm{U}^{16} \mathrm{O}_{2}{ }^{+}$were conducted for each analysis, leading to a total analysis time of $\sim 15$ min. Mass resolution in multi-collector mode was set at 7000 at $50 \%$ of peak height. Correction for common $\mathrm{Pb}$ made use of the observed ${ }^{204} \mathrm{~Pb} /{ }^{206} \mathrm{~Pb}$ for each individual analysis and the U/Pb calibration was based on the M257 zircon reference material (Nasdala et al. 2008). Using their mono-collection protocol, Zhou et al. (2013) reported typical analytical uncertainties for ${ }^{207} \mathrm{~Pb} /{ }^{206} \mathrm{~Pb}$ ages of $\pm 25 \mathrm{Ma}$ and for ${ }^{206} \mathrm{~Pb} /{ }^{238} \mathrm{U}$ ages of $\pm 100 \mathrm{Ma}(1 s)$ on their $\sim 4.5 \mathrm{Ga}$ old zircon grains. Some of the samples yielded analyses that are significantly reverse discordant.

These examples document the possibility and the limitations at greatly reduced sampling volumes for SIMS-based zircon geochronology. Clearly the reduction of the total mass consumed down to the low picogram level is associated with poorer data statistics; nonetheless, these studies demonstrate that useful data can be recovered even when one is faced with grain sized of less than $10 \mu \mathrm{m}$. It seems probable that further advances will be made in the realm of fine-scale dating in the coming years. Improvements in our understanding of the sputtering process as well as refinements to calibration protocols may likely prove key for major advances in this field.

Matrix effects: One of the greatest challenges facing SIMS as a tool in the geosciences is the common necessity to correct for the presence of "matrix effects", whereby the relative sputtering yields of distinct elements or of individual isotopes is significantly influenced by the chemical composition of the material being analysed. (Matrix effects resulting from the cryptographic orientation in certain mineral/trace element systems have been reported, but such phenomena are not discussed here.) For those mineral phases with little or no variation in their major element contents (e.g., quartz, zircon) there is generally little need to worry about the issue of matrix effects as long as the sample materials is the same phase as the RM used for calibrating the SIMS instrument. When dealing with samples with extended compositional ranges, there are basically two means for correcting for the influence of major element composition on the sputtering of geological materials: (1) by using a "closely matched" RM for each material being analysed, or (2) using a spectrum of RM which cover the most of the compositional space of the given phase and then to develop a mathematical correction for interpolating between RM compositions to define a correction

This article is protected by copyright. All rights reserved. 
factor for each individual location being analysed from the sample material. The first of these strategies suffers from two problems. Firstly, one must define the concept of "closely matched" such that any differences between the calibration and sample material are small enough that variations in sputtering behaviour are negligible. Secondly, one is compelled to produce a very large number of well characterised RMs in order to cover all possible compositions within a given mineral/isotope system. Thus, the second strategy, of using a mathematical model to interpolate a correction coefficient, is generally the more realistic approach - albeit this comes with the need to propagate any analytical uncertainties introduced by the selected correction scheme.

The determination of $\delta^{18} \mathrm{O}$ in natural glasses, and in particular in melt inclusions, can provide key information for understanding magmatic systems. As SIMS is uniquely capable of determining the ${ }^{18} \mathrm{O} /{ }^{16} \mathrm{O}$ ratio at the microanalytical scale, it is essential that the effect of chemical composition on the overall instrumental mass fractionation (IMF) should be understood. The contribution by Hartley et al. (2012) provides valuable guidance in this regard. These authors investigated a suite of glass RMs that had either been characterised earlier for their $\delta^{18} \mathrm{O}$ compositions or that had been measured as part of this study. Their twelve glass RMs spanned a compositional range covering from basaltic thorough rhyolitic, all of which had well determined major element compositions. This study found variability in the IMF of up to $5.3 \%$ where, in general, the more basic materials measure a more positive IMF than was the case for more silica-rich compositions. These authors evaluated a variety of corrections schemes, including both univariate and multivariate approaches based on the chemical compositions as well as assessing correlations between other parameters such as sputtering rate and material density. Hartley et al. (2012) found strong correlations in a number of the corrections schemes that they investigated. A multivariate approach using five elements ( $\mathrm{Fe}, \mathrm{Mg}, \mathrm{Ca}, \mathrm{Na}$ and $\mathrm{K}$ ) was found to give the best correlation between composition and IMF $\left(\mathrm{R}^{2} \approx 0.981\right)$ for intermediate compositions, however this approach was found to yield poor perditions of IMF when investigating materials at the ends of the compositional range. These authors ultimately recommended the use of the univariate parameter of the $\mathrm{SiO}_{2}$ content of the sample in order to correct for composition induced variations in IMF, for which the obtained $\mathrm{R}^{2} \approx 0.962$. Using the $\mathrm{SiO}_{2}$ content alone, they found that $75 \%$ of the $\mathrm{RM}$ results were within $\pm 0.2 \%$ of the values predicted by their model and that $95 \%$ were within $\pm 0.4 \%$. This is a fairly encouraging result, as it implies that such a matrix correction is not a major source of additional uncertainty. For many SIMS determined $\delta^{18} \mathrm{O}$ value the published uncertainties are on the order of $\pm 0.2 \%$ ( $1 s)$; adding in quadrature an additional uncertainty of $\sim 0.2 \%$ o would result in a total analytical uncertainty of circa $\pm 0.3 \%$ o, which still might be of sufficient quality to address important geochemical topics. Clearly, advancing the overall data quality in such cases where matrix effects have an impact will require improving both

This article is protected by copyright. All rights reserved. 
the robustness of the correction models as well as the assigned uncertainties of the myriad of materials upon which such models are based. New measurement methods, for example major improvement in ion yields or using heavy molecular ions, which are less sensitive to matrix chemistry, might also help improve the situation. Finally, here we make a few additional observations on further work that might already be undertaken to advance such IMF correction schemes. Firstly, the role of using a small beam raster during the analyses needs to be clarified. In Hartley et al. (2012) it was not clear whether Köhler illumination was used on the primary beam or whether a small raster was employed during the analyses. Such a strategy might well change the slope of the relationship between $\mathrm{SiO}_{2}$ content and IMF. Secondly, it would seem plausible to determine the ${ }^{16} \mathrm{O}^{-} /{ }^{28} \mathrm{Si}^{-}$or the ${ }^{16} \mathrm{O}^{-} /{ }^{28} \mathrm{Si}^{2-}$ ratio so as to obviate the need for determining the absolute $\mathrm{SiO}_{2}$ content of the sample material in advance, though this would prevent the use of the NMR magnetic field probe on Cameca large geometry instruments.

A second noteworthy investigation into matrix corrections for SIMS isotope analyses is reported by Ickert and Stern (2013); they investigated the nature of oxygen isotope fractionation affecting $\delta^{18} \mathrm{O}$ determinations on garnet. These authors provide a detailed report on the nature and stability of the IMF value they observed during $\delta^{18} \mathrm{O}$ garnet analyses using their Cameca 1280 instrument; particularly valuable is their detailed portrait of all uncertainty propagation aspects of their correction model. Ickert and Stern (2013) employed 17 garnet materials in their study. In addition to assessing the impact that variations in major element composition have on the overall IMF, they also investigated the role of varying specific parameter of the secondary ion optics such as system magnification and the width of the energy band-pass. The key result of their study is that $\mathrm{Ca}-\mathrm{Mg}-\mathrm{Fe}$ rich garnets show a systematic variation in their IMF, which correlates strongly with the $\mathrm{Ca} /\left(\mathrm{Mg}+\mathrm{Fe}^{2+}+\mathrm{Ca}\right)$ atomic ratios. Using a quadratic best-fit relationship the correction was found to be reliable to $\pm 0.2 \%$ at the $95 \%$ confidence interval from across the compositional spectrum investigated. This level of reliability is similar to that possible for glass analyses as discussed above, suggesting that $\delta^{18} \mathrm{O}$ determinations on garnet can be achieved using a fairly straightforward matrix correction. Likewise, as is the case for geological glasses, the need for a matrix correction in garnet will introduce an additional uncertainty source similar to that derived from analytical repeatability. A limitation of the approach described by Ickert and Stern (2013) is that the quadratic relationship between IMF and major element composition could accommodate neither Cr-rich nor Mn-rich garnets; these end members remain a subject for future studies.

This article is protected by copyright. All rights reserved. 


\section{The focused ion beam method in the geosciences}

(Contribution by: Luiz F.G. Morales)

The focused ion beam (FIB) technique was initially developed in the 1980s by the semiconductor industry and has been widely employed since to patch and/or modify semiconductors and even $\mu \mathrm{m}$-scale cooling systems. It was only in the 2000s, however, that such techniques were first used in the Earth sciences, initially limited to the preparation of in situ transmission electron microscopy foils (e.g., Wirth 2009). More recently, with the advent of so-called "Dual Beam ${ }^{\circledR}$ " machines, geoscientists realised that a focused ion beam integrated with the imaging capabilities of the electron source has vastly broader utility within the geosciences, and in particular for 3D imaging and nanotomography. A Dual Beam® machine essentially consists of a vertical electron column, as in a standard scanning electron microscope (SEM), together with a tilted ion beam column. In addition, a number of different accessories and detectors are normally present in these machines, such as micromanipulators and gas injection needles, as well as energy dispersive X-ray spectrometers (EDS) and electron backscattered diffraction (EBSD) detectors (Figure 5A).

FIB optics resembles that of a SEM, but where the later uses a focused beam of electron to generate images, the former uses a finely focused beam of ions. Most FIB instruments in geoscience departments use a liquid-metal ion source (LMIS) normally based on gallium, although gold, iridium and lighter elements can also be used for similar purposes. The LMIS reservoir is heated to near evaporation causing the liquid metal to flow to the tip of a very sharp, heat resistant tungsten needle. The electric field at the tip of this needle ( $2 \mathrm{~nm}$ radius) induces field emission ionisation of the metal atoms. At low beam currents, the interaction of the incident ions on the target material produces secondary electron emission that can be used for imaging, similarly to the process in a SEM. However, if the beam current is high enough, the incident ions (due to their mass) are capable of sputtering atoms from the target material at the atomic scale. Three-dimensional imaging with FIB/SEM is achieved by repetitively sputtering material from the target with the ion beam and then imaging the region of interest with the electron beam between each step of sputtering; the amount of material removed by sputtering before the next image acquisition can be controlled by the user. For a representative volume at the micrometre scale a variable thickness between 50 to $100 \mathrm{~nm}$ of material is typically removed during a given step, where the optimal spacing of the SEM

This article is protected by copyright. All rights reserved. 
imaging planes depends on the grain sizes and the level of detail required for the given investigation. A detailed description of sample preparation and key setup parameters for successful analyses of most of geological materials is given by Wirth and Morales (2012).

$<<$ Figure 5 approximately here; colour $>>$

\section{FIB 3D microstructural characterisation of geomaterials}

Pore space geometry: Although FIB investigations of geological materials have been reported for some time, sample preparation for TEM studies have been its dominant application. However, in the last two years fundamental 3D microstructural investigations using the nanotomography method, coupling FIB and SEM, have been carried out both on fine-grained lithologies (e.g., shales) and on partially-melted rocks. In the last few years there has been an increasing focus on shales, with research foci on radioactive waste disposal, the production of hydrocarbon gas and $\mathrm{CO}_{2}$ sequestration. In all such cases the common goal is a complete characterisation of the geometry of the intergranular pore space in clay-rock formations. Due to inherent mechanical anisotropies and very small grain sizes, shales are highly complex rocks to prepare; it is exceedingly difficult to polish thin-sections to the level where nanoporosity can be well-defined. Keller et al. (2013) reported a fundamental study on the application of FIB technique (couple with other methods not discussed here) in shales, including a detailed study of a sample of Opalinus Clay. Due to its favourable properties for the deep disposal of radioactive wastes, the Opalinus Clay of Switzerland is a well-studied shale example: it has an extremely low permeability, is practically watertight and has selfsealing properties. In their contribution, Keller et al. (2013) have used a multi-scale approach in order to achieve a highly detailed three-dimensional characterisation of the porosity and phase distribution at variable scales. They tested several tomographic methods, spanning from the nanometre to millimetre-scale resolutions. One important aspect of this study is the impact of increasing resolution in tomography methods and the inherent decrease in the total sample volume. Because shales are very heterogeneous in terms of phase distribution and because the sample size analysed by the FIB is rather small (maximum dimensions of a few tens of $\mu \mathrm{m}$ ), the authors performed standard SEM analyses in broad ion beam milled surfaces prior the FIB nanotomography. This allowed the 2D characterisation of the microstructures enabling a better targeting of sites for the 3D methods. The authors chose two sites with contrasting degrees of compaction, which they document at the nanotomographic scale. Their

This article is protected by copyright. All rights reserved. 
work indicated that porosity ranges between 2 and 3\%, which is significantly lower than the porosity determined via lower resolution methods $(\sim 10 \%)$. Even with the sample in a position not favourable for backscatter imaging (as the sample is tilted $52^{\circ}$ from the standard parallel position with relation to the backscattered detector), the authors were capable of distinguishing different phases such as carbonates, quartz, large clay crystals and different organic materials within the clay matrix. The segmentation of the individual images acquired during the nanotomography via FIB also allowed the authors to model the pore path orientation distribution and pore geometry (Figure 5B). The pore path density distribution was shown to have a preferred orientation parallel to the bedding, whereas geometrical convolutedness of pore paths was significantly higher in the direction normal to bedding. Using the FIB method Keller et al. (2013) demonstrated that nanopore might not represent the main transport pathways in this Opalinus Clay, despite the fact that the nanopores between the clay laths represent a large fraction of the general porosity in this lithology. Furthermore, Keller et al. (2013) demonstrated that the coarser porosity observed in the FIB nanotomography is not interconnected and that porosity is concentrated along contacts between clay minerals and the other phases. Together these features indicate strong pore connectivity anisotropy, with higher values parallel to the bedding. Equally significant, it was shown that a significant fraction of the porosity is not interconnected, which would certainly influence gas extraction from such rocks. One additional aspect of this paper is that the authors clearly show that an up-scaling of FIB observations is not straightforward due to the very small observational scale; it may be necessary to use other tomography techniques to assure that the observations from the nanoscale are correctly extrapolated to the $\mu \mathrm{m}$ and $\mathrm{mm}$ scales.

Melt distribution: A second contribution making use of the FIB nanotomography methods was presented in by Garapic et al. (2013), who made extensive use of this technique for the 3D characterisation of melt distribution in partially molten mantle rocks. The dynamics of such rocks is directly related to the amount of melt retained in the solid matrix, affecting both physical (e.g., buoyancy, seismic velocities) and chemical (e.g., trace elements and isotopic) properties of the material. Garapic et al. (2013) performed experiments on olivine aggregates containing 2 and $4 \%$ of basaltic melt using a piston cylinder apparatus. The samples were then polished using only colloidal silica, thereby avoiding physical damage of the surfaces, and then analysed via FIB. Such melt-containing samples are very tricky to prepare for two reasons. The first is that because the quenched melt (a glass) is softer than the

This article is protected by copyright. All rights reserved. 
solid matrix it is preferentially removed during polishing, introducing topographic effects onto the sample surface. Hence, the electron illumination during oblique imaging is not homogeneous for both the melt and the solid matrix part, particularly near crystal-glass interfaces. This may cause serious problems during image segmentation (the statistical evaluation whereby a digital image is partitioned into multiple sets of pixels), hindering an accurate determination of 3D melt volumes. Secondly, some experiment-produced melts types can be very reactive such that they are prone to damage and charging during focused ion beam sputtering, potentially resulting in artefacts related to image drift. A careful tuning of both the ion and electron sources (by means of changing accelerating voltages and beam currents) is therefore essential for successful tomography studies in melt-rich rocks. In order to convert the typical grey-scale images from the SEM to binary images Garapic et al. (2013) performed a hand digitisation of the melt-solid interfaces prior to processing. FIB nanotomography clearly showed that the thin films separating neighbouring grains could be traced in 3D. These films were found to persist over distances of more than $10 \mu \mathrm{m}$, thereby demonstrating that such features are not simply triple-junction melt pockets, which are on the order of $1 \mu \mathrm{m}$ in diameter. Rather, such melt accumulations were seen to form thin films separating olivine grains, where such films can be classified as wetted two-grain boundaries. Such wetted two-grain boundaries exist in aggregates with dihedral angles $>0^{\circ}$, and are apparently a dynamic feature resulting from grain growth driven by surface energy reduction. One minor drawback encountered in Garapic et al.'s (2013) work was the fact that, due to the relative coarse olivine grains, only a few grains could be visualised at once; for this reason the "volume" of wetted grains imaged was rather limited.

These papers by Keller et al. (2013) and Garapic et al. (2013) show the strong potential of the FIB nanotomography for the microstructural quantification of fine-grained rocks having complex mineralogies for characterising melt distributions in partially molten rocks. These examples also show clearly that this technique can be handicapped in terms of dimensional volumes that can be analysed, mostly because the sputtering process at low beam currents coupled with high-resolution imaging is very time consuming. To give an idea, FIB nanotomography of a $25 \times 25 \times 15 \mu \mathrm{m}$ box, with $100 \mathrm{~nm}$ thick slices being removed at each step prior imaging, takes about $10 \mathrm{hr}$. Nonetheless, the level of detail that can be attained is impressive, and characterisation of nanoscale porosity and mineralogy in three dimensions is now possible in almost any sort of geomaterial.

This article is protected by copyright. All rights reserved. 


\section{Outlook for focused ion beam techniques in the geosciences}

Thanks to "Dual Beam®" equipment, geoscientists now have the ability to combine both focused ion and the electron beam sources, providing access to both $3 \mathrm{D}$ compositional and crystallographic information within micrometre-sized volumes. 3D chemical analyses using a SEM/FIB system are normally obtained by quantifying the excited X-ray photons with EDX. The acquisition of a 3D chemical composition map follows the same procedure as with the nanotomography, with an additional step that a full EDX map from the frontal surface of the target is acquired between each sputtering step with the FIB. This approach can be particularly useful in those cases where the phases present in a rock specimen do not generate enough contrast in the secondary or back-scattered electron image. As an example, quartz, calcite and feldspars may appear with similar grey levels in SE/BSE imaging, and it is virtually impossible to separate the phases by colour contrast. In such cases the chemical compositional mapping via EDX signal can be used to uniquely identify phases. The 3D resolution will depend on the milling resolution of the focused ion beam and the EDX analyses will depend on the accelerating voltage of the electron beam and on the composition of the target material to be analysed. Because the interaction between the electron beam and sputtered surface occur at an angle, the depth of interaction and emission of X-rays in these conditions is lower, commonly on the order of few 100's nanometres. One drawback of this method is that it requires very stable SEM imaging, free of electrical charging and imaging drift. Because most geological materials are non-conductive, this can be an issue that may hinder successful analyses. Furthermore, because a complete BSE or SE image has to be acquired and one full EDX map has to be generated from the frontal surface of the studied volume after each sputtering step, this procedure is very time consuming, limiting its application to carefully selected small regions in fine-grained rocks. Future developments addressing the challenges of sample charging and image stability while increasing the rate at which chemical maps can be acquired would represent significant advances in the field of FIB-based imaging. A better integration of FIB-based imaging methods with finer-scale methods (e.g., SIMS, atom probe) would also mark a significant advance.

This article is protected by copyright. All rights reserved. 


\section{Electron backscatter diffraction in the geosciences}

(Contribution by: Luiz F.G. Morales)

Electron backscatter diffraction (EBSD) analysis is a microstructural-crystallographic technique normally conducted in a scanning electron microscope; EBSD can be used to determine the crystal orientation of virtually any crystalline material at the $\mu \mathrm{m}$-scale. This is often the method of choice for crystal orientation determinations, grain boundary mapping, intra-crystalline defect characterisation, phase identification and micro-strain mapping studies. As is the case for many of the analytical techniques used in geosciences, EBSD started as a tool for material sciences, and it has now become the most widely used method for determining the crystallographic orientations of minerals and rocks. In the EBSD technique an ultra-polished crystalline specimen is placed inside the SEM chamber at a $70^{\circ}$ angle from the horizontal, facing towards the diffraction detector. The detector, which consist of a phosphor screen combined with a low luminosity CCD camera that together image the diffraction patterns, is position in the specimen chamber at $\sim 90^{\circ}$ to the SEM pole piece. In this configuration, some of the electrons that enter the sample will be backscattered and may continue on to escape from the sample. The probe diameter of the incident electron beam is typically one to tens of nm, which is roughly one order of magnitude larger than the $\AA$-scale intra-crystalline spacing typical in minerals. Because of their wave-like properties, the escaping electrons undergo Bragg diffraction by all the intra-crystalline planes at the sample target point, and these diffracted electrons form a characteristic pattern displaying different Kikuchi bands, each of which corresponds to the projection of a diffracting lattice plane. Through comparison of the observed Kikuchi pattern with all possible patterns for a given (known) phase it is possible to index the patterns by Miller indices to determine the orientation of the target domain in 3D space with good precision. All the theoretical details are beyond the scope of this review, for which the reader is referred to publications by Engler and Randle (2009) and Prior et al. (1999). The EBSD technique as it is known today was first described in a series of papers in the beginning of 1990s (Wright and Adams 1992, Adams et al. 1993, Kunze et al. 1993). These papers describe the first system with a computercontrolled SEM, a low-light CCD camera, a video card for on-line diffraction pattern acquisition and dedicate software for automatic indexation. Over the intervening years many other advances have occurred, including higher speeds for data acquisition, refinement of the Kikuchi bands analyses, the combination of EBSD and in situ chemical analyses via EDS/WDS for phase indexation and the development of the dynamical theory for EBSD

This article is protected by copyright. All rights reserved. 
diffraction patterns. Here we report on two recent publications devoted to (1) cryo-EBSD study of ice samples deformed experimentally and naturally and (2) ultra-high resolution EBSD in FIB-prepared foils.

\section{Recent milestone EBSD applications in geochemistry}

Cryo-EBSD and crystallographic orientations in ice: Natural ice is a major component of the Earth's polar regions and it plays a fundamental role in our planet's climate system, affecting sea level and consequently the ocean currents that control temperatures, seasons and rainfall distribution on our planet. The effect of polar ice discharge into the oceans is nevertheless poorly understood, mainly because of an incomplete understanding of solid-state flow in ice sheets. In essence, ice flow involves either sliding over rocks at the base of the ice or internal flow, usually involving deformation and recrystallisation within the ice body. Such mechanisms operate essentially at the macroscopic scale, but a complete understanding of the physical processes involved in such deformation requires that the microstructures and mechanical properties of naturally and experimentally deformed ice be investigated. Furthermore, a better understanding of the rheological behaviour of ice may yield insights about the dynamics of giant gas planets and icy moons. Up until a few years ago the study of ice was very challenging, as it requires a cryogenic system coupled with the analytical or deformation tool, further requiring controls on temperature, pressure, water fugacity, etc. The first attempts to measure crystallographic orientations via EBSD on ice were made in the early 2000s (Iliescu et al. 2004), but it was not before the work of Weikusat et al. (2010) that the method became established. Only recently has the routine mapping of crystallographic orientations in ice via cryo-EBSD become available, lead by the group at the University of Otago, New Zealand (see also Prior et al. 2012).

Prior et al. (submitted) have describe the procedures they developed and successfully applied to the analyses of $\sim 100$ samples of synthetic and natural ice, addressing both a range of grain sizes and levels of impurities. EBSD mapping needs flat sample surfaces free of mechanical damage, as the electron backscatter diffraction signal arises $\leq 20 \mathrm{~nm}$ from the sample surface. Sample preparation for rocks normally evolves a mechanical and a chemical-mechanical polishing step, where some materials may require etching, electro- or ion-polishing carried out under carefully controlled conditions. It was therefore necessary that such methods should be adapted for ice sample preparation, where a very important factor is that the sample

This article is protected by copyright. All rights reserved. 
surface must be free of frost particles. During preparation of ice samples it is essential to track the thermal history of the sample, from synthesis and storage to cutting and polishing, as microstructural modifications are prone to occur even if only small temperature variations occur. After synthesis sample storage is a very important consideration; fine-grained samples (10-20 $\mu \mathrm{m}$ grain sizes) require storage in liquid nitrogen, whereas standard ice (grain sizes around $500 \mu \mathrm{m})$ can be stored in low temperature freezers $\left(-80^{\circ} \mathrm{C}\right)$. For sample cutting the authors used both a scroll saw designed to cut wood and a circular saw with a diamondsintered blade, as would be used in rock sample preparation. For obvious reasons, cutting (and polishing) has to be done in cold rooms, with temperatures varying from -10 to $-15{ }^{\circ} \mathrm{C}$. In addition, a series of precautions/procedures have to be considered so as to avoid any modifications to the microstructure of the samples (pre-chilled transfer cool boxes, precooled saw guide, etc.). Optimal cutting routines depend on the sample properties, where medium grained samples without mechanical anisotropies might be cut with a circular saw whereas more delicate, fine grained samples need to be cut with a scroll saw. Care during sample mounting is crucial if microstructures are to be preserved, hence Prior et al. (submitted) developed cooper sample holders to which the samples could be attached and stored, without the need to remount the samples in the case of reanalysis. Sample mounting is involved the melting and refreezing of a thin layer at the bottom of the sample to make the bond between the material being investigated and the holder. To minimise thermal impact, the very cold ice is pushed against the holder at $5-10{ }^{\circ} \mathrm{C}$ until a melt layer $<1 \mathrm{~mm}$ is created. Then, as soon as the bond is made, the sample holder was pressed against another sample holder at liquid nitrogen temperature. To obtain flat surfaces in their samples, particularly in coarse grained samples, the authors used a cryo-microtome that could be cooled to $-30{ }^{\circ} \mathrm{C}$. Flat surface samples are also obtained by grinding the samples on a metal plate cooled with liquid nitrogen, assuring that the sample surface remains below $100{ }^{\circ} \mathrm{C}$ during the first $20 \mathrm{~s}$ of grinding, which is enough time to generate a flat surface. For the sample transfer from the storage boxes to the SEM, the authors used a nitrogen glove box (Figure 6A) that could be fixed directly to the vacuum chamber door, allowing sample exchanging under a controlled atmosphere. This arrangement allowed the use of the main SEM vacuum chamber door, rather than through the commonly used airlock, thereby overcoming the problem of some cryo-transfer systems that limit sample tilt and movement, precluding EBSD measurements. The cryo-stage is made of $\mathrm{Cu}$ with a liquid nitrogen reservoir with an internal thermocouple and a heating element. The setup procedure for the ice EBSD took between 1 and $2 \mathrm{hr}$, depending on the experience of the users. Prior et al. (submitted) report that the last step

This article is protected by copyright. All rights reserved. 
before the EBSD mapping or imaging involves surface sublimation to remove frost and damage caused by cutting and grinding. Sublimation, employing the cryo-stage's internal heater operating under high-vacuum, was found to be effective for coarse-grained samples but not successful in fine-grained ice. These authors go on to describe two strategies for inducing sublimation.

$<<$ Figure 6 approximately here; colour $>>$

All analytical work was carried with a Zeiss Sigma VP FEG-SEM coupled with a NordlysF camera using AZTEC software from Oxford Instruments. Image acquisition and EBSD mapping were conducted in the variable pressure mode, usually with $\mathrm{N}$ as process gas. For the image acquisition Prior et al. (submitted) used different accelerating voltages and beam currents, whereas all EBSD measurements were acquired with a $30 \mathrm{kV}$ accelerating voltage and a $90 \mathrm{nA}$ beam current, albeit using variable working distances. An example from an ice orientation map is shown in Figure 6B, where the colour variations record changes in crystallographic orientation. These authors demonstrated that cryo-EBSD is a powerful technique for understanding ice microstructural behaviour and ice transformation mechanisms. In addition, this analytical approach makes possible the study of recrystallisation processes and the orientation between parent and recrystallised grains. Although this is also possible in normal EBSD analyses, unlike most natural minerals some crystallographic types of ice are free of twinning, allowing the isolated study of recrystallisation free of the twinning effects. Ice EBSD is also well suited for better constraining the microstructural evolution of experimentally and naturally deformed samples. Such work further constrains the kinetics and mechanical behaviour related to grain size reduction during ice deformation. One minor issue for this technique is the fact that the grain sizes in some natural samples (e.g., glacial ice) reach up to the $\mathrm{cm}$-scale, making truly representative EBSD mapping difficult. Nevertheless, the authors successfully measured the crystallographic orientations of samples larger than $7 \mathrm{~cm}$ in length, with very high resolution. This is the first time that EBSD mapping has been carried out at this scale. One can expect much more research to be reported on this topic in the coming few years.

Ultra-high resolution EBSD in FIB-prepared foils: Since the advent of EBSD techniques a couple decades ago, scientists have struggled to achieve near-nanometre resolution in crystallographic orientation determinations using scanning electron 
microscopes. Although constant improvements have been made on both field emission gun technologies (the best electron source so far) and EBSD detectors since the 1980s, the basic principles of electron-matter interaction in SEMs (volume interaction) and the high tilt angle associated with this technique have prevented resolutions below some tens of nanometres. For many years, material researchers, and more recently Earth scientists, have been interested in ultra-fine grained materials, as their physical properties (e.g., strength and plasticity) have very different behaviours to those observed at coarser grained scales. As an example, nanocrystalline particles are thought to have a major effect on the strength of upper crustal faults, and their presence or absence may influence the seismic vs. aseismic nature in these places. In addition, nanocrystals are commonly present in biominerals, they occur as inclusions in many different minerals and they can influence the early stages of crystallisation. Although standard EBSD has been commonly applied to the characterisation of fine-grained materials, its spatial resolution has not been sufficient for the routine characterisation of nanostructured materials, meaning most such studies have had to be based on transmission electron microscopy. In a recent paper Trimby (2012) proposed a solution for this problem, employing what the author has termed "transmission Kikuchi diffraction" (TKD); this seems to offer a much better spatial resolution than standard EBSD analyses. The key innovation in the TKD method is its use of transparent samples prepared for TEM analyses in conjunction with the sample being mounted at $90^{\circ}$ from the traditional EBSD position (Figure 6C). Under these conditions, good quality diffraction patterns could be obtained from domains below $10 \mathrm{~nm}$, allowing the detailed mapping of crystallographic orientations at such a fine scale. The author used two TEM-prepared foils of aluminium alloy and nanocrystalline nickel as illustrative test materials, from which he went on to measure successfully samples of diamond with nano-inclusions of iron oxides and sulfides (Figure 6D). These foils were mounted using a micro-clamp SEM sub-stage attached to a standard $70^{\circ}$ tilted sample holder. The SEM stage is then tilted $20^{\circ}$ towards the EBSD detector, so the TEM foils lie in a horizontal position (Figure 6C). The author used a very small working distance $(5 \mathrm{~mm})$, whereas the EBSD camera was located in the standard operating position. Trimby (2012) used accelerating voltages between 20-28 kV and beam currents between 1$10 \mathrm{nA}$ on the University of Sydney's Zeiss Ultra Plus FEG SEM equipped with a Nordlys-S EBSD detector and Channel 5 suite of programs, both from Oxford Instruments. As the sample lies in the horizontal, no tilt correction or dynamic focus correction was necessary. The EBSD patterns produced with the TKD-SEM technique was very sharp, and it was shown that the thickness of the sample plays an important role in determine the quality of the

This article is protected by copyright. All rights reserved. 
patterns. If the sample is too thick, two or more superimposed patterns may occur and the indexation becomes impossible, whereas if the sample is too thin the diffraction contrast is low and the patterns are again difficult to index. According to the author, good diffraction patterns can be obtained in samples ranging from 75 to $400 \mathrm{~nm}$ thick when using a $22 \mathrm{kV}$ accelerating voltage, however the optimal thickness values were found to depend on the accelerating voltage used during analysis. High resolution mapping of both samples (with an average grain size of $200 \mathrm{~nm}$ ) was achieved and, as can be seen in Figure 6D, the same resolution could also be obtained from minerals. The results from Trimby (2012) indicate a true spatial resolution of 5-10 $\mathrm{nm}$ for the nanocrystalline nickel sample, and around $10 \mathrm{~nm}$ for the $\mathrm{Al}$ alloy; it would appear that similar resolutions are possible for minerals in general. The work performed by the author suggests that this technique can be used to map grains well below $100 \mathrm{~nm}$ - this technique will certainly attract the attention of Earth scientists in the near future.

\section{Outlook for EBSD in the Geosciences}

With respect to EBSD investigations of ice, some issues remain regarding porous samples (e.g., snow) that are very delicate, and it appears further work will be needed on refining the sample preparation before these will be successfully measured. In addition, "dirty ice" (mixture of ice and rock particles) has also proven challenging to prepare because the hardness contrast between the clasts and ice. This means that standard preparation strategies may lead to topographic effects on the sample surface, inhibiting precise crystal orientation determinations. EBSD on different ice polymorphs is also something warranting further exploration, but this will require modifications being made on existing cryo-systems (e.g., lower temperatures). Other types of ices, such as $\mathrm{CO}_{2}$ and $\mathrm{CH}_{4}$, which are major components on giant gas planets, have yet to be explored; here there seems to be great potential for future studies.

The transmission Kikuchi diffraction in SEM is likely to attract a lot of interest in the near future due to its high spatial resolution and ease of data acquisition; importantly, such measurements can be made using a standard SEM with field emission gun and EBSD detector. The study performed by Trimby (2012) has clearly shown that the TKD can resolve structures that had previously proven difficult or impossible to resolve using standard EBSD methodologies. The effect of high intra-crystalline defect densities and the study of samples

This article is protected by copyright. All rights reserved. 
whose degree of crystallinity is so low that individual grains cannot be resolved by traditional EBSD are potential candidates to be studied with the TKD-SEM technique. In addition, this method may prove ideal for the study of FIB milled tips prior to atom probe analyses (e.g., Yao et al. 2011, see also atom probe chapter in this paper). As the sample during TKD investigation lies in a horizontal position, EDS mapping coupled with EBSD techniques can be used to separate phases with similar crystal structures at high spatial resolution.

\section{Atom probe tomography in the geosciences}

(Contribution by: M. Wiedenbeck)

\section{Overview of the atom probe method}

Although it has only recently been taken up in the geosciences, atom probe tomography (APT) has actually existed as an analytical tool since roughly 1970. This technology offers the unique and amazing capability of mapping the chemical and structural composition of a material at the atomic scale, generating massive data sets describing the position and isobaric identity of individual atoms within a 3-dimensional volume. Basically, an atom probe consists of a field emission ion source (the sample) coupled to a time-of-flight mass spectrometer, where ion detection uses a position sensitive detector capable of recording the arrival times of individual ions. The ion optics of such a system are designed to provide a magnification on the order of $10^{6}$, allowing the $\mathrm{X}-\mathrm{Y}$ position recorded by the ion detection system to be transposed to the original location of a particular atom at a sub-nanometre scale (Figure 7). As the system depends on a field emission ion source, the material under investigation must be prepared as a sharp tip with a diameter on the scale of up to a few 100's of nanometre in diameter and with a length typically around $1000 \mathrm{~nm}$, meaning sample preparation generally requires ion milling using focused ion beam technology. Another challenge of APT technology had earlier been the need to pulse the voltage applied to the sample in order to generate a start time-stamp for the time-of-flight system. This need to apply rapid voltage shifts to the sample tip largely precluded the analysis of non-conducting materials, as they tend to break under due to mechanical stress. However, with the advent of pulsed laser evaporation, the attractiveness of APT technology for investigating semiconductors and non-conductors has recently improved. APT systems have been commercially available for perhaps a decade, meaning the atomic-scale characterisation of non-conducting geomaterials is now realistic. A detailed description of APT technology is provided by Gault et al. (2012) and a brief overview of the historical background is provided by Wiedenbeck (2011).

This article is protected by copyright. All rights reserved. 
$<<$ Figure 7 approximately here; colour $>>$

\section{APT applications in mineralogy and geochemistry}

As laser assisted APT has existed for only a relatively short time, until now this technology has found few applications for Earth science topics; this is the first time that APT technology has been reported in this biennial review series. A fundamental investigation of the potential of the atom probe as applied to mineralogy was published by Bachhav et al. (2011), who report an APT study of wüstite $\left(\mathrm{Fe}_{1-\mathrm{x}} \mathrm{O}\right)$, which is of interest in both the material sciences as well as the Earth sciences. These authors assessed the significance of such parameters as laser energy, sample temperature and the voltage applied to the sample during analysis. Their paper documents that by varying the laser conditions used during analysis one alters significantly the quantitative results - in this particular study the goal having been the determination of the absolute oxygen content of the sample. Another valuable contribution by Bachhav et al. (2011) is their description of the sample preparation requirements of their instrument. Although somewhat on the brief side, this description gives good insight into the complexity of the atom probe method. Another interesting observation presented in this work is that a large spectrum of both multiply charged and molecular ions could be detected, despite the relatively simple chemistry of the wüstite material. This work lays the foundations for future fundamental studies needed in order to convert APT, which currently can be seen as an extremely high spatial resolution visualisation tool, into a truly quantitative analytical technique. Obvious points for further investigation are the role of the system's mass resolving power and the detection limits for minor components, which are largely defined by detector noise. One shortcoming acknowledged by Bachhav et al. (2011) was the inability of the

system to reliably detect the minor isotope ${ }^{18} \mathrm{O}$, which would otherwise have been useful for distinguishing between ${ }^{16} \mathrm{O}^{+}$and ${ }^{16} \mathrm{O}_{2}{ }^{2+}$ on the $\mathrm{m} / \mathrm{e}=16$ mass station; distinguishing between these two species was of fundamental importance in view of the goal of quantifying the absolute oxygen content of the material. Though not discussed by these authors, information about the presence or absence of a signal at the $\mathrm{m} / \mathrm{e}=17$ mass station would have been highly relevant, revealing the abundance of the ${ }^{16} \mathrm{O}^{18} \mathrm{O}^{2+}$ species. Future investigation into detector background, system mass resolution, ion through-put rates and factors affecting the detection of minor and trace components will be valuable contributions towards understanding the strengths and limitation of the atom probe method.

Until now only a single paper using APT for a purely Earth science application has appeared in the scientific literature. Valley et al. (2014) used laser assisted APT to image nanostructures within the core of a zircon that had previous been dated by SIMS to be nearly 4.4 Ga. This publication is not only noteworthy for its pioneering use of APT technology, but

This article is protected by copyright. All rights reserved. 
is also valuable for an appendix describing the analytical method in detail. These authors found a plethora of trace element enriched clusters (Figure 8) spread throughout the volume that they analysed. These enriched zones were defined by the authors as those volumes that their imaging software determined to have 3 at $\%$ or more of the trace element yttrium, which is one to two orders more enriched than that detected in the surrounding matrix. Many other trace elements were found to behave similarly. Of particular interest was that the ${ }^{207} \mathrm{~Pb} /{ }^{206} \mathrm{~Pb}$ ratio - measured using the ${ }^{206} \mathrm{~Pb}^{2+}$ and ${ }^{207} \mathrm{~Pb}^{2+}$ mass stations, as these were largely free of isobaric interferences - differed between the two classes of material. The ${ }^{207} \mathrm{~Pb} /{ }^{206} \mathrm{~Pb}$ ratio was found to be $1.25 \pm 0.05$ and $0.30 \pm 0.05(2 s)$ for the cores of yttrium-rich clusters and the non-enriched matrix, respectively. Valley et al. (2014) interpret their results from the yttriumrich volumes as recording radiogenic $\mathrm{Pb}$ produced between 4.4 and $3.4 \mathrm{Ga}$, whereas that in the case of the matrix material recording uranium decay over the past $3.4 \mathrm{Ga}$. The age of 3.4 Ga had been previously determined by SIMS on an overgrowth on this particular zircon and has been interpreted as the time of a high temperature event of regional significance. The cluster-like nature of the trace element enriched volumes is postulated to be the result of localised lattice damage resulting from $\alpha$-recoil cascades, which were annealed during the subsequent metamorphism.

$<<$ Figure 8 approximately here; colour $>>$

The work by Valley et al. (2014) may well prove to be a watershed publication, for the first time undertaking isotopic analyses at the near-atomic scale. This suggests great potential of the APT method as applied to geoscience research. Equally clear from this contribution is the huge amount of work needed prior to this method becoming a "routine" method. Many questions remain unaddressed, including the issues of detector backgrounds, magnitude and potential means for suppressing polyatomic interferences and the relative abundances of various charge states of the elements of interest. Also of potentially great significance is the topic of using the time-of-flight nature of APT in organic geochemistry - is any useful molecular information retained through the field emission process? The low ion production rate of $\sim 0.008$ ions per cycle reported in this study, albeit with the laser operating at 200 $\mathrm{kHz}$, suggests that progress in reducing total analysis time to well below a full day might be possible. Increasing the ion yield above the reported level of $\sim 37 \%$ would also be of obvious benefit. Finally, for the specific case of the ${ }^{207} \mathrm{~Pb} /{ }^{206} \mathrm{~Pb}=1.25 \pm 0.05(2 s)$ determined for the yttrium-rich clusters reported by Valley et al. (2014), the value for radiogenic ${ }^{207} \mathrm{~Pb} /{ }^{206} \mathrm{~Pb}$ that evolved from 4.4 to $3.4 \mathrm{Ga}$ would be closer to ${ }^{207} \mathrm{~Pb} /{ }^{206} \mathrm{~Pb}=1.218$. Though the experimental result is within its assigned $2 s$ uncertainty of this value, this might suggest that other systematic processes such as unresolved isobars or detector background may warrant additional attention.

This article is protected by copyright. All rights reserved. 


\title{
Outlook for atom probe applications in mineralogy
}

Clearly the APT method has the potential to become a key method for the characterisation of geomaterials at the nanometre scale. As this technology has only recently become suitable for the analysis of electrically non-conducting specimens, many parameters remain to be investigated in order to optimise this approach for geochemical applications. The investigation of mineral grain boundaries, short-range diffusion and the characterisation of nano-inclusions - all of which require nm-scale lateral resolution - can be expected to benefit from atom probe technology. The ability to visualise structures in 3-dimensions at nanometre scales will also likely provide new perspectives on diffusion and reprecipitation mechanisms. One drawback to be highlighted about atom probe technology, however, is the need for extensive supporting infrastructure. Such instruments are not prohibitively expensive in themselves, but one needs to keep in mind that at a very minimum a scanning electron microscope plus focused ion beam capability is essential for their operation. Hence, it can be expected that APT will in the short to medium term be largely limited to either multi-user facilities or to exceptionally well-equipped laboratories with a focus on nano-scale processes. Nonetheless, as this class of instrumentation becomes more widely available, one can reasonably expect many new breakthrough discoveries from APT within the geosciences.

\section{Acknowledgements}

R. Bugoi and S. Merchel would like to thank discussions with C. Andermann, D.L. Bourlès, R. Braucher, M. Christl, F. Munnik and P. Steier.

\section{References}

Adams B.L., Wright S.I. and Kunze K. (1993)

Orientation imaging: The emergence of a new microscopy. Metallurgical Transactions, 24A, 819-831.

\author{
Akçar N., Deline P., Ivy-Ochs S., Alfimov V., Hajdas I., Kubik P.W., Christl M. and \\ Schlüchter C. (2012) \\ The AD 1717 rock avalanche deposits in the upper Ferret Valley (Italy): A dating approach \\ with cosmogenic ${ }^{10}$ Be. Journal of Quaternary Science, 27, 383-392.
}

This article is protected by copyright. All rights reserved. 
Akçar N., Ivy-Ochs S., Deline P., Alfimov V., Kubik P.W., Christl M. and Schlüchter C. (2014)

Minor inheritance inhibits the calibration of the ${ }^{10} \mathrm{Be}$ production rate from the AD $1717 \mathrm{Val}$

Ferret rock avalanche, European Alps. Journal of Quaternary Science, 29, 318-328.

Amarasiriwardena D., Krushevska A., Argentine M. and Barnes R.M. (1994)

Vapor-phase acid digestion of micro samples of biological-material in a high-temperature, high pressure asher for inductively coupled plasma-atomic emission spectrometry. The Analyst, 119, 1017-1021.

Andersen C.A. and Hinthorne J.R. (1972)

$\mathrm{U}, \mathrm{Th}, \mathrm{Pb}$ and REE abundances and ${ }^{207} \mathrm{~Pb} /{ }^{206} \mathrm{~Pb}$ ages of individual minerals in returned Lunar material by ion microprobe mass analysis. Earth and Planetary Science Letters, 14, 195200 .

Bachhav M., Danoix R., Danoix F., Hannoyer B., Ogale S. and Vurpillot F. (2011) Investigation of wüstite $\left(\mathrm{Fe}_{1-\mathrm{x}} \mathrm{O}\right)$ by femtosecond laser assisted atom probe tomography. Ultramicroscopy, 111, 584-588.

Balaram V., Vummiti D., Roy P., Taylor C., Kamala C.T., Satyanarayanan M., Kar P., Subramanyam K.S.V., Raju A.K. and Abburi K. (2014)

Analysis of geochemical samples by microwave plasma-AES. Atomic Spectroscopy, 35, $65-78$.

Balaram V., Vummiti D., Roy P., Taylor C., Kar P., Raju A.K. and Abburi K. (2013) Determination of precious metals in rocks and ores by microwave plasma-atomic emission spectrometry for geochemical prospecting studies. Current Science, 104, 1207-1215.

Bamba S., Yamaguchi K.E. and Amano H. (2014)

Determination of ${ }^{129} \mathrm{I} /{ }^{127} \mathrm{I}$ in environmental water before and after the 2011 Fukushima

Daiichi nuclear power plant accident with a solid extraction disk. Journal of

Radioanalytical and Nuclear Chemistry, 301, 75-80.

Becker J.S., Zoriy M., Matusch A., Wu B., Salber D. and Palm C. (2010)

Bioimaging of metals by laser ablation inductively coupled plasma-mass spectrometry (LAICP-MS). Mass Spectrometry Reviews, 29, 156-175.

Bédard L.P. and Néron A. (2014)

This article is protected by copyright. All rights reserved. 
Spatial geochemistry: A characterisation of heterogeneity in reference materials.

Geostandards and Geoanalytical Research, 38, 177-188.

Bierman P.R., Corbett L.B., Graly J.A., Neumann T.A., Lini A., Crosby B.T. and Rood D.H. (2014)

Preservation of a preglacial landscape under the center of the Greenland ice sheet. Science, 344, 402-405.

Borysiuk M., Ros L., Kristiansson P., Skogby H., Abdel N., Elfman M., Golubev P., Nilsson E.J.C. and Pallon J. (2013)

Hydrogen analysis and profiling with a position sensitive detector. Nuclear Instruments and Methods in Physics Research B, 306, 49-53.

Borysiuk M., Kristiansson P., Ros L., Abdel N., Elfman M., Nilsson E.J.C. and Pallon J. (2014)

Evaluation of a setup for pNRA at LIBAF for applications in geosciences. Nuclear

Instruments and Methods in Physics Research B, 332, 202-206.

Bowring J., Horstwood M. and Gehrels G. (2013)

Resolving bias in laser ablation geochronology: Workshop on data handling in LA-ICPMS U-Th-Pb geochronology, Charleston, South Carolina, 6-8 March 2013. EOS, Transactions American Geophysical Union, 94 (24), 217.

Briner J.P., Young N.E., Goehring B.M. and Schaefer J.M. (2012)

Constraining Holocene ${ }^{10} \mathrm{Be}$ production rates in Greenland. Journal of Quaternary Science, 27, 2-6.

Calligaro T., Coquinot Y., Pichon L, Pierrat-Bonnefois G., de Campos P., Re A. and Angelici D. (2014)

Characterization of the lapis lazuli from the Egyptian treasure of Tôd and its alteration using external $\mu$-PIXE and $\mu$-IBIL. Nuclear Instruments and Methods in Physics Research B, 318, 139-144.

Casacuberta N., Christl M., Lachner J., Rutgers van der Loeff M., Masqué P. and Synal H.-A. (2014)

A first transect of ${ }^{236} \mathrm{U}$ in the North Atlantic Ocean. Geochimica et Cosmochimica Acta, 133, 34-46. 
Chaves P.C., Taborda A., de Oliveira D.P.S. and Reis M.A. (2014a)

CdTe detector based PIXE mapping of geological samples. Nuclear Instruments and Methods in Physics Research B, 318, 37-41.

Chaves P.C., Taborda A., Marques J.P. and Reis M.A. (2014b)

$\mathrm{N}$ to K Uranium PIXE spectra obtained at the high resolution high energy PIXE setup.

Nuclear Instruments and Methods in Physics Research B, 318, 60-64.

Chew D.M., Petrus J.A. and Kamber B.S. (2014)

$\mathrm{U}-\mathrm{Pb}$ LA-ICPMS dating using accessory mineral standards with variable common $\mathrm{Pb}$.

Chemical Geology, 363, 185-199.

Christl M., Lachner J., Vockenhuber C., Goroncy I., Herrmann J. and Synal H.-A. (2013)

First data of uranium-236 in the North Sea. Nuclear Instruments and Methods in Physics

Research B, 294, 530-536.

Christl M., Dai X., Lachner J., Kramer-Tremblay S. and Synal H.-A. (2014)

Low energy AMS of americium and curium. Nuclear Instruments and Methods in Physics

Research B 331, 225-232.

Cnudde V. and Boone M.N. (2013)

High-resolution X-ray computed tomography in geosciences: A review of the current technology and applications. Earth-Science Reviews, 123, 1-17.

Collon P., Bowers M., Calaprice F., Galbiati C., Henderson D., Hohman T., Jiang C.L., Kutschera W., Lee H.Y., Loer B., Pardo R.C., Paul M., Rehm E., Robertson D., Schmitt C., Scott R. and Vondrasek R. (2012)

Reducing potassium contamination for AMS detection of ${ }^{39} \mathrm{Ar}$ with an electron-cyclotronresonance ion source. Nuclear Instruments and Methods in Physics Research B, 283, 77 83.

Cotta A.J.B. and Enzweiler J. (2012)

Classical and new procedures of whole rock dissolution for trace element determination by ICP-MS. Geostandards and Geoanalytical Research, 36, 27-50.

Cotta A.J.B., Enzweiler J., Wilson S.A., Perez C.A., Nardy A.J.R. and Larizzatti J.H. (2007) 
Homogeneity of the geochemical reference material BRP-1 (Parana Basin Basalt) and assessment of minimum mass. Geostandards and Geoanalytical Research, 31, 379-393.

Cottle J.M., Horstwood M.S.A. and Parrish R.R. (2009)

A new approach to single shot laser ablation analysis and its application to in situ $\mathrm{Pb} / \mathrm{U}$ geochronology. Journal of Analytical Atomic Spectrometry, 24, 1355-1363.

Cottle J.M., Kylander-Clark A.R. and Vrijmoed J.C. (2012)

$\mathrm{U}-\mathrm{Th} / \mathrm{Pb}$ geochronology of detrital zircon and monazite by single shot laser ablation inductively coupled plasma-mass spectrometry (SS-LA-ICP-MS). Chemical Geology, 332, $136-147$.

Cottle J.M., Burrows A.J., Kylander-Clark A., Freedman P.A. and Cohen R.S. (2013)

Enhanced sensitivity in laser ablation multi-collector inductively coupled plasma-mass spectrometry. Journal of Analytical Atomic Spectrometry, 28, 1700-1706.

Crowe D.E. and Vaughan R.G. (1996)

Characterization and use of isotopically homogeneous standards for in situ laser microprobe analysis of ${ }^{34} \mathrm{~S} /{ }^{32} \mathrm{~S}$ ratios. American Mineralogist, 81, 187-193.

Dehlinger M., Fauquet C., Jandard F., Bjeoumikhov A., Bjeoumikhova S., Gubzhokov R., Erko A., Zizak I., Pailharey D., Ferrero S. and Tonneau D. (2013) Toward sub-micro-XRF working at nanometer range using capillary optics. X-Ray Spectrometry, 42, 456-461.

Delunel R., Bourlès D.L., van der Beek P.A., Schlunegger F., Leya I., Masarik J. and Paquet E. (2014)

Snow shielding factors for cosmogenic nuclide dating inferred from long-term neutron detector monitoring. Quaternary Geochronology, 24, 16-26.

Devès G., Perroux A.S., Bacquart T., Plaisir C., Rose J., Jaillet S., Ghaleb B., Ortega R. and Maire R. (2012)

Chemical element imaging for speleothem geochemistry: Application to a uranium-bearing corallite with aragonite diagenesis to opal (Eastern Siberia, Russia). Chemical Geology, 294, 190-202.

Donati G.L., Amais R.S., Schiavo D. and Nobrega J.A. (2013)

This article is protected by copyright. All rights reserved. 
Determination of $\mathrm{Cr}, \mathrm{Ni}, \mathrm{Pb}$ and $\mathrm{V}$ in gasoline and ethanol fuel by microwave plasma optical emission spectrometry. Journal of Analytical Atomic Spectrometry, 28, 755-759.

Dunai T.J., Binnie S.A., Hein A.S. and Paling S.M. (2014)

The effects of a hydrogen-rich ground cover on cosmogenic thermal neutrons: Implications for exposure dating. Quaternary Geochronology, 22, 183-191.

Engler O. and Randle V. (2009)

Introduction to texture analysis macrotexture, microtexture, and orientation mapping. CRC Press, Taylor and Francis Group (Boca Raton, USA).

Fan Y., Hou X. and Zhou W. (2013)

Progress on ${ }^{129} \mathrm{I}$ analysis and its application in environmental and geological researches. Desalination, 321, 32-46.

Fayek M. (2009)

Secondary ion mass spectrometry in the Earth sciences. Mineralogical Association of Canada, Short Course Series, 41, 148pp.

Finkel R., Arnold M., Aumaître G., Benedetti L., Bourlès D., Keddadouche K. and Merchel S. (2013)

Improved ${ }^{36} \mathrm{Cl}$ performance at the ASTER HVE $5 \mathrm{MV}$ accelerator mass spectrometer national facility. Nuclear Instruments and Methods in Physics Research B, 294, 121-125.

Garapic G., Faul U.H. and Brisson E. (2013)

High-resolution imaging of the melt distribution in partially molten upper mantle rocks: Evidence for wetted two-grain boundaries. Geochemistry Geophysics Geosystems, 14, doi:10.1029/2012GC004547.

Gault B., Moody M.P., Cairney J.M. and Ringer S.P. (2012)

Atom probe microscopy. Springer Series in Material Sciences. Springer Verlag, 396pp.

Gerdes A. and Zeh A. (2009)

Zircon formation versus zircon alteration - New insights from combined U-Pb and Lu-Hf insitu LA-ICP-MS analyses, and consequences for the interpretation of Archaean zircon from the Central Zone of the Limpopo Belt. Chemical Geology, 261, 230-243.

This article is protected by copyright. All rights reserved. 
Gibbon R.J., Pickering T.R., Sutton M.B., Heaton J.L., Kuman K., Clarke R.J., Brain C.K. and Granger D.E. (2014)

Cosmogenic nuclide burial dating of hominin-bearing Pleistocene cave deposits at Swartkrans, South Africa. Quaternary Geochronology, 24, 10-15.

Granger D.E., Lifton N.A. and Willenbring J.K (2013a)

A cosmic trip: 25 years of cosmogenic nuclides in geology. Bulletin of the Geological Society of America, 125, 1379-1402.

Granger D.E., Rogers H.E., Riebe C.S. and Lifton N.A (2013b)

Production rate of cosmogenic ${ }^{10} \mathrm{Be}$ in magnetite. American Geophysical Union, Fall Meeting 2013, abstract \#EP53A-0736.

Guillong M. and Heinrich C.A. (2007)

Sensitivity enhancement in laser ablation ICP-MS using small amounts of hydrogen in the carrier gas. Journal of Analytical Atomic Spectrometry, 22, 1488-1494.

Hammer M.R. (2008)

A magnetically excited microwave plasma source for atomic emission spectroscopy with performance approaching that of the inductively coupled plasma. Spectrochimica Acta Part B, 63, 456-464.

Hartley M.E., Thordarson T., Taylor C., Fitton J.G. and EIMF (2012)

Evaluation of the effects of composition on the instrumental mass fractionation during SIMS oxygen isotope analyses of glasses. Chemical Geology, 334, 312-323.

Hinton R. (1995)

Ion microprobe analysis in geology. In: Potts P.J., Bowles J.F.W., Reed S.J.B and Cave M.R. (eds), Microprobe techniques in the Earth sciences. Chapman and Hall, 235-289.

Holt M., Harder R., Winarski R. and Rose V. (2013)

Nanoscale hard X-ray microscopy methods for materials studies. Annual Review of Materials Research, 43, 183-211.

Hoppe P., Cohen S. and Meibom A (2013)

NanoSIMS: Technical aspects and applications in cosmochemistry and biological geochemistry. Geostandards and Geoanalytical Research, 37, 111-154.

This article is protected by copyright. All rights reserved. 
Horstwood M.S.A. (2008)

Data reduction strategies, uncertainty assessment and resolution of LA-(MC-)ICP-MS isotope data. In: Sylvester P. (ed.), Laser ablation ICP-MS in the Earth sciences: Current practices and outstanding issues. Mineralogical Association of Canada, Short Course Series, 40, 283-300.

Horstwood M., Gehrels G. and Bowring J. (2010)

Improving consistency in laser ablation geochronology: Workshop on data handling in LAICP-MS U-Th-Pb geochronology, San Francisco, California, 12-13 December 2009. EOS, Transactions American Geophysical Union, 91 (28), 247.

Hu Z.C., Gao S., Liu Y.S., Hu S.H., Chen H.H. and Yuan H.L. (2008)

Signal enhancement in laser ablation ICP-MS by addition of nitrogen in the central channel gas. Journal of Analytical Atomic Spectrometry, 23, 1093-1101.

Hu Z., Liu Y., Gao S., Liu W., Zhang W., Tong X., Lin L., Zong K., Li M., Chen H., Zhou L. and Yang L. (2012)

Improved in situ $\mathrm{Hf}$ isotope ratio analysis of zircon using newly designed $\mathrm{X}$ skimmer cone and jet sample cone in combination with the addition of nitrogen by laser ablation multiple collector ICP-MS. Journal of Analytical Atomic Spectrometry, 27, 1391-1399.

Hu Z., Zhang W., Liu Y., Chen H., Gaschnig R.M., Zong K., Li M., Gao S. and Hu S. (2013)

Rapid bulk rock decomposition by ammonium fluoride $\left(\mathrm{NH}_{4} \mathrm{~F}\right)$ in open vessels at an elevated digestion temperature. Chemical Geology, 355, 144-152.

Huber C., Smolek S. and Streli C. (2014)

Simulation of layer measurement with confocal micro-XRF. X-Ray Spectrometry, 43, 175179 .

Ichikawa S. and Nakamura T. (2014)

$\mathrm{X}$-ray fluorescence analysis with micro glass beads using milligram-scale siliceous samples for archaeology and geochemistry. Spectrochimica Acta Part B, 96, 40-50.

Ickert R.B. and Stern R.A. (2013)

Matrix corrections and error analysis in high-precision SIMS ${ }^{18} \mathrm{O} /{ }^{16} \mathrm{O}$ Measurements of $\mathrm{Ca}-$ Mg-Fe Garnet. Geostandards and Geoanalytical Research, 37, 429-448.

This article is protected by copyright. All rights reserved. 
Iizuka T. and Hirata T. (2005)

Improvements in precision and accuracy in in situ $\mathrm{Hf}$ isotope microanalysis of zircon using the laser ablation-MC-ICPMS technique. Chemical Geology, 220, 121-137.

Iliescu D., Baker I. and Chang H. (2004)

Determining the orientations of ice crystals using electron backscatter patterns. Microscopy Research Technology, 63, 183-187.

Jankowski K. and Reszke E. (2013)

Recent developments in instrumentation of microwave plasma sources for optical emission and mass spectrometry: Tutorial review. Journal of Analytical Atomic Spectrometry, 28, 1196-1212.

Jansen J.D., Codilean A.T., Stroeven A.P., Fabel D., Hättestrand C., Kleman J., Harbor J.M., Heyman J., Kubik P.W. and Xu S. (2014)

Inner gorges cut by subglacial meltwater during Fennoscandian ice sheet decay. Nature Communications, 5, 3815.

Jean-Soro L., Oleron-Hamdous A., Béchet B. and Legret M. (2013)

Evaluation of platinum distribution between different soil components. Journal of Soils and Sediments, 13, 569-574.

Johnson J.S., Bentley M.J., Smith J.A., Finkel R.C., Rood D.H., Gohl K., Balco G., Larter R.D. and Schaefer J.M. (2014)

Rapid thinning of Pine Island glacier in the early Holocene. Science, 343, 999-1001.

Johnston S., Gehrels G., Valencia V. and Ruiz J. (2009)

Small-volume U-Pb zircon geochronology by laser ablation-multicollector-ICP-MS.

Chemical Geology, 259, 218-229.

Johnstone S., Hourigan J. and Gallagher C. (2013)

LA-ICP-MS depth profile analysis of apatite: Protocol and implications for (U-Th)/He thermochronometry. Geochimica et Cosmochimica Acta, 109, 143-161.

Jomelli V., Favier V., Vuille M., Braucher R., Martin L., Blard P.-H., Colose C., Brunstein D., He F., Khodri M., Bourlès D.L., Leanni L., Rinterknecht V., Grancher D., Francou B., Ceballos J.L., Fonseca H., Liu Z. and Otto-Bliesner B.L. (2014)

This article is protected by copyright. All rights reserved. 
A major advance of tropical Andean glaciers during the Antarctic cold reversal. Nature, 513, $225-228$.

Kanngießer B., Malzer W., Mantouvalou I., Sokaras D. and Karydas A.G. (2012)

A deep view in cultural heritage - Confocal micro X-ray spectroscopy for depth resolved elemental analysis. Applied Physics A, 106, 325-338.

Keller L.M., Schütz P., Erni R., Rossell M.D., Lucas F., Gasser P. and Holzer L. (2013) Characterization of multi-scale microstructural features in Opalinus Clay.

Microporous and Mesoporous Materials, 170, 83-94.

Kinoshita N., Paul M., Kashiv Y., Collon P., Deibel C.M., DiGiovine B., Greene J.P., Henderson D.J., Jiang C.L., Marley S.T., Nakanishi T., Pardo R.C., Rehm K.E., Robertson D., Scott R., Schmitt C., Tang X.D., Vondrasek R. and Yokoyama A. (2012) A shorter ${ }^{146} \mathrm{Sm}$ half-life measured and implications for ${ }^{146} \mathrm{Sm}-{ }^{142} \mathrm{Nd}$ chronology in the Solar System. Science, 335, 1614-1617.

Klein M., Heinemeier J., Gottdang A., Mous D.J.W. and Olsen J. (2014)

Extension of the HVE 1 MV multi-element AMS system for low background. Nuclear Instruments and Methods in Physics Research B, 331 204-208.

Koornneef J.M., Bouman C., Schwieters J.B. and Davies G.R. (2013)

Use of $10^{12} \mathrm{ohm}$ current amplifiers in $\mathrm{Sr}$ and $\mathrm{Nd}$ isotope analyses by TIMS for application to sub-nanogram samples. Journal of Analytical and Atomic Spectrometry, 28, 749-754.

Košler J. and Sylvester P.J. (2003)

Present trends and the future of zircon in geochronology: Laser ablation ICP-MS. In:

Hanchar J.M. and Hoskin P.W.O. (eds), Zircon. Reviews in Mineralogy and Geochemistry, 53, 243-275.

Košler J. (2008)

Laser ablation sampling strategies for concentration and isotope ratio analyses by ICP-MS. In: Sylvester P. (ed.), Laser ablation ICP-MS in the Earth sciences: Current practices and outstanding issues. Mineralogical Association of Canada, Short Course Series, 40, 79-92.

Košler J. (2012)

This article is protected by copyright. All rights reserved. 
$\mathrm{U}-\mathrm{Pb}$ geochronology and Hf-isotope geochemistry of detrital zircon in sedimentary systems. In: Sylvester P. (ed.), Quantitative mineralogy and microanalysis of sediments and sedimentary rocks. Mineralogical Association of Canada, Short Course Series, 42, 185202.

Košler J., Sláma J., Belousova E., Corfu F., Gehrels G.E., Gerdes A., Horstwood M.S.A., Sircombe K.N., Sylvester P.J., Tiepolo M., Whitehouse M.J. and Woodhead, J.D. (2013)

U-Pb detrital zircon analysis - Results of an inter-laboratory comparison. Geostandards and Geoanalytical Research, 37, 243-259.

Kristiansson P., Borysiuk M., Hålenius U., Mosenfelder J.L., Ros L., Skogby H., Abdel N., Elfman M., Nilsson E.J.C. and Pallon J. (2014)

The implementation of a DSSSD in the upgraded boron analysis at LIBAF for applications in geochemistry. Nuclear Instruments and Methods in Physics Research B, 332, 207-211.

Kristiansson P. and Martinsson B.G. (1997)

Photon-tagged nuclear reaction analysis for trace element determination. Nuclear Instruments and Methods in Physics Research B, 132, 159-176.

Kunze, K., Wright S.I., Adams B.L. and Dingley D.J. (1993)

Advances in automatic EBSP single orientation measurements. Textures and Microstuctures, 13, 41-45.

Kutschera W. (2013a)

Applications of accelerator mass spectrometry. International Journal of Mass Spectrometry 349-350, 203-218.

Kutschera W. (2013b)

Accelerator mass spectrometry - from DNA to astrophysics. EPJ Web of Conferences 63, 03001 .

Kylander-Clark A.R., Hacker B.R. and Cottle J.M. (2013) Laser-ablation split-stream ICP petrochronology. Chemical Geology, 345, 99-112.

Lachner J., Christl M., Alfimov V., Hajdas I., Kubik P.W., Schulze-König T., Wacker L. and Synal H.-A. (2014) 
${ }^{41} \mathrm{Ca},{ }^{14} \mathrm{C}$ and ${ }^{10} \mathrm{Be}$ concentrations in coral sand from the Bikini atoll. Journal of Environmental Radioactivity, 129, 68-72.

Laird J.S., Szymanski R., Ryan C.G. and Gonzalez-Alvarez I. (2013)

A Labview based FPGA data acquisition with integrated stage and beam transport control. Nuclear Instruments and Methods in Physics Research B, 306, 71-75.

Lal D. (1991)

Cosmic ray labelling of erosion surfaces: In situ nuclide production rates and erosion models. Earth and Planetary Science Letters, 104, 424-439.

Larsen I.J., Almond P.C., Eger A., Stone J.O., Montgomery D.R. and Malcolm B. (2014) Rapid soil production and weathering in the Southern Alps, New Zealand. Science, 343, 637640.

Leach A.M. and Hieftje G.M. (2000)

Methods for shot-to-shot normalization in laser ablation with an inductively coupled plasma time-of-flight mass spectrometer. Journal of Analytical Atomic Spectrometry, 15, 11211124.

Lebatard A.-E., Alçiçek M.C., Rochette P., Khatib S., Vialet A., Boulbes N., Bourlès D.L., Demory F., Guipert G., Mayda S., Titov V.V., Vidal L. and de Lumley H. (2014) Dating the Homo erectus bearing travertine from Kocabaş (Denizli, Turkey) at least 1.1 Ma. Earth and Planetary Science Letters, 390, 8-18.

Le Vaillant M., Barnes S.J., Fisher L., Fiorentini M.L. and Caruso S. (2014)

Use and calibration of portable X-Ray fluorescence analysers: Application to lithogeochemical exploration for komatiite-hosted nickel sulphide deposits. Geochemistry: Exploration, Environment, Analysis, 14, 199-209.

Lifton N., Sato T. and Dunai, T. (2014)

Scaling in situ cosmogenic nuclide production rates using analytical approximations to atmospheric cosmic-ray fluxes. Earth and Planetary Science Letters, 386, 149-160.

Linge K.L. and Jarvis K.E. (2009)

Quadrupole ICP-MS: Introduction to instrumentation, measurement techniques and analytical capabilities. Geostandards and Geoanalytical Research, 33, 445-467.

This article is protected by copyright. All rights reserved. 
Liu H., Mao X. and Russo R.E. (2001)

Representative sampling using single-pulse laser ablation with inductively coupled plasma mass spectrometry. Journal of Analytical Atomic Spectrometry, 16, 1115-1120.

Liu J. and Pearson D.G. (2014)

Rapid, precise and accurate Os isotope ratio measurements of nanogram to sub-nanogram amounts using multiple Faraday collectors and amplifiers equipped with $10^{12} \Omega$ resistors by N-TIMS. Chemical Geology, 363, 301-311.

Liu Y., Li X.-H., Li Q.-L., Tang G.-Q. And Yin Q.Z. (2011)

Precise U-Pb zircon dating at a scale of $<5$ micron by the CAMECA 1280 SIMS using a Gaussian illumination probe. Journal of Analytical and Atomic Spectrometry, 26, 845851.

Ludwig K.R. (2009)

Isoplot 4.1. A geochronological toolkit for Microsoft Excel. Berkeley Geochronology Center Special Publication (Berkeley), 4, 76pp.

\section{Lund C., Lamberg P. and Lindberg T. (2013)}

Practical way to quantify minerals from chemical assays at Malmberget iron ore operationsAn important tool for the geometallurgical program. Minerals Engineering, 49, 7-16.

Mason P.R.D. and Mank A.J.G. (2001)

Depth-resolved analysis in multi-layered glass and metal materials using laser ablation inductively coupled plasma mass spectrometry (LA-ICP-MS). Journal of Analytical Atomic Spectrometry, 16, 1381-1388.

McIntosh K.G., Neal J.A., Nath P. and Havrilla G.J. (2014)

Microfluidic sample preparation for elemental analysis in liquid samples using micro X-ray fluorescence spectrometry. X-Ray Spectrometry. doi: 10.1002/xrs.2559.

Meisel T., Moser J., Fellner N., Wegscheider W. and Schoenberg R. (2001)

Simplified method for the determination of $\mathrm{Ru}, \mathrm{Pd}, \mathrm{Re}, \mathrm{Os}$, Ir and Pt in chromitites and other geological materials by isotope dilution ICP-MS and acid digestion. The Analyst, 126, 322328.

Merchel S., Braucher R., Alfimov V., Bichler M., Bourlès D.L. and Reitner J.M. (2013)

This article is protected by copyright. All rights reserved. 
The potential of historic rock avalanches and man-made structures as chlorine-36 production rate calibration sites. Quaternary Geochronology 18, 54-62.

Merchel S., Mrak I., Braucher R., Benedetti L., Repe B., Bourlès D.L. and Reitner J.M. (2014)

Surface exposure dating of the Veliki vrh rock avalanche in Slovenia associated with the 1348 earthquake. Quaternary Geochronology 22, 33-42.

Miot J., Benzerara K. and Kappler A. (2014)

Investigating microbe-mineral interactions: Recent advances in X-ray and electron microscopy and redox-sensitive methods. Annual Review of Earth and Planetary Sciences, 42, 271-289.

Miyake Y., Matsuzaki H., Fujiwara T., Saito T., Yamagata T., Honda M. and Muramatsu Y. (2012)

Isotopic ratio of radioactive iodine $\left({ }^{129} \mathrm{I} /{ }^{131} \mathrm{I}\right)$ released from Fukushima Daiichi NPP accident. Geochemical Journal, 46, 327-333.

Montaser A., Ohls K.D. and Golightly D.W. (1992)

Inductively coupled plasmas in gases other than argon. In: Montaser A. and Golightly D.W. (eds), Inductively coupled plasmas in analytical atomic spectrometry. VCH Publishers (New York, USA), 877-948.

Mostafa M., El-gharbawy A.F., El-Absy M.A., Soliman S.E. and Aly F.A. (2014)

Preconcentration of radioiodine as AgI and purification from radiotellurium waste. Journal of Radioanalytical and Nuclear Chemistry, 300, 1089-1097.

Münsterer C., Wacker L., Hattendorf B., Christl M., Koch J., Dietiker R., Synal H.-A. and Günther D. (2014)

Rapid revelation of radiocarbon records with laser ablation accelerator mass spectrometry. CHIMIA International Journal for Chemistry, 68, 215-216.

Nakazawa T. and Tsuji K. (2013)

Depth-selective elemental imaging of microSD card by confocal micro XRF analysis. X-Ray Spectrometry, 42, 123-127.

This article is protected by copyright. All rights reserved. 
Nasdala L., Hofmeister W., Norberg N., Martinson J.M., Corfu F., Dörr W., Kamo S.L., Kennedy A.K., Kronz A., Reiners P.W., Frei D., Košler J., Wan Y, Götze J., Häger T., Kröner A. and Valley J.W. (2008)

Zircon M257 - A homogeneous natural reference material for the ion microprobe $\mathrm{U}-\mathrm{Pb}$ analysis of zircon. Geostandards and Geoanalytical Research, 32, 247-265.

Naumenko M.O., Mezger K., Nägler T.F. and Villa I.M. (2013)

High precision determination of the terrestrial ${ }^{40} \mathrm{~K}$ abundance. Geochimica et Cosmochimica Acta, 122, 353-362.

Nesbitt R.W., Hirata T., Bulter I.B. and Milton J.A. (1997)

UV laser ablation ICP-MS: some applications in the Earth Sciences. Geostandards

Newsletter: The Journal of Geostandards and Geoanalysis, 20, 231-243.

Newman K. (2012)

Effects of the sample interface in MC-ICP-MS: Relative elemental sensitivities and nonlinear mass dependent fractionation of $\mathrm{Nd}$ isotopes. Journal of Analytical Atomic Spectrometry, 28, 63-70.

Newman K., Freedman P.A., Williams J., Belshawb N.S. and Halliday A.N. (2009) High sensitivity skimmers and non-linear mass dependent fractionation in ICP-MS. Journal of Analytical Atomic Spectrometry, 24, 742-751.

Obst M. and Schmid G. (2014)

3D chemical mapping: Application of scanning transmission (soft) X-ray microscopy (STXM) in combination with angle-scan tomography in bio-, geo-, and environmental sciences. In: Electron Microscopy. (Humana Press), 757-781.

Osterholt T., Salber D., Matusch A., Becker J. and Palm C. (2011)

IMAGENA: Image generation and analysis - An interactive software tool handling LA-ICPMS data. International Journal of Mass Spectrometry, 307, 232-239.

Ott U., Besmehn A., Farouqi K., Hallmann O., Hoppe P., Kratz K.-L., Melber K. and Wallner A. (2012)

New attempts to understand nanodiamond stardust. Publications of the Astronomical Society of Australia, 29, 90-97.

Paton C., Hellstrom J., Paul B., Woodhead J. and Hergt J. (2011)

This article is protected by copyright. All rights reserved. 
Iolite: Freeware for the visualisation and processing of mass spectrometric data. Journal of Analytical Atomic Spectrometry, 26, 2508-2518.

Paul B., Paton C., Norris A., Woodhead J., Hellstrom J., Hergt J. and Greig A. (2012) CellSpace: A module for creating spatially registered laser ablation images within the Iolite freeware environment. Journal of Analytical Atomic Spectrometry, 27, 700-706.

Pavetich S., Akhmadaliev S., Arnold M., Aumaître G., Bourlès D., Buchriegler J., Golser R., Keddadouche K., Martschini M., Merchel S., Rugel G. and Steier P. (2014) Interlaboratory study of the ion source memory effect in ${ }^{36} \mathrm{Cl}$ accelerator mass spectrometry. Nuclear Instruments and Methods in Physics Research B, 329, 22-29.

Petrus J.A. and Kamber B.S. (2012)

VizualAge: A novel approach to laser ablation ICP-MS U-Pb geochronology data reduction. Geostandards and Geoanalytical Research, 36, 247-270.

Pichon L., Moignard B., Lemasson Q., Pacheco C. and Walter P. (2014)

Development of a multi-detector and a systematic imaging system on the AGLAE external beam. Nuclear Instruments and Methods in Physics Research B, 318, 27-31.

Pleßow A. (2013)

$\mathrm{X}$-ray-induced alteration of specimens as crucial obstacle in XRF spectrometry of fluorine in rocks and soils. X-Ray Spectrometry, 42, 19-32.

Prior D.J., Boyle A.P., Brenker F., Cheadle M.C., Austin D., Lopez G., Peruzzo L., Potts G.J., Reddy S., Spiess R., Timms N.E., Trimby P.W., Wheeler J. and Zetterström L. (1999)

The application of electron backscatter diffraction and orientation contrast imaging in the SEM to textural problems in rocks. American Mineralogist, 84, 1741-1759

Prior D.J., Diebold S., Obbard R., Daghlian C., Goldsby D.L., Durham W.B. and Baker I. (2012)

Insight into the phase transformations between Ice Ih and Ice II from electron backscatter diffraction data. Scripta Materialia, 66, 69-72.

Prior D.J., Lilly K., Seidmann M., Vaughan M., Becroft L., Easingwood R., Diedold S., Obbard R., Daghlian C., Backer I., Caswell T., Golding N., Goldsby D., Durham W.B., Piazolo S. and Wilson C.J.L. (2014) 
Making EBSD on water ice routine. Journal of Microscopy, (submitted).

Ramsey M.H. and Boon K.A. (2012)

Can in situ geochemical measurements be more fit-for-purpose than those made ex situ.

Applied Geochemistry, 27, 969-976.

Rehkämper M., Schönbächler M. and Stirling C.H. (2001)

Multiple collector ICP-MS: Introduction to instrumentation, measurement techniques and analytical capabilities. Geostandards Newsletter: The Journal of Geostandards and Geoanalysis, 25, 23-40.

Reichart P. and Dollinger G. (2009)

Hydrogen analysis by proton-proton scattering. In: Wang Y. and Nastasi M. (eds), Handbook of modern ion beam materials analysis (2nd edition), Materials Research Society (Warrendale, Pennyslvania, USA), 187-206.

Rybczynski N., Gosse J.C., Harington C.R., Wogelius R.A., Hidy A.J. and Buckley M. (2013)

Mid-Pliocene warm-period deposits in the high Arctic yield insight into camel evolution.

Nature Communications, 4, article 1550.

Reis M.A., Chaves P.C., Taborda A., Marques J.P. and Barradas N.P. (2014)

Fixed and free line ratio DT2 PIXE fitting and simulation package. Nuclear Instruments and Methods in Physics Research B, 318, 65-69.

Rittner M. and Müller W. (2012)

2D mapping of LA-ICP-MS trace element distributions using R. Computers and

Geosciences, 42, 152-161.

Roberts M.L. and Caffee M.W. (2000)

${ }^{129}$ I interlaboratory comparison: Phase II results. Nuclear Instruments and Methods in Physics Research B, 172, 388-394.

Roberts M.L., Caffee M.W. and Proctor I.D. (1997)

${ }^{129}$ I interlaboratory comparison. Nuclear Instruments and Methods in Physics Research B, 123, 367-370.

This article is protected by copyright. All rights reserved. 
Ros L., Borysiuk M., Kristiansson P., Abdel N., Elfman M., Golubev P., Nilsson E.J.C. and Pallon J. (2013)

Measurement of hydrogen isotopes, D/H-ratio in thin samples. Nuclear Instruments and Methods in Physics Research B, 306, 54-58.

Ros L., Borysiuk M., Kristiansson P., Abdel N., Elfman M., Nilsson E.J.C. and Pallon J. (2014)

Calibration of an elastic recoil setup for D/H-ratios close to natural abundance. Nuclear Instruments and Methods in Physics Research B, 332, 187-190.

Ross P.-S., Bourke A. and Fresia B. (2013)

A multi-sensor logger for rock cores: Methodology and preliminary results from the Matagami mining camp, Canada. Ore Geology Reviews, 53, 93-111.

Ross P.-S., Bourke A. and Fresia B. (2014a)

Improving lithological discrimination in exploration drill-cores using portable X-ray fluorescence measurements: (1) testing three Olympus Innov-X analysers on unprepared cores. Geochemistry: Exploration, Environment, Analysis, 14, 171-185.

Ross P.-S., Bourke A. and Fresia B. (2014b)

Improving lithological discrimination in exploration drill-cores using portable $\mathrm{X}$-ray fluorescence measurements: (2) applications to the $\mathrm{Zn}-\mathrm{Cu}$ Matagami mining camp, Canada. Geochemistry: Exploration, Environment, Analysis, 14, 187-196.

Roux C., Le Gal La Salle C., Simonucci C., Van Meir N., Fifield L.K., ASTER Team, Diez O., Bassot S., Simler R., Bugai D., Kashparov V. and Lancelot J. (2014) High ${ }^{36} \mathrm{Cl} / \mathrm{Cl}$ ratios in Chernobyl groundwater. Journal of Environmental Radioactivity, 138, 19-32.

Ryan C.G., Siddons D.P., Kirkham R., Li Z.Y., de Jonge M.D., Paterson D., Cleverley J.S., Kuczewski A., Dunn P.A., Jensen M., De Geronimo G., Howard D.L., Godel B., Dyl K.A., Fisher L.A., Hough R.H., Barnes S.J., Bland P.A., Moorhead G., James S.A., Spiers K.M., Falkenberg G., Boesenberg U. and Wellenreuther G. (2013)

The Maia detector array and X-ray fluorescence imaging system: locating rare precious metal phases in complex samples. In: Lai B. (ed.), X-ray nanoimaging: Instruments and Methods. SPIE Proceedings 8851, 88510Q.

This article is protected by copyright. All rights reserved. 
Sakaguchi A., Kadokura A., Steier P., Takahashi Y., Shizuma K., Hoshi M., Nakakuki T. and Yamamoto M. (2012)

Uranium-236 as a new oceanic tracer: A first depth profile in the Japan Sea and comparison with caesium-137. Earth and Planetary Science Letters, 333-334, 165-170.

Schimmelpfennig I., Schaefer J.M., Akçar N., Koffman T., Ivy-Ochs S., Schwartz R., Finkel R. C., Zimmerman S. and Schlüchter C. (2014)

A chronology of Holocene and Little Ice Age glacier culminations of the Steingletscher, Central Alps, Switzerland, based on high-sensitivity beryllium-10 moraine dating. Earth and Planetary Science Letters, 393, 220-230.

Schwehr K., Otosaka S., Merchel S., Kaplan D.I., Zhang S., Xu C., Li H.-P., Ho Y.-F., Yeager C.M., ASTER-Team and Santschi P.H. (2014)

Speciation of iodine isotopes inside and outside of a contaminant plume at the Savannah River Site. Science of the Total Environment, 497-498, 671-678.

Shaheen M.E., Gagnon J.E. and Fryer B.J. (2012)

Femtosecond (fs) lasers coupled with modern ICP-MS instruments provide new and improved potential for in situ elemental and isotopic analyses in the geosciences. Chemical Geology, 330-331, 260-273.

Shinonaga T., Steier P., Lagos M. and Ohkura T. (2014)

Airborne plutonium and non-natural uranium from the Fukushima DNPP Found at $120 \mathrm{~km}$ distance a few days after reactor hydrogen explosions. Environmental Science and Technology, 48, 3808-3814.

Siddons D.P., Kirkham R., Ryan C.G., De Geronimo G., Dragone A., Kuczewski A. J., Li Z.Y., Carini G.A., Pinelli D., Beuttenmuller R., Elliott D., Pfeffer M., Tyson T.A., Moorhead G.F. and Dunn P.A. (2014)

Maia X-ray microprobe detector array system. Journal of Physics: Conference Series, 499, 012001 .

Smolek S., Nakazawa T., Tabe A., Nakano K., Tsuji K., Streli C. and Wobrauschek P. (2014)

Comparison of two confocal micro-XRF spectrometers with different design aspects. X-Ray Spectrometry, 43, 93-101.

Smolek S., Pemmer B., Fölser M., Streli C. and Wobrauschek P. (2012)

This article is protected by copyright. All rights reserved. 
Confocal micro-X-ray fluorescence spectrometer for light element analysis. Review of Scientific Instruments, 83, 083703.

Souders A.K. and Sylvester P.J. (2008)

Improved in situ measurements of lead isotopes in silicate glasses by LA-MC-ICPMS using multiple ion counters. Journal of Analytical Atomic Spectrometry, 23, 535-543.

Steinhauser G. (2014)

Fukushima's forgotten radionuclides: A review of the understudied radioactive emissions.

Environmental Science and Technology, 48, 4649-4663.

Sylvester P.J. (ed.) (2008)

Laser ablation ICP-MS in the Earth Sciences: Current practices and outstanding issues.

Mineralogical Association of Canada Short Course, 40, 305-348.

Sylvester P. (2014)

Guest editorial: Laser-based analyses for geochemistry and geochronology. Geostandards and Geoanalytical Research, 38, 251-252.

Takei H., Yokoyama T., Makishima A. and Nakamura E. (2001)

Formation and suppression of $\mathrm{AlF}_{3}$ during $\mathrm{Hf}$ digestion of rock samples in Teflon bombs for precise trace element analyses by ICP-MS and ID-TIMS. Proceedings of the Japan Academy, Series B, 77, 13-17.

Tollstrup D.L., Xie L.W., Wimpenny J.B., Chin E., Lee C.T. and Yin, Q.-Z. (2012) A trio of laser ablation in concert with two ICP-MSs: Simultaneous, pulse-by-pulse determination of $\mathrm{U}-\mathrm{Pb}$ discordant ages and a single spot $\mathrm{Hf}$ isotope ratio analysis in complex zircons from petrographic thin sections. Geochemistry, Geophysics, Geosystems, 13, Q03017, doi:10.1029/2011GC004027.

Trimby P.W. (2012)

Orientation mapping of nanostructured materials using transmission Kikuchi diffraction in the scanning electron microscope. Ultramicroscopy, 120, 16-24.

Trimby P.W. and Cairney J.M. (2014)

Transmission Kikuchi diffraction in the scanning electron microscope: orientation mapping on the nanoscale. Advanced Materials and Processes, 172, 13-15.

This article is protected by copyright. All rights reserved. 
Ulianov A., Müntener O., Schaltegger U. and Bussy F. (2012)

The data treatment dependent variability of $\mathrm{U}-\mathrm{Pb}$ zircon ages obtained using mono-collector, sector field, laser ablation ICPMS. Journal of Analytical Atomic Spectrometry, 27, 663676.

Valley J.W., Cavosie A.J., Ushikubo1 T., Reinhard D.A., Lawrence D.F., Larson D.J., Clifton P.H., Kelly T.F., Wilde S.A., Moser D.E. and Spicuzza M.J. (2014)

Hadean age for a post-magma-ocean zircon confirmed by atom-probe tomography. Nature Geoscience, 7, 219-223.

Vallverdú J., Saladié P., Ross A., Huguet R., Cáceres I., Mosquera M., GarciaTabernero A., Estalrrich A., Lozano-Fernández I., Pineda-Alcalá A., Carrancho A., Villalaín J.J., Bourlès D., Braucher R., Lebatard A., Vilalta J., Esteban-Nadal M., Lluc Bennàsar M., Bastir M., López-Polín L., Ollé A., Maria Vergés J., Ros-Montoya S., Martínez-Navarro B., García A., Martinell J., Expósito I., Burjachs F., Agustí J. and Carbonel E. (2014)

Age and date for early arrival of the Acheulian in Europe (Barranc de la Boella, la Canonja, Spain), PLOS One, 9, e103634.

Van Malderen S.J.M., van Elteren J.T. and Vanhaecke F. (2015)

Development of a fast laser ablation-inductively coupled plasma-mass spectrometry cell for sub- $\mu \mathrm{m}$ scanning of layered materials. Journal of Analytical Atomic Spectrometry, DOI: 10.1039/C4JA00137K.

Vermeesch P. (2012)

On the visualisation of detrital age distributions. Chemical Geology, 312-313, 190-194.

Wacker L., Münsterer C., Hattendorf B., Christl M., Günther D. and Synal H.-A. (2013)

Direct coupling of a laser ablation cell to an AMS. Nuclear Instruments and Methods in Physics Research B, 294, 287-290.

Wang H.A., Grolimund D., Giesen C., Borca C.N., Shaw-Stewart J.R., Bodenmiller B. and Günther D. (2013)

Fast chemical imaging at high spatial resolution by laser ablation inductively coupled plasmamass spectrometry. Analytical Chemistry, 85, 10107-10116.

Weikusat I., De Winter D.A.M., Pennock G.M., Hayless M., Schneijdenberg C.T.W.M. 
and Drury M.R. (2010)

Cryogenic EBSD on ice: Preserving a stable surface in a low pressure SEM. Journal of Microscopy, doi: 10.1111/j.1365-2818.2010.03471.x

West M., Ellis A.T., Potts P.J., Streli C., Vanhoof C. and Wobrauschek P. (2014)

2014 atomic spectrometry update - A review of advances in X-ray fluorescence spectrometry Journal of Analytical Atomic Spectrometry, 29, 1516-1563.

Whitehouse M. (2013)

Multiple sulfur isotope determination by SIMS: Evaluation of reference sulfides for $\Delta^{33} \mathrm{~S}$ with observations and a case study on the determination of $\Delta^{36} \mathrm{~S}$. Geostandards and

Geoanalytical Research, 37, 19-33.

Wiedenbeck M. (2011)

Seeing single atoms. Elements, 7, 79-80.

Wiedenbeck M., Bugoi R., Duke J.J.M, Dunai T., Enzweiler J., Horan M., Jochum K.P., Linge K., Košler J., Merchel S., Morales L.F.G., Nasdala L., Stalder R., Sylvester P., Weis U. and Zoubir A. (2012)

GGR biennial critical review: Analytical developments since 2010. Geostandards and Geoanalytical Research, 36, 337-398.

Wilcken K.M., Freeman S.P.H.T., Schnabel C., Binnie S.A., Xua S. and Phillips R.J. (2013)

${ }^{36} \mathrm{Cl}$ accelerator mass spectrometry with a bespoke instrument. Nuclear Instruments and Methods in Physics Research B, 294, 107-114.

Winkler S.R., Steier P. and Carilli J. (2012)

Bomb fall-out ${ }^{236} \mathrm{U}$ as a global oceanic tracer using an annually resolved coral core. Earth and Planetary Science Letters, 359-360, 124-130.

Wirth R. (2009)

Focused Ion Beam combined with SEM and TEM: Advanced analytical tools for studies of chemical composition, microstructure and crystal structure in geomaterials at nanoscale.

Chemical Geology, 261, 217-229.

Wirth R. and Morales L. (2012)

Combined FIB-SEM-TEM techniques: Advanced tools to resolve microstructures

This article is protected by copyright. All rights reserved. 
and minerals phases in sedimentary rocks. Mineralogical Association of Canada Short Course, 42 (St. John's NL), 17-34.

Woodhead J., Hellstrom, J., Hergt J., Greig A. and Maas, R. (2007)

Isotopic and elemental imaging of geological materials by laser ablation inductively coupled plasma-mass spectrometry. Geostandards and Geoanalytical Research, 31, 331-343.

Wright S.I. and Adams B.L. (1992)

Automatic analysis of electron backscatter diffraction patterns. Metallurgical Transactions, 23A, 759-767.

Wunderlich R., Klingner N., Vogt J. and Spemann D. (2013)

Quantitative elemental microscopy on lateral highly inhomogeneous meteorite samples using ion beam analysis. Nuclear Instruments and Methods in Physics Research B, 306, 85-89.

Yang W., Lin A.-T., Zhang J.-C., Hao. J.-L., Shen W.-J. and Hu S. (2012)

Precise micrometre-sized $\mathrm{Pb}-\mathrm{Pb}$ and $\mathrm{U}-\mathrm{Pb}$ dating with NanoSIMS. Journal of Analytical and Atomic Spectrometry, 27, 479-487.

Young N.E., Briner J.P., Rood D.H. and Finkel R.C. (2012)

Glacier extent during the Younger Dryas and 8.2-ka Event on Baffin Island, Arctic Canada, Science, 337, 1330-1333.

Young N.E., Schaefer J.M., Briner J.P. and Goehring B.M. (2013)

A ${ }^{10}$ Be production-rate calibration for the Arctic. Journal of Quaternary Science, 28, 515526.

Zerathe S., Braucher R., Lebourg T., Bourlès D., Manetti M. and Léanni L. (2013)

Dating chert (diagenetic silica) using in-situ produced ${ }^{10} \mathrm{Be}$ : Possible complications revealed through a comparison with ${ }^{36} \mathrm{Cl}$ applied to coexisting limestone. Quaternary

Geochronology, 17, 81-93.

Zhang L., Hou X., Zhou W., Chen N., Liu Q., Luo M., Fan Y. and Fu Y. (2013) Performance of Accelerator Mass Spectrometry for ${ }^{129} \mathrm{I}$ using $\mathrm{AgI}-\mathrm{AgCl}$ carrier-free coprecipitation. Nuclear Instruments and Methods in Physics Research B, 294, 276-280.

Zhang L., Ren Z.-Y., Nichols A.R.L., Zhang Y-H., Zhang Y., Qian S-P. and Liu J.-Q. (2014) 
Lead isotope analysis of melt inclusions by LA-MC-ICP-MS. Journal of Analytical Atomic Spectrometry, 29, 1393-1405.

Zhang W., Hu Z., Liu Y., Chen L., Chen H., Li M., Zhao L., Hu S. and Gao S. (2012) Reassessment of $\mathrm{Hf} / \mathrm{HNO}_{3}$ decomposition capability in the high-pressure digestion of felsic rocks for multi-element determination by ICP-MS. Geostandards and Geoanalytical Research, 36, 271-289.

\section{Zhou Q., Yin Q.-Z., Young E.D., Li X.-H. Wu F.-Yu, Li Q.-L, Liu Y. and Tang G.-Q.} (2013)

SIMS $\mathrm{Pb}-\mathrm{Pb}$ and $\mathrm{U}-\mathrm{Pb}$ age determination of eucrite zircons at $<5 \mu \mathrm{m}$ scale and the first 50 Ma of the thermal history of Vesta. Geochimica et Cosmochimica Acta, 110, 152-175.

Zucchiatti A. and Redondo-Cubero A. (2014)

Ion beam analysis: New trends and challenges. Nuclear Instruments and Methods in Physics Research B, 331, 48-54.

Zucchiatti A., Alonso U., Lemasson Q., Missana T., Moignard B., Pacheco C., Pichon L. and Camarena de la Mora S. (2014)

Detection of actinides and rare earths in natural matrices with the AGLAE new, high sensitivity detection set-up. Nuclear Instruments and Methods in Physics Research B, 332, 245-250.

\section{Figure Captions}

Figure 1. Schematic diagram of the MP-AES waveguide, with axial magnetic excitation and radial electric field.

Figure 2. Examples of XRF-based devices that have come onto the market in recent years: (A) microXRF (EDAX Eagle III) with computers. (B) Handheld XRF (Niton XL3t GOLDD+) with reference materials.

Figure 3. Schematic representation of a Faraday cup detection system. The Faraday cup is connected to electrical ground by an amplifier that is equipped with a high value resistor (commonly $10^{11}$ or $10^{12} \Omega$ ). The amplified signal in converted to volts by a voltage-tofrequency converter. Figure adapted from Koornneef et al. 2013.

This article is protected by copyright. All rights reserved. 
Figure 4. Top panel: example of FC/FC runs from a single session in which little or no trend in ${ }^{34} \mathrm{~S} /{ }^{32} \mathrm{~S}$ was observed over the course of minute-long pyrite analyses. Middle panel: example for a different analytical session in which a clear trend is seen in the measured ${ }^{34} \mathrm{~S} /{ }^{32} \mathrm{~S}$, note the differences in the slopes between the pyrite (Py) and pyrrhotite (Po) analyses. For both top and middle panels the Y-axis is\%o change from the first cycle in the individual run. Bottom panel: example of $\delta^{34} S$ vs. $\Delta^{36} S$ for a variety of Archaean pyrite samples investigated by Whitehouse (2013), showing that the dispersion in the data for both systems well exceeds the analytical uncertainty. Crosses indicate $2 s$ uncertainty estimates. $\mathrm{MDF}=$ mass dependent fractionation line. All panels courtesy of M. Whitehouse.

Figure 5. (A) Internal view of the vacuum chamber of the Dual Beam machine ${ }^{\circledR}$, installed at the GFZ-Potsdam, which is typical for such instrumentation. (B) 3D reconstruction showing pore space, quartz, calcite and organic matter of a sample of Opalinus Clay analysed via FIB nanotomography. Image courtesy L. Keller (Zürich University of Applied Sciences, Switzerland), also published in Keller et al. (2013).

Figure 6. (A) General view of the cryo-SEM-EBSD system Zeiss Sigma VP FEG, including the nitrogen glove box, operating at the Otago Centre of Electron Microscopy (University of Otago, New Zealand). (B) EBSD orientation map on experimentally deformed standard ice, axial load was vertical and parallel to [0001] in the inverse pole figure colour code. Images A and B courtesy of Prof. D. Prior at University of Otago, New Zealand. (C) Schematic illustration a SEM chamber illustrating the setup for the Transmission Kikuchi Diffraction method using a FIB-prepared TEM foil. Image courtesy of P. Trimby, extracted from Trimby and Cairney (2014). (D) Transmission Kikuchi Diffraction orientation map of a diamond crystal (blue) with an inclusion of pyrrhotite (pink) and a rim of magnetite nanocrystals. Crystallographic orientation map using a step size of $10 \mathrm{~nm}$. Sample courtesy of D. Jacob (Macquarie University), TKD map courtesy P. Trimby, D. Jacob and S. Piazolo at the Australian Centre for Microscopy and Microanalysis at the University of Sydney.

Figure 7. Top: Overview of the LEAP $5000 \mathrm{HR}^{\mathrm{TM}}$, latest in the line of commercially available APT instruments from Cameca. Middle: Close up view of a sample array on the left side and the Local Electrode ${ }^{\mathrm{TM}}$ for extracting evaporated ions and accelerating them into the APT time of flight spectrometer. Bottom: High resolution SEM image of a sample tip prior to APT analysis - in this particular case NIST SRM 2135c Ni/Cr thin film depth profile standard. All three images courtesy of Cameca.

Figure 8. Example of APT determined element distributions in a $4.4 \mathrm{Ga}$ zircon. Left section shows the distributions of lead and yttrium as recorded from the entire $\sim 1 \mu \mathrm{m}$ long sample.

This article is protected by copyright. All rights reserved. 
The middle section shows a $100 \mathrm{~nm}$ x $100 \mathrm{~nm}$ detail from the same data suite. The right side of the figure is a detail from a single Y-rich cluster (image courtesy J. Valley, see also Valley et al. 2014).

Table 1.

Summary of main XRF instruments for geoanalysis

\begin{tabular}{|c|c|c|c|c|}
\hline Instrument & Acronym & Portable & Detector & Sample type \\
\hline $\begin{array}{l}\text { Laboratory X- } \\
\text { ray } \\
\text { fluorescence }\end{array}$ & XRF & No & WDS or EDS & $\begin{array}{l}\text { Glass disks, } \\
\text { pressed pellets } \\
\text { or liquids }\end{array}$ \\
\hline $\begin{array}{l}\text { Micro X-ray } \\
\text { fluorescence } \\
\text { (Figure 2A) }\end{array}$ & Micro-XRF & No & EDS & $\begin{array}{l}\text { Glass disks, } \\
\text { pressed pellets, } \\
\text { polished thin- } \\
\text { sections and } \\
\text { unprepared } \\
\text { samples }\end{array}$ \\
\hline $\begin{array}{l}\text { Handheld X- } \\
\text { ray } \\
\text { fluorescence } \\
\text { (Figure 2B) }\end{array}$ & HHXRF & Yes & EDS & $\begin{array}{l}\text { Glass disks, } \\
\text { pressed pellets } \\
\text { and unprepared } \\
\text { samples }\end{array}$ \\
\hline $\begin{array}{l}\text { Portable X-ray } \\
\text { fluorescence }\end{array}$ & PXRF & Yes & EDS & $\begin{array}{l}\text { Glass disks, } \\
\text { pressed pellets } \\
\text { and unprepared } \\
\text { samples }\end{array}$ \\
\hline $\begin{array}{l}\text { X-ray } \\
\text { fluorescence } \\
\text { core scanner }\end{array}$ & & No & EDS & $\begin{array}{l}\text { Split core of } \\
\text { rock or } \\
\text { unconsolidated } \\
\text { sediments }\end{array}$ \\
\hline $\begin{array}{l}\text { Scanning } \\
\text { electron } \\
\text { microscope } \\
\text { attachment }\end{array}$ & SEM & No & EDS or WDS & $\begin{array}{l}\text { Polished thin } \\
\text { sections, grain } \\
\text { mounts, }\end{array}$ \\
\hline $\begin{array}{l}\text { Electron probe } \\
\text { micro-analysis }\end{array}$ & EPMA & No & WDS or EDS & $\begin{array}{l}\text { Polished thin } \\
\text { sections or } \\
\text { grain mounts }\end{array}$ \\
\hline
\end{tabular}

This article is protected by copyright. All rights reserved. 

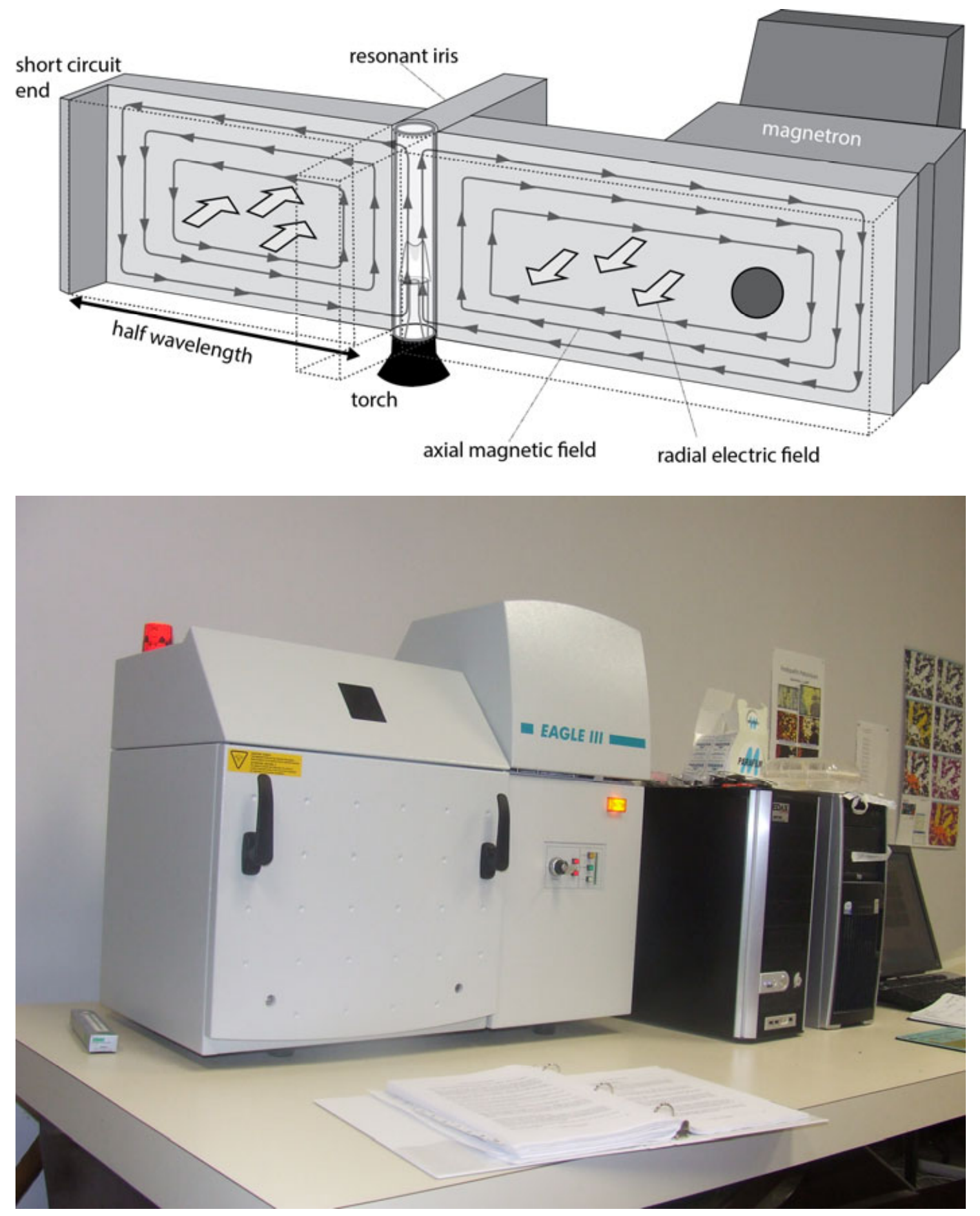

This article is protected by copyright. All rights reserved. 

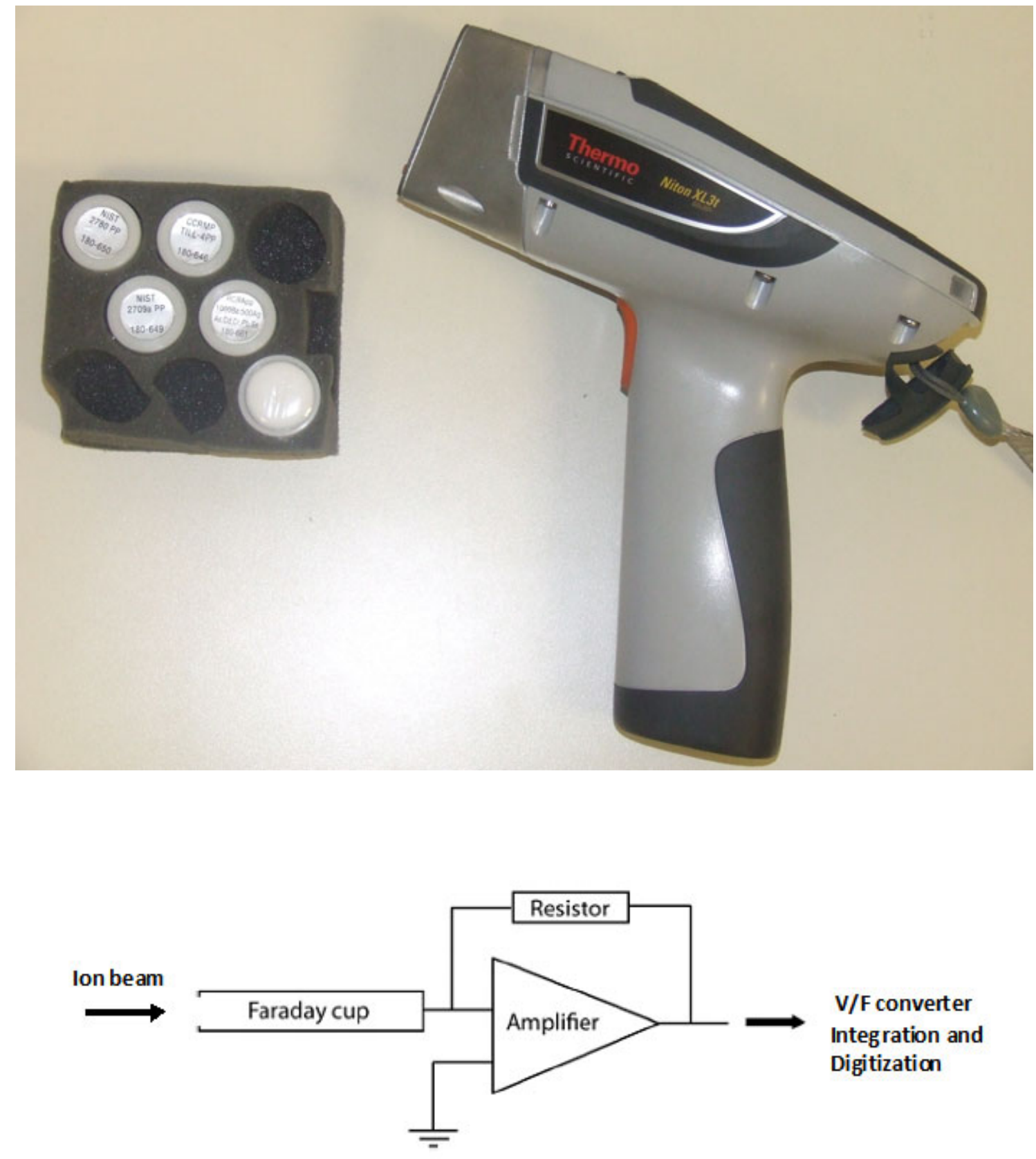

This article is protected by copyright. All rights reserved. 

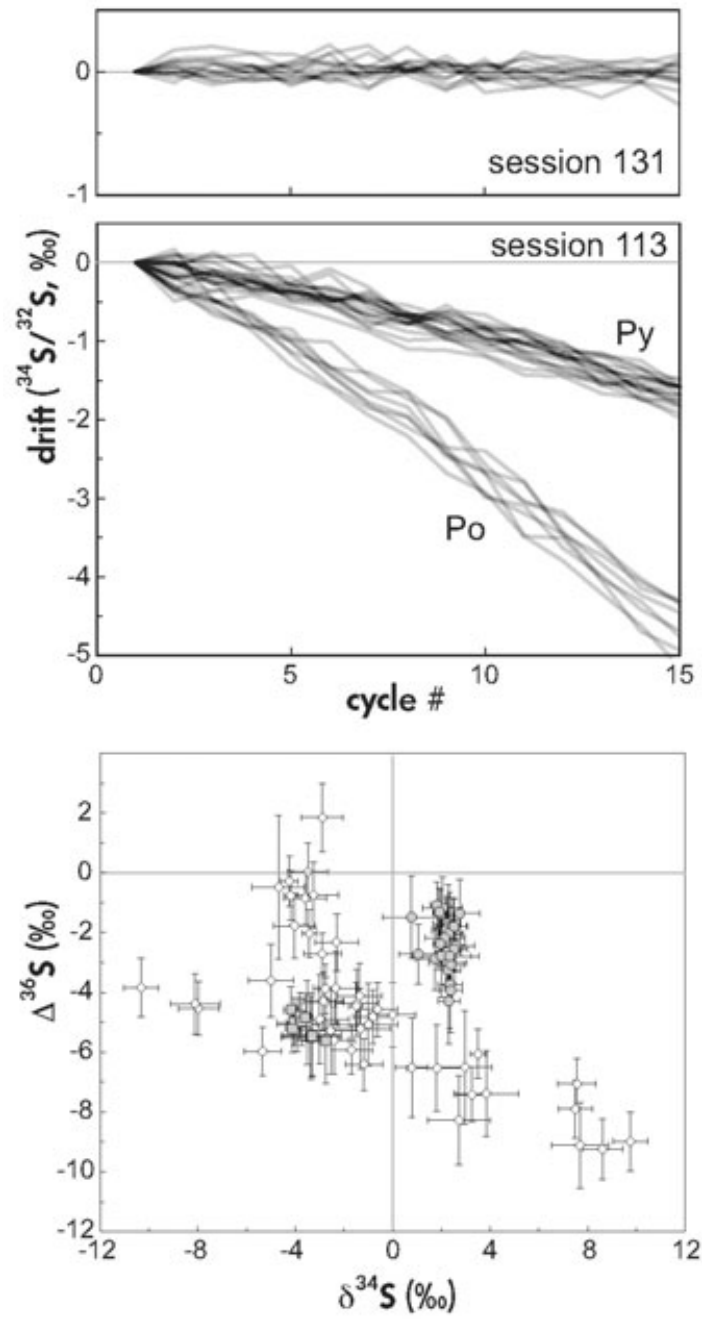

This article is protected by copyright. All rights reserved. 

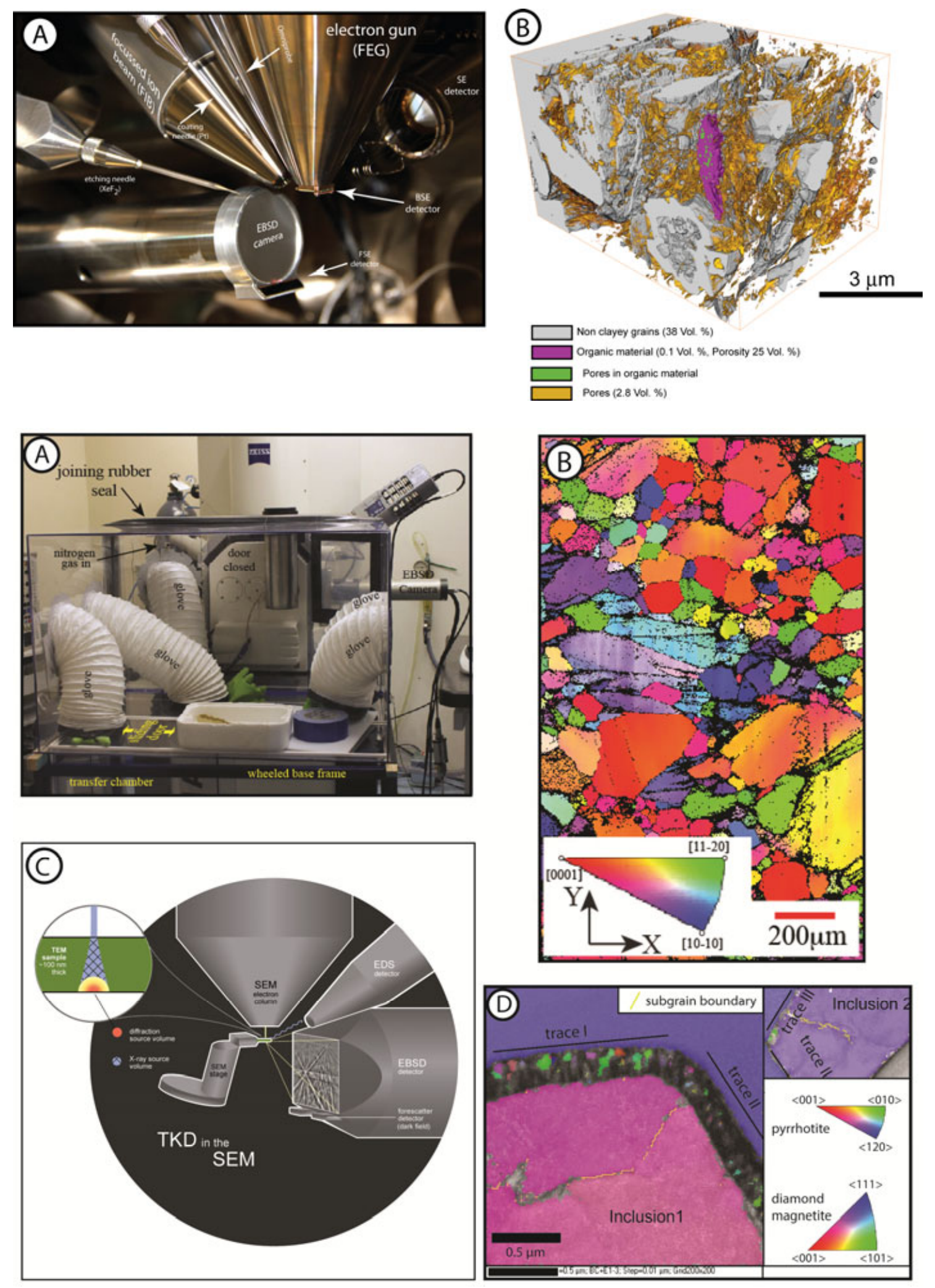

This article is protected by copyright. All rights reserved. 

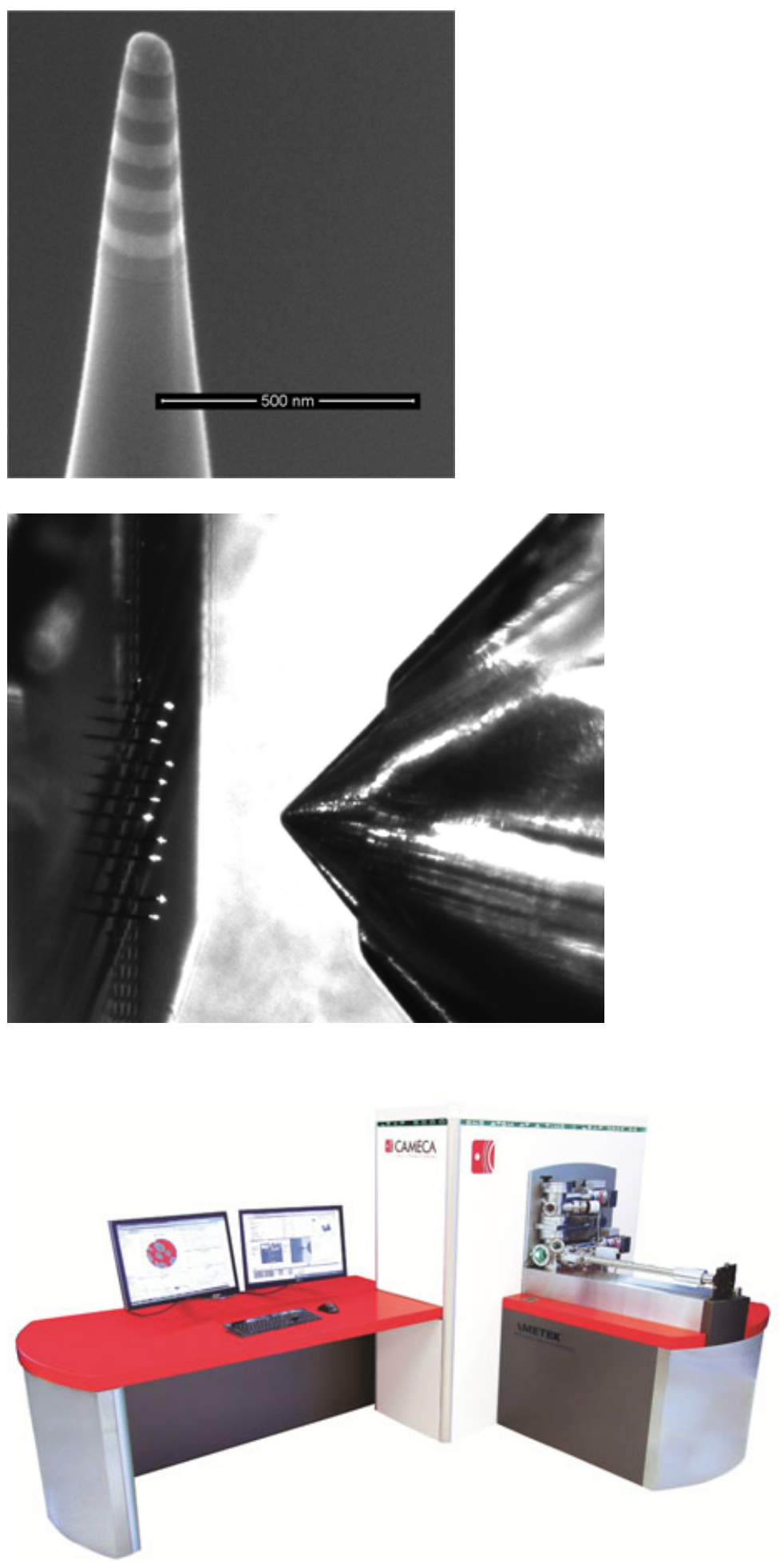

This article is protected by copyright. All rights reserved. 


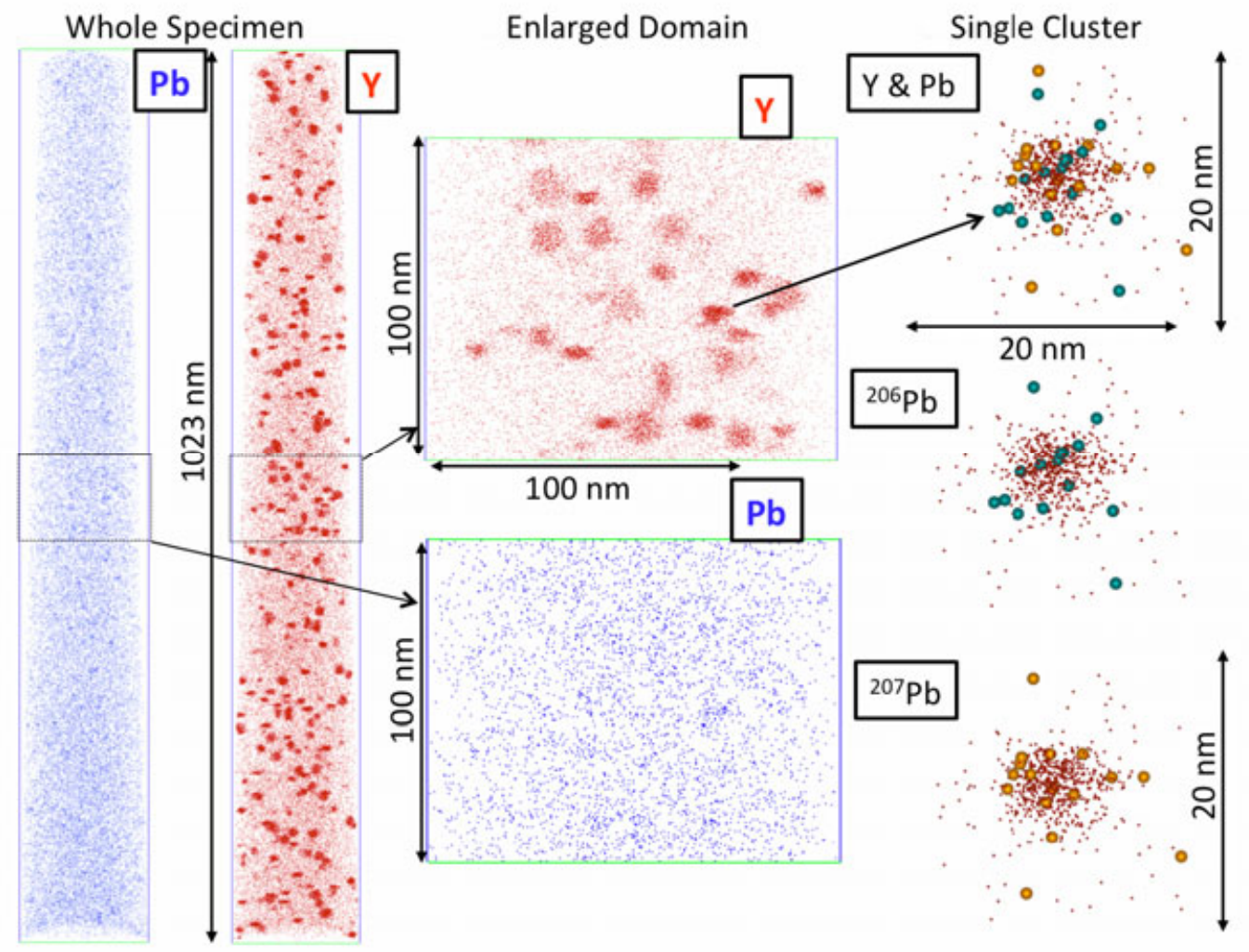

This article is protected by copyright. All rights reserved. 